University of Tennessee Health Science Center

UTHSC Digital Commons

\title{
Neocortical Layer 4 to Layer 2/3 Sensory Information Processing Investigated with Digital-Light-Projection Neuronal Photostimulation
}

Jason Paul Jerome

University of Tennessee Health Science Center

Follow this and additional works at: https://dc.uthsc.edu/dissertations

Part of the Neurosciences Commons

\section{Recommended Citation}

Jerome, Jason Paul , "Neocortical Layer 4 to Layer 2/3 Sensory Information Processing Investigated with Digital-Light-Projection Neuronal Photostimulation" (2012). Theses and Dissertations (ETD). Paper 138. http://dx.doi.org/10.21007/etd.cghs.2012.0153. 


\title{
Neocortical Layer 4 to Layer 2/3 Sensory Information Processing Investigated with Digital-Light-Projection Neuronal Photostimulation
}

\author{
Abstract \\ The mammalian brain forms neuronal networks and microcircuits with cell-type- and anatomical-specific \\ synaptic connections. Despite great advances in elucidating the cellular physiology of the nervous \\ system, little is known about the computational processes occurring at the level of neuronal \\ microcircuits. Much success has been reported in describing the synaptic input patterns of many brain \\ regions and cell types using photostimulation systems; however, these systems are severely limited in \\ their ability to study the integration of synaptic input from multiple synchronous or temporally correlated \\ presynaptic locations. Here we describe a system that allows the generation of arbitrary 2-D stimulus \\ patterns with thousands of independently controlled sites to manipulate the activity of populations of \\ neurons with high spatial and temporal precision. The PC-controlled Digital-Light-Processing (DLP) based \\ system updates the 780,000 parallel photostimulation beams, or pixels, at a maximum rate of $13 \mathrm{kHz}$. \\ With the currently used projection objective, the pixel sizes at the plane of focus are $7.3 \mu \mathrm{m} 2$. The high- \\ power UV laser source used in this system provides a light flux density sufficient for bins of $8 \times 8$ pixels \\ $(21.6 \mu \mathrm{m} \times 21.6 \mu \mathrm{m})$ with dwell times as low $3 \mathrm{~ms}$ to reliably induce action potentials in $2.5 \mathrm{mM} \mathrm{MNI}-$ \\ caged glutamate. At these settings the effective diameter of a glutamate uncaging site is $<86 \mu \mathrm{m}$, which \\ is equivalent to most other UV photostimulation rigs. With DLP photostimulation, sub-threshold \\ responses and action potentials can be synchronously induced at thousands of sites over a $2.76 \mathrm{~mm} x$ \\ $2.07 \mathrm{~mm}$ area, a capability unmatched by any other current system. This DLP-based system has the \\ unique capability to investigate normal and diseased circuit properties by investigating neuronal \\ responses to spatiotemporally complex activity patterns. This technique was used to investigate the \\ temporal integration of synaptic input in the whisker barrel cortex of mice. The neocortex is organized \\ into layers, with neuronal networks and circuits formed by layer-specific connections. While the \\ anatomical organization of these circuits has been well characterized, the information processing and \\ coding performed by these ensembles is poorly understood. A key component of this investigation \\ concerns the transmission and transformation of the neuronal representation from one neuronal pool to \\ the next. In the rodent somatosensory barrel cortex, histologically-distinguishable "barrels" in layer 4 (L4) \\ receive principal input from a single whisker. L4 projects to layer II/III (L2/3), where the circuit diverges to \\ multiple postsynaptic targets. Using the DLP-photostimulation system, we modulated the synchronicity of \\ action potentials in L4 cells while recording from L2/3 in an acute slice preparation. This data shows that \\ synchronous activity in L4 neurons is highly effective at eliciting strong spiking responses in L2/3 \\ pyramidal cells, while asynchronous $L 4$ activity fails to drive $L 2 / 3$ to action-potential threshold. \\ Pharmacological manipulation of the slice-bathing solution has suggested that this phenomenon is \\ AMPA-receptor dependent and modulated by NMDA receptor activity. Intracellular pharmacological \\ manipulations suggest that postsynaptic conductances also play a role in the nonlinear L2/3 synaptic \\ integration of $L 4$ activity.

\section{Document Type} \\ Dissertation \\ Degree Name \\ Doctor of Philosophy (PhD) \\ Program \\ Biomedical Sciences
}




\section{Research Advisor}

Detlef Heck Ph.D.

\section{Keywords}

barrel cortex, neocortex, neuronal microcircuit, neurophysiology, photostimulation, synaptic connectivity

\section{Subject Categories}

Medical Sciences | Medicine and Health Sciences | Neurosciences

\section{Comments}

Two year embargo expired May 2014 
Neocortical Layer 4 to Layer 2/3 Sensory Information Processing Investigated with Digital-Light-Projection Neuronal Photostimulation

\author{
A Dissertation \\ Presented for \\ The Graduate Studies Council \\ The University of Tennessee \\ Health Science Center
}

\author{
In Partial Fulfillment \\ Of the Requirements for the Degree \\ Doctor of Philosophy \\ From The University of Tennessee
}

By

Jason Paul Jerome

May 2012 
Copyright (C) 2012 by Jason Jerome. All rights reserved. 


\section{DEDICATION}

This dissertation is dedicated to my loving wife, Mrs. Lacy Jerome, whose patience and limitless devotion allows me to approach every challenge with confidence and determination. 


\section{ACKNOWLEDGEMENTS}

I would like to thank my advisor, Dr. Detlef Heck, for the continuing personal and professional motivation he provides. Without his support, this work would not have been possible. I would also like to thank my committee members, Dr. William Armstrong, Dr. Robert Foehring, Dr. Jay Callaway, and Dr. Chrysanthe Preza whose guidance, support, and useful suggestions have maximized the scientific soundness of the work presented here.

I would also like to recognize my mother, whose love, support, and encouragement has pushed me through many of life's most difficult challenges. My brother, Justin, who knows me better than anyone, has provided balance and continuity. My father, Chris Jerome, who has been a model for work ethic and character as well as Madeleine and Margot for their part in building the foundation on which I've achieved my educational goals.

I would also like to thank Dr. Mitch Watsky and Dr. Mel Park and the other UTHSC RyuTe students with whose teaching and training has instilled a sense of confidence and security that I will carry with me for the rest of my life.

Finally, I would like to thank my loving and devoted wife Lacy, for whom I will be eternally grateful. Fulfilling my educational goals is and will be a long and arduous road, but with Lacy's devotion and support, the light at the end of the tunnel is blindingly bright. 


\begin{abstract}
The mammalian brain forms neuronal networks and microcircuits with cell-typeand anatomical-specific synaptic connections. Despite great advances in elucidating the cellular physiology of the nervous system, little is known about the computational processes occurring at the level of neuronal microcircuits. Much success has been reported in describing the synaptic input patterns of many brain regions and cell types using photostimulation systems; however, these systems are severely limited in their ability to study the integration of synaptic input from multiple synchronous or temporally correlated presynaptic locations.
\end{abstract}

Here we describe a system that allows the generation of arbitrary 2-D stimulus patterns with thousands of independently controlled sites to manipulate the activity of populations of neurons with high spatial and temporal precision. The PC-controlled Digital-Light-Processing (DLP) based system updates the 780,000 parallel photostimulation beams, or pixels, at a maximum rate of $13 \mathrm{kHz}$. With the currently used projection objective, the pixel sizes at the plane of focus are $7.3 \mu \mathrm{m}^{2}$. The high-power UV laser source used in this system provides a light flux density sufficient for bins of $8 \times 8$ pixels $(21.6 \mu \mathrm{m} \times 21.6 \mu \mathrm{m})$ with dwell times as low $3 \mathrm{~ms}$ to reliably induce action potentials in $2.5 \mathrm{mM}$ MNI-caged glutamate. At these settings the effective diameter of a glutamate uncaging site is $<86 \mu \mathrm{m}$, which is equivalent to most other UV photostimulation rigs. With DLP photostimulation, sub-threshold responses and action potentials can be synchronously induced at thousands of sites over a $2.76 \mathrm{~mm} \times 2.07 \mathrm{~mm}$ area, a capability unmatched by any other current system. This DLP-based system has the unique capability to investigate normal and diseased circuit properties by investigating neuronal responses to spatiotemporally complex activity patterns.

This technique was used to investigate the temporal integration of synaptic input in the whisker barrel cortex of mice. The neocortex is organized into layers, with neuronal networks and circuits formed by layer-specific connections. While the anatomical organization of these circuits has been well characterized, the information processing and coding performed by these ensembles is poorly understood. A key component of this investigation concerns the transmission and transformation of the neuronal representation from one neuronal pool to the next. In the rodent somatosensory barrel cortex, histologically-distinguishable "barrels" in layer 4 (L4) receive principal input from a single whisker. L4 projects to layer II/III (L2/3), where the circuit diverges to multiple postsynaptic targets. Using the DLP-photostimulation system, we modulated the synchronicity of action potentials in L4 cells while recording from L2/3 in an acute slice preparation. This data shows that synchronous activity in L4 neurons is highly effective at eliciting strong spiking responses in L2/3 pyramidal cells, while asynchronous L4 activity fails to drive L2/3 to action-potential threshold. Pharmacological manipulation of the slice-bathing solution has suggested that this phenomenon is AMPA-receptor dependent and modulated by NMDA receptor activity. Intracellular pharmacological manipulations suggest that postsynaptic conductances also play a role in the nonlinear L2/3 synaptic integration of L4 activity. 


\section{TABLE OF CONTENTS}

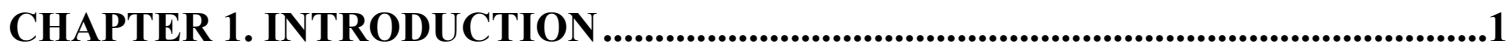

Specific Aim \# 1 - Development of a DLP Neuronal Photostimulation System .............1 Specific Aim \# 2 - Neocortical Temporal Synaptic Integration of L4 Input to L2/3......1

CHAPTER 2. SIGNIFICANCE _.................................................................................

Neocortical L4 to L2/3 Temporal Integration................................................................

Photostimulation Techniques...............................................................................

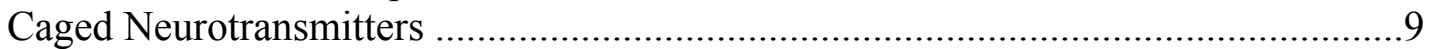

Uncaging with Ultraviolet Light................................................................. 10

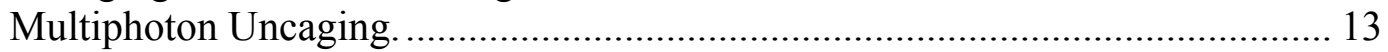

Parallel Photostimulation Techniques..................................................................15

CHAPTER 3. MATERIALS AND METHODS......................................................17

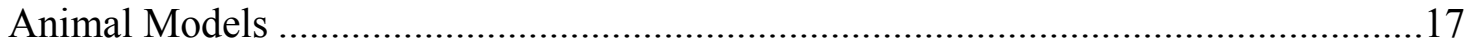

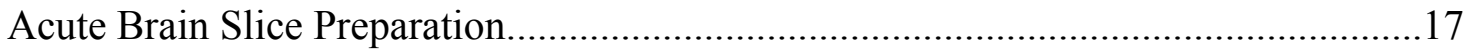

Whole-Cell Current Clamp Recordings from Acute Brain Slices .................................18

On-Cell Recordings from Acute Brain Slices...............................................................19

Digital Light Projection Electronics and Software ……...........................................19

Digital Light Projection Optical and Mechanical Design...........................................20

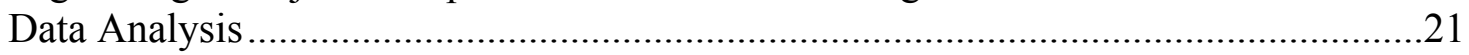

Calculation of Summation Ratio and Supralinearity ………………………….......21

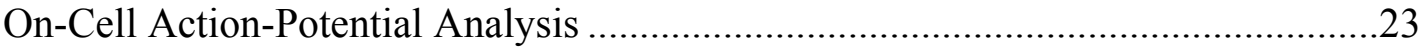

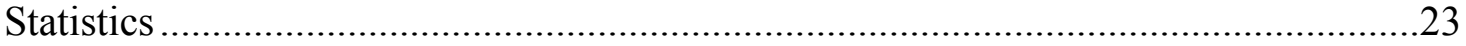

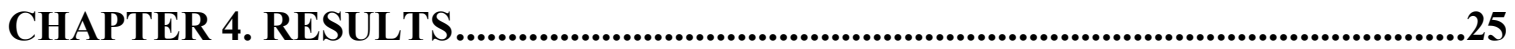

System Design and Optical Performance ……………........................................25

Determining Physiological Resolution of DLP Photostimulation ..................................29

Spatiotemporally Complex Direct-Dendritic Photostimulation ……............................30

Photostimulation-Induced L4 Excitatory Synaptic Input to L2/3 .................................33

Timing-Dependent Nonlinear Temporal Summation ....................................................38

L2/3 Postsynaptic Temporal Integration of L4 Synaptic Input .....................................42

L4 Presynaptic Mechanisms of Supralinear Synaptic Summation................................45

AMPA Dependence and NMDA Modulation of Temporal Synaptic Integration .........49

Anatomical Arrangement and Temporal Order of Stimulus Sites................................52

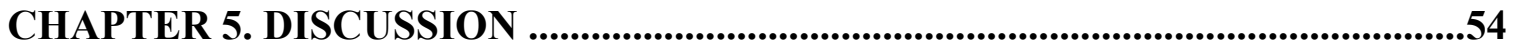

Interlaminar Cortical Processing of Sensory Input......................................................54

Digital Parallel Photostimulation...............................................................................5

LIST OF REFERENCES.............................................................................................59 


\section{APPENDIX. EXAMPLE CODE FOR CONTROLLING THE DLP}

PHOTOSTIMULATION SYSTEM..........................................................................71

VITA 


\section{LIST OF TABLES}

Table 4-1. Maximum and minimum estimates of the number of neurons presynaptic to L2/3 stimulated by activation of single L4 sites ....................................37 


\section{LIST OF FIGURES}

Figure 2-1. Input and intracortical pathways of the somatosensory barrel cortex............6

Figure 3-1. DLP illumination mirror and mechanical design ...................................22

Figure 4-1. Principal of DLP neuronal photostimulation .........................................26

Figure 4-2. Optical performance of the DLP photostimulation system and resolution of glutamate uncaging

Figure 4-3. Direct dendritic photostimulation of a neocortical layer V pyramidal neuron.

Figure 4-4. Presynaptic DLP photostimulation of neocortical L4 and the postsynaptic response in $\mathrm{L} 2 / 3$

Figure 4-5. Pre- and post-synaptic response to L4 photostimulation

Figure 4-6. Timing-dependence of postsynaptic action potential induction in response to parallel photostimulation.

Figure 4-7. Calculation of Summation Ratio (SR) and timing-dependence of synaptic temporal summation.

Figure 4-8. Intracellular pharmacological manipulations of L2/3 pyramidal cells and the resultant electrophysiological responses to L4 photostimulation

Figure 4-9. Quantification of L4 action-potential output ....

Figure 4-10. Example paired on-cell L4 recording in response to photostimulation with an ISI of $0 \mathrm{~ms}$

Figure 4-11. Extracellular pharmacological manipulations of network-wide glutamate-gated currents and the resultant L2/3 electrophysiological responses to L4 photostimulation. 


\section{LIST OF ABBREVIATIONS}

aCSF

AMPA

AP-5

ATP

CHR2

DLL

DLP

DMD

EGTA

EPSP

GABA

GUI

HEPES

$\mathrm{L} 2 / 3$

L4

MNI-Glu

NA

NBQX

NIR

NMDA

$\mathrm{PC}$

QX-314

RuBi-Glu

SLM

SR

TIRF

USB

UV

VPm

WM

$2 \mathrm{P}$ artificial CerbroSpinal Fluid

2-Amino-3-(5-Methyl-3-oxo-1,2- oxazol-4-yl) Propanoic Acid 2R-Amino-5-Phosphonopentanoate

Adenosine Triphosphate

Channelrhodopsin-2

Dynamic-Link Library

Digital Light Projection

Digital Micromirror Device

Ethylene Glycol Tetraacetic Acid

Excitatory PostSynaptic Potential

Gamma-AminoButyric Acid

Graphical User Interface

4-(2-HydroxyEthyl)-1-PiperazineEthaneSulfonic acid neocortical Layer II/III

neocortical Layer IV

4-Methoxy-7-NitroIndolinyl-caged-L-Glutamate

Numerical Aperature

2,3-dihydroxy-6-Nitro-7-sulfamoyl-Benzo[f]QuinoXaline-2,3dione

Near InFrared

N-Methyl-D-Aspartic Acid

Personal Computer (Microsoft Windows operating system)

$\mathrm{N}-(2,6$-dimethylphenylcarbamoylmethyl) triethylammonium chloride

RUthenium-BIpyridine-triphenylphosphine-caged-L-Glutamate Spatial Light Modulator

Summation Ratio

Total Internal ReFlection

Universal Serial Bus

UltraViolet electromagnetic radiation

Ventral posteromedial nucleus of the thalamus

White Matter

Two-Photon excitation fluorescence microscopy 


\section{CHAPTER 1. INTRODUCTION}

Despite great advances in the elucidation of the molecular mechanisms and cellular physiology of the nervous system, astoundingly little is known about the computational processes occurring at the level of neuronal microcircuits. A very significant but poorly understood aspect of this field is the contribution of single neurons to computations performed by complexly connected neural networks. The specific aims of this dissertation work were conceived with the goal of contributing to this large and heavily invested field of research. This introduction serves to briefly outline these specific aims. A detailed justification and background (literature review) of these specific aims can be found in Chapter 2.

\section{Specific Aim \# 1 - Development of a DLP Neuronal Photostimulation System}

Much success has been reported in describing the microcircuitry and synaptic input patterns of many brain regions and cell types using photostimulation systems, however, these systems are severely limited for the study of integration of synaptic input from multiple synchronous or temporally correlated presynaptic locations.

This first specific aim of this dissertation is intended to overcome these technical limitations by developing a versatile neuronal photostimulation system based on Digital Light Projection technology with the following unique capabilities: synchronous induction of action potentials in multiple targetable sites within a brain slice, large targetable photostimulation area, user-friendly interface for planning and executing user-define spatiotemporal photostimulation patterns, and relative ease and economy of integration into electrophysiology rigs.

\section{Specific Aim \# 2 - Neocortical Temporal Synaptic Integration of L4 Input to L2/3}

The second specific aim is to use the unique capabilities of this photostimulation system to investigate the temporal synaptic integration of L4 input to L2/3 in acute slices of the whisker barrel cortex of mice. Induction of action potentials in multiple L 4 neurons and simultaneous whole-cell recordings in L2/3 will allow us to investigate the temporal summation of synaptic inputs from L4 by L2/3 neurons. We hypothesized that synchronous stimulation of all ten sites will propagate much more effectively than desynchronized stimuli and that synchronized stimuli will summate supralinearly.

We pharmacologically manipulated the intracellular environment of L2/3 pyramidal cells with QX-314 and EGTA. The addition of QX-314 to the intracellular environment blocks several voltage-gate channels $\left(\mathrm{Na}^{+}, \mathrm{K}^{+}, \mathrm{Ca}^{2+}\right)$ from within the recorded cell. This manipulation was designed to test the hypothesis that postsynaptic voltage-gated conductances contribute to nonlinear temporal integration of synaptic input. 
EGTA, a calcium chelator, when added to the intracellular environment, will buffer intracellular calcium to a low level relative to control. Similar to experiments with QX-314, this manipulation is designed to test the hypothesis that postsynaptic processes are involved in the nonlinear temporal integration of L4 input to L2/3. EGTA, though, manipulates a set of postsynaptic conductances distinct from QX-314: those that are gated or modulated by intracellular calcium. Both of these intracellular treatments are not membrane permeable and therefore diffusion outside the targeted L2/3 cell is minimal. This property of these drugs allows us to isolate the manipulation to ion channels of the targeted postsynaptic cell as opposed to the relatively global action of bath-applied drugs.

Extracellular manipulations of AMPA and NMDA conductances with bathapplied NBQX and AP-5, respectively, allowed us to determine the dependence and modulation of this phenomenon on specific synaptic glutamate receptors. The slow component of EPSPs is attributed to the conductance of synaptic NMDA receptors. Blockade of this conductance with AP-5 should shorten the duration of EPSPs. We hypothesized that the temporal integration window of L4 input to L2/3 would be narrowed in response to this manipulation, due to the limited overlap of the faster-decaying isolated AMPA conductances.

Blockade of AMPA receptors with NBQX, on the other hand, we hypothesize will completely block or largely attenuate the synaptic input from L4 to L2/3. The magnesium-ion block of NMDA receptors requires a depolarizing stimuli to remove the magnesium block and activate the channel. In the absence of AMPA-receptor-mediated synaptic depolarization, synaptic excitation via NMDA receptors will also be attenuated or blocked. 


\section{CHAPTER 2. SIGNIFICANCE ${ }^{1,2}$}

\section{Neocortical L4 to L2/3 Temporal Integration}

The mammalian neocortex plays a critical role in the processing and perception of sensory input from the external environment. While the anatomical and somatotopic organization of the neocortex has been well described, the mechanisms of sensory information processing performed by the massively interconnected neocortical microcircuits are poorly understood. One traditional view is that information is encoded by firing rate [1-6], but there is also growing modeling and experimental data supporting the plausibility of a coding scheme based on spike-timing and population synchrony. These two coding schemes are not necessarily mutually exclusive and may operate in parallel to code for different features of the sensory input $[7,8]$.

The rodent somatosensory barrel cortex is uniquely instrumental in the study of information processing by the cortex. This region provides a vital function in rodents by processing touch information from the whiskers. Nissl stains of this region highlight histologically distinguishable columns, each of which are separated by a cell-sparse boundary in L4. Cells within L4 exhibit dendrites pruned to be contained within a column, forming dark-staining "barrels" [9]. Each L4 barrel receives principal input from a single whisker, but also responds to deflection of neighboring whiskers [9-17]. The majority of anatomical and functional descriptions of the whisker system have been gathered from experiments in rat, although hamsters and mice are also often used as animal models. For this reason, the anatomical pathway are most thoroughly described in rat, however, differences between rodents are rarely substantial.

The somatosensory information represented and processed by the barrel cortex arrives via a fairly well-described and simple pathway, with the nerve of whisker transduction separated from the cortex by only 3 synapses [18]. Each whisker is innervated by 1 to 200 afferent nerves [19,20], with cell bodies located in the trigeminal ganglion at the base of the skull. These axons merge with those of motor and other sensory nerves as part of the $5^{\text {th }}$ cranial nerve and terminate in the trigeminal nuclei of the brainstem. The transductive ends of the trigeminal nerves of the whiskers never branch and only innervate a single whisker, thus the anatomical representation of whisker input remains anatomically discrete [21]. Rudimentary coding of the sensory input is performed at the transduction step, with the deflection direction, onset, termination, and

Adapted with permission.

1 Jerome J, Foehring RC, Armstrong WE, Spain WJ, Heck DH (2011) Parallel Optical Control of Spatiotemporal Neuronal Spike Activity Using High-Speed Digital Light Processing. Front Syst Neurosci 5. doi:10.3389/fnsys.2011.00070.

2 Jerome J, Heck DH (2011) The age of enlightenment: evolving opportunities in brain research through optical manipulation of neuronal activity. Front Syst Neurosci 5: 95. doi:10.3389/fnsys.2011.00095. 
amplitude already represented by different units of this primary sensory nerve group [2124].

The primary afferent axons project to four distinct trigeminal nuclei. The majority of these axons do not bifurcate and only project to a single trigeminal nucleus, whereas the axons that do branch project to all four nuclei $[18,25,26]$. These nuclei are somatotopically organized in a similar way to L4, containing dark-staining "barreloids." Unlike the primary sensory neurons, trigeminal neurons often respond to input from more than one whisker [27-34]. Encoding and processing at this step of the input pathway is exemplified by neurons of the trigeminal nucleus principalis that are sensitive to the direction of whisker stimulation [30,31]. The encoding for input from principal and adjacent whiskers is also distinguishable, with response to stimulation of adjacent whiskers occurring with longer latencies and lower temporal precision as compared to the response to principal whisker stimulation [31].

The nucleus principalis projects to the Ventral Postereomedial nucleus (VPm) of the somatosensory thalamus from the whisker system. The leminiscal pathway of somataosenory cortical input from the whiskers is composed of synapses in the VPm and nucleus principalis of the trigeminal nuclei. Another complete whisker representation arrives in the cortex via the paraleminiscal pathway, which consists of synapses in the Posteromedial nucleus of the thalamus (PoM) receiving input from the spinal trigeminal nuclei. The lemisical and paraleminiscal pathways are believed to carry distinct features of the sensory input to the cortex. The trigeminal nuclei also project to each other and other brain regions, but these connections are beyond the scope of this work and will not be discussed further [18].

The paraleminiscal pathway projects to areas mostly oriented over boundaries of barrels, referred to in most publications as the "septal" areas. This projection does not seem to preferentially innervate a layer, with thalamocortical terminations found in all layers of the neocortex except VI. The leminiscal pathway, on the other hand, projects mainly to the barrels themselves in L4, although other layers are also sparsely innervated [35]. This differential termination pattern strongly suggests that the two inputs are differentially processed by the cortex. Synchrony in the thalamocortical inputs increases the response amplitude of L4, which in turn is believed to increase the reliability of cortical feedforward transmission [36-38], although direct experimental evidence of this notion has thus far eluded investigators.

L4, which receives the majority of thalamic sensory input, projects to $\mathrm{L} 2 / 3$, where the circuit diverges to multiple postsynaptic targets within cortex. The temporal order of barrel-cortex excitation has been elegantly investigated in vivo. In response to whisker deflection, neurons in LIV respond earliest, with L2/3 cells responding and average of 2 to $3 \mathrm{~ms}$ later. Although this pattern was repeatable and significant in response to principle-whisker stimulation, this pattern breaks-down in response to adjacent-whisker stimulation [39]. Since the L4 to L2/3 connection is the first principal intracortical connection, this step in intracortical processing undoubtedly plays a crucial role in the cortical processing of sensory input. More generalized reviews of neocortical 
connectivity and flow of excitation are numerous (i.e. [18,40-42] with the consensus being that the strongest and canonical pathway consists of the connection from Thalamus to L4, L4 to L2/3, L2/3 to layer V, and layer V to other cortical or subcortical areas. However, numerous instances of branching, lateral coupling, and alternative pathways are known. Figure 2-1 provides a visual summary of the whisker input pathway and a portion of the intracortical pathway of the barrel cortex. The presynaptic and postsynaptic components of the L4 to L2/3 connection are specifically highlighted.

Direct experimental data elucidating the coding or processing of the L4 to L2/3 connection or any other intracortical synaptic connections has thus far been indirect or stimulated, but the notion of a timing-dependent coding scheme is strongly suggested. Input to L2/3 is highly convergent, with a single L $2 / 3$ pyramidal cell receiving input from 300-400 excitatory L4 neurons [43]. In vivo recordings support the notion of cooperativity or synchrony of the convergent input, with stimulation of neighboring whiskers either facilitating or depressing the response to principle whisker stimulation in a nonlinear fashion, depending on the time interval between stimuli [44-49]. However, the physiological mechanisms and anatomical location of this implied nonlinear temporal integration are poorly understood. One study suggests that low-latency stimulation of the principal whisker and neighboring whiskers increases the probability of action potential firing in L2/3 pyramidal cells, but not cells in L4 [44], suggesting that a transformation of the sensory representation occurs at this connection. It remains to be directly investigated, however, if nonlinear temporal integration occurs here and if so, the neurophysiological mechanisms involved.

Investigation of temporal synaptic integration within the cortex has thus far been difficult due to technical limitations. A direct systematic investigation of synaptic integration across a cortical connection would require, at a minimum, quantification of both the input (presynaptic spiking) and output (postsynaptic membrane potential). For a single interlaminar neuronal connection with highly convergent input (such is the case with the L4 to L2/3 connection) this would require either:

1. The timing of action potentials in a large number of presynaptic neurons and the membrane potential of one or more postsynaptic neurons be simultaneously monitored.

2. The timing of action potentials in a large number of presynaptic neurons be controlled or manipulated with laminar specificity while measuring the membrane potential of one or more postsynaptic cells.

The recordings from option one would probably only produce meaningful results in vivo, due to the near absence of spontaneous action potentials in any part of the neocortex in the acute slice preparation. In the L4 to L2/3 connection, this relatively passive option would require a recording rig of unprecedented complexity. With hundreds of presynaptic neurons converging onto each L2/3 pyramidal cell, simultaneous monitoring of every or even a significant portion of the presynaptic population would be impossible with current technology. Optical techniques, such as voltage-sensitive dyes and calcium indicators, are quickly approaching the capability of monitoring hundreds of 


\section{Figure 2-1. Input and intracortical pathways of the somatosensory barrel cortex}

Input to the rodent somatosensory barrel cortex is separated from the sensory transduction step by three synapses. Primary whisker-input transduction neurons arising from the trigeminal ganglion project to neurons in the trigeminal nuclei, which in turn project to thalamus. The majority of thalamic input to the barrel cortex arrives in L4. The L4 (red) to L2/3 (green) connection is one of the strongest intracortical connections, but is also the first major intracortical synapse. Each L2/3 neurons receives input from 200-300 L4 spiny neurons. After the L4 to L2/3connection, the intracortical microcircuit diverges extensively. For a detailed review of intracortical connectivity beyond L $2 / 3$, see [40]. The strongest intracortical pathway beyond L2/3 is indicated with a black arrow, with some of the other known intracortical connections indicated with white arrows. 


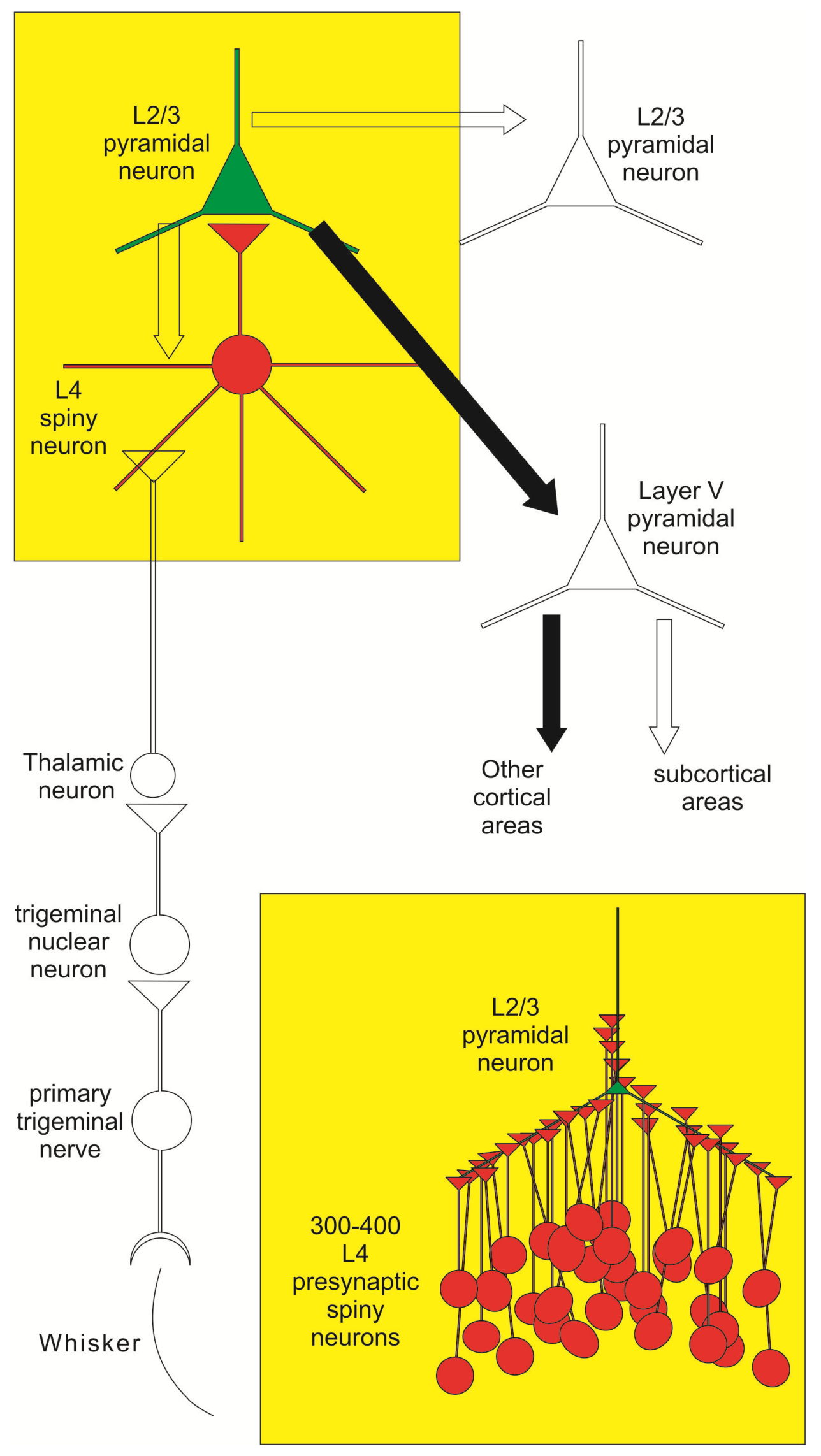


cells simultaneously, but not in any structures deeper than neocortical L2/3, with limited visual access to the majority of presynaptic neurons in deeper layers.

The acute slice preparation provides both visual and pharmacological access to both deep structures of the brain and every layer of the neocortex. The most common technique for manipulating synaptic inputs in acute slices, electrical stimulation with extracellular stimulating electrodes, has severe disadvantages. One is the number and flexibility of stimulation sites. Even with multielectrode arrays, the number of sites is typically less than 100. Furthermore, there is no flexibility in the spatial arrangement or the size of stimulation sites as electrodes are arranged on a fixed grid and the location of sites is determined by the placement and orientation of the grid. Another significant disadvantage of electrical stimulation is its poor anatomical specificity. Electrical stimulation mostly activates fibers of passage and not somata and dendrites [50] resulting in antidromic activation of neurons. This is particularly problematic in regions of the brain like the neocortex, where a high density of axons originating from multiple brain regions passes through every cortical layer. A prime example of the experimental limitations imposed by this technical weakness can be illustrated with the L4 to L2/3 neocortical connection. An investigator attempting to electrically stimulate L4 in order to investigate synaptic input to L2/3 would mostly activate axons originating from L2/3 pyramidal neurons with postsynaptic targets in layer $\mathrm{V}$, which would back-propagate to L2/3 and confound any attempts to isolate pure synaptic input from L4. Additionally, input to L2/3 not originating in L4 but with axons passing through L4 would also be strongly activated, making it impossible to determine the exact source of the input with laminar or columnar specificity.

Stimulation of presynaptic neurons with visually-targeted intracellular electrodes provides cell layer and even cell-type specificity of presynaptic action-potential induction, but is limited to no more than a few simultaneous presynaptic cells, even for the most technically-proficient electrophysiologist. However, these types of recordings have been useful in several experiments relevant to temporal synaptic integration of L4 input to L2/3. Paired L4 and L2/3 whole-cell recordings in acute slices have determined detailed parameters and statistics of the excitatory synaptic connections between L4 spiny neurons and L2/3 pyramidal cells (e.g. latency, rise time, decay time, amplitude, etc). However, this work demonstrates that even a very fast $100 \mathrm{~Hz}$ train of action potentials in a single presynaptic L4 cell is not sufficient for a synaptically-coupled L2/3 cell to reach action potential threshold [51], suggesting cooperativity, summation supralinearity, and/or additional sources of excitatory input are necessary to drive L2/3 cells to fire. Using these experimentally-determined parameters in simulations of this specific connection further support the possibility that synchrony of multiple convergent L 4 cells reliably drive L2/3 neurons [52] and more generalized simulations also support a synchrony coding scheme in large feedforward networks [53-63], although direct evidence of this hypothesis has been technically unattainable. A more detailed discussion and literature review of the L4 to L2/3 connection is provided in Chapter 5, with direct comparison to the data presented in this dissertation. 
Meaningful investigations of connections between layer-specific connections, such as the L4 to L2/3 connection, will require a spatial resolution, spatial flexibility, and anatomical specificity not possible with electrical stimuli. Following is a review of optical manipulation of neuronal activity using caged neurotransmitters, which activate neurons via glutamate receptors on somas and dendrites, avoiding the potentially confounding side-effects of axon activation seen with electrical stimulation. The success of our parallel-beam uncaging system based on Digital Light Projection technology has expanded on this capability, by allowing the precise 2-dimensional manipulation of neuronal activity, allowing us to investigate synaptic integration in a specifically-targeted interlaminar cortical circuit for the first time.

\section{Photostimulation Techniques}

Optical techniques for the bidirectional control of neuronal excitation have overcome many technical barriers, including exceptionally high spatial and temporal precision, and cell-type specificity. The following review provides a brief history of optical stimulation techniques and discusses the experimental strengths and weaknesses of a variety of different photostimulation approaches as well as the specific utility of high-frequency ultraviolet DLP photostimulation.

\section{Caged Neurotransmitters}

The development of caged compounds has had a profound effect on the biological sciences. A caged compound is formed by chemically altering a normally physiologically active molecule to include a covalently bonded functional group, often called a "protecting group" or simply a "cage". In order to qualify as a caged compound, the protecting group must have several properties. First, the cage, when chemically bonded, must render the caged molecule biologically inactive while minimizing other physiological consequences. Second, the cage must be quickly and specifically removed by photolytic cleavage. In most cases, the action spectrum of photolytic cleavage for caged compounds is in the UV spectrum. UV light, therefore, can control the active concentration of the caged molecule in a temporally (microsecond) and spatially (micrometer) precise manner. Finally, the photoreleased caged must be itself biologically inert such that photolytic activation of the caged compound results only in the action of the target molecule, and not the protecting group.

The utility of caged compounds in biology was first demonstrated with caged-ATP [64]. Neuroscience, though, has arguably gained the most from the development of caged compounds through the availability of caged neurotransmitters. In the vertebrate brain, neurotransmitters are released in complex spatial and temporal patterns at synaptic connection between neurons. With approximately $10^{11}$ neurons and $10^{14}$ synapses in the human brain, the spatiotemporal patterns of neurotransmitter release and postsynaptic activation are exceptionally complex. Photoliabile amino acid 
neurotransmitters were first synthesized in 1990 [65] and then first use in elucidating the kinetics of the nicotinic acetylcholine receptor [66].

Glutamate is the primary excitatory neurotransmitter in the brain and is consequently the most commonly used caged neurotransmitter today. Virtually every neuron type in the vertebrate and invertebrate nervous system is activated by glutamate making the use of this caged neurotransmitter universally effective. Several different caged variations are in use with each having unique properties (discussed later). The first use of caged glutamate was to study the glutamatergic activation of the giant squid synapse [67] and later for the mapping of functional circuitry in the acute brain slice preparation [68]. Before the availability of caged neurotransmitters, a light-based mapping system was proposed [69], however, this system induced action potentials by irreversibly damaging or killing the targeted cell and also stimulated axons of passage, making mapping of connections in axon-dense brain regions impossible.

Caged glutamate is currently most widely used in studies of synaptic physiology, synaptic plasticity, and functional circuitry in both normal and diseased states. The utility of any photostimulation system depends critically on the method of light modulation, which determines the spatiotemporal complexity of stimulus patterns. Caged calcium has also contributed immensely to neuroscience as reviewed by Ellis-Davies [70]. Since caged calcium is most instrumental in studies of single-cell physiology, we have omitted further discussion of its use here and have focused this introduction of uncaging techniques related to the investigation of circuits, networks, and integrative properties of neurons.

Uncaging with Ultraviolet Light. Currently, the most common scheme for photostimulation involves an objective-focused ultraviolet beam for scanning uncaging patterns. Due to a lack of commercialization of this method, multiple variations exist with no two systems being exactly identical, so this review will survey the various components of these systems in generalities.

The selection of an excitation light source is flexible and contributes greatly to the variability of system configurations. In the earliest systems and in many still used today, a xenon flash lamp directly coupled to the epifluorescence port is used to generate the photolyzing UV radiation [68]. More commonly though, lasers [71,72] are used because of their high output power, narrow emission spectrum, and other technical advantages such as fiber-optic coupling and alignment simplicity. However, even the choice of laser varies greatly, with success reported with pulsed frequency-tripled $\mathrm{Nd}: \mathrm{YVO}_{4}$, q-switched DPSS lasers [73], continuous wave argon lasers [71,72], or excimer dye lasers [74], all of which are set up to emit in the near UV (315-380nm), the wavelength band with the highest uncaging yield. Uncaging success has also been achieved with $405 \mathrm{~nm}$ visible light [75], which offers slightly decreased phototoxicity and tissue scattering at the cost of lower photolytic efficiency.

The position of the photostimulation target is modulated in a few different ways. 
With stationary scopes, a low power $(5 \mathrm{x}-10 \mathrm{x})$ objective provides a large field of view while the beam is steered into position by a pair of galvanometric mirrors and coupled to the microscope's epifluorescence port. With this method, the precise focal depth is difficult to control due to different targets in the field of view having different path lengths, so a low NA objective to generate a cylindrical beam is required.

The other common method expands the beam to fill the back aperture of a high NA, high magnification objective, generating a conical uncaging beam. The position of photorelease in the slice is modulated by a double- or triple-axis translating stage, moving the target of photostimulation relative to the fixed position of the photolysis site. Alternatively, fiber coupling of the UV source to the epifluorescence port allows the stage to be fixed while the stage-mounted microscope is moved to new targets. While this scheme is mechanically more complex and results in slower scans, systems of this type use light more efficiently, as explained in the next paragraph.

The rate at which caged compounds are released from their cage is an important parameter in photostimulation experiments as it determines the speed at which responses can be induced (i.e. the temporal precision of action potential induction). This rate is dependent on the flux density (i.e. the number of photolytic photons in a given volume) at the target stimulation site. In conical beams, the focal point of the beam has a higher flux density than any other point in the beam while in a cylindrical beam, the flux density is the same throughout the beam. Consequently, when using a cylindrical beam, greater total power is required to match the flux density at the focal point of a conical beam. With the higher total-power in cylindrical beams, the uncaging medium above the target site is subject to the same flux density and thus same high rate of caged compound release, while in conical beams, the rate is focused and optimal at the object plane exclusively. As a result, the practical lifetime of recirculated caged glutamate solution is slightly reduced in galvanometric mirror systems, which also require more-expensive higher-power light sources to achieve the same photolysis rate at the intended target.

A few unique variations of UV photostimulation systems are in current use, each with its own advantages. Introduction of galvanometric-mirror-modulated excitation light from beneath the recording chamber has eliminated the problem of excessive bath solution uncaging as the majority of the excitation light is dissipated within the slice before passing through the bath solution [76]. This system also allows very fast scans over a large field of view. Very fast scans have also been realized with an acousto-optic deflector used as a beam position modulator (as opposed to galvanometric scanners) [77]. This system also allows for the study of synaptic integration of pseudo-synchronous input, as beam position updates can occur on the scale of $50 \mu$ s per spot, although over a smaller area $(170 \mu \mathrm{m} \times 170 \mu \mathrm{m})$. While faster and technically less difficult to implement, the light throughput of acousto-optic systems is lower than galvanic mirror systems as the acousto-optic medium absorbs a significant amount of light.

The practical spatial limitation for uncaging applications is the radius of glutamate release around a focused uncaging site. Under normal circumstances, the theoretical diffraction-limited optical resolution and practical uncaging resolution (i.e. 
uncaging radius) are different by several orders of magnitude. In thick, inhomogeneous media like brain slices, the uncaging resolution is limited by light scatter and diffusion of uncaged glutamate. At these ultraviolet wavelengths, the theoretically calculated Rayleigh criterion $\left(\mathrm{r}=1.22 \lambda_{\mathrm{UV}} / 2 \mathrm{NA}_{\mathrm{obj}}\right)$ predicts an optical resolution of $<1 \mu \mathrm{m}$ while the minimum resolvable distance between uncaging spots is typically around 50-100 microns. Thus in most standard UV uncaging setups, target sites have to be spaced by 50 microns or more to activate non-overlapping populations of neurons.

While most UV photostimulation systems do not achieve single-cell resolution, they do allow investigators to resolve laminar organization of synaptic input to the postsynaptic cell. Resolution may be increased by recently described holographic uncaging systems $[78,79]$. However, this elegant but technically complex and expensive technique may have as many disadvantages as advantages. First, excitation spots cannot be chosen arbitrarily but must be generated by holographic reconstruction of fluorescent images with computationally complex algorithms, requiring genetically-tagged or dye-filled neurons to stimulate. Second, the light modulator, a liquid-crystal SLM, has a relatively slow refresh rate (approximately $60 \mathrm{~Hz}$ ), limiting its ability to present sequential spatial patterns and therefore spatiotemporally complex stimuli. Finally, while a holographic system has demonstrated the ability to induce action potentials in single cells [80], the speed and temporal precision of excitation by laser scanning systems is much greater. However, the potential for mapping synaptic connections at a single-cell spatial precision is a significant advantage and holographic photostimulation may also prove instrumental in studies of dendritic spatial integration by taking advantage of the improved resolution of direct-dendritic stimulation. Resolution has also been improved with a system utilizing the principles of total-internal-reflection (TIRF) microscopy in which an evanescent wave of UV light was used to activate caged calcium near the surface of coverslip-cultured cells [81]. However, this system probably has low utility in an acute slice preparation in which most of the viable presynaptic cells are outside the reach of an evanescent wave.

A large number of caged glutamate variants have been synthesized, including a reversibly caged glutamate [82] and a double-caged glutamate for slightly improved UV resolution at the expense of a higher power requirement [83-85]. A visible-light sensitive caged glutamate was first described in 2005 (DECM-glutamate) [86]. The decreased cost of visible-light sources is an obvious advantage and makes photostimulation systems attainable for more labs, but visible-light sensitive caged neurotransmitters are also advantageous because visible light scatters significantly less in tissue, improving the effective resolution of photostimulation. RuBi-glutamate, another visible-light sensitive caged glutamate, also has less GABA-antagonistic activity and has a greater quantum yield, allowing it to be used in lower concentrations while reducing the antagonistic effects on inhibitory networks [87]. However, the sensitivity of these caging groups to ambient room light reduces the stability in solution, requiring dark rooms and shielding of light-emitting equipment. On the other hand, the exceptional stability, the capability of being frozen, thawed and reused, the relatively low cost, and the wide availability of MNI-glutamate makes it the most commonly used caged-glutamate in both UV and 2-photon photostimulation experiments. 
Multiple uncaging systems have been described with fiber optic light delivery [88-91]. These systems have the advantage that the photolytic light does not have to be focused through a microscope objective, making them appropriate for multiple types of thicker preparations, including in vivo applications where the depth of stimulation is limited only by the length of the fiber. However, they suffer from the facts that they are highly invasive, spatially inflexible and that positioning of fibers can be very time consuming as compared to scanning systems. Additionally, the in vivo loading and replenishing of caged neurotransmitters deeper than the surface of the brain to practical concentrations is difficult if not impossible, thus fiber optic photostimulation in vivo has only be accomplished when combined with optogenetics [92,93].

Multiphoton Uncaging. Not long after Einstein elucidated the mechanism of the photoelectric effect, in which quanta of light cause electron energy-state transitions resulting in measurable electric current [94], Maria Göppert-Mayer described a theoretical excitation scheme in which simultaneous absorption of two or more low-energy photons results in electron-state transitions [95]. Proof of this theory, though, would have to wait for the advent of the laser, due to the necessary high flux density of excitatory photons. This was finally achieved in cesium vapor nearly 3 decades later [96] and applied to fluorescence excitation shortly thereafter [97].

In 1990, Denk and colleagues applied two-photon excitation to fluorescence microscopy. Several advantages exist for two-photon excitation fluorescence microscopy (2P) compared to confocal or other fluorescence microscopy techniques. In $2 \mathrm{P}$, the excitation volume is effectively confined in $3 \mathrm{D}$ at the focal point of the objective, where the flux density is at a maximum and 2-photon excitation events become several orders of magnitude more probable. In addition to matching the 3-dimensional imaging capabilities of confocal fluorescence microscopy, the infrared excitation wavelengths in $2 \mathrm{P}$ are significantly less phototoxic and damaging, allowing the observation of living tissue. The elimination of the confocal aperture combined with the relatively long $2 \mathrm{P}$ excitation wavelengths that are less susceptible to scattering, allow fluorescence $3 \mathrm{D}$ imaging in vivo at depths up to $1 \mathrm{~mm}$. Finally, $2 \mathrm{P}$ provides unmatched resolution of 3-dimensional photolysis of biologically-relevant caged molecules, first demonstrated with photolysis of DMNPE-caged ATP in a bioluminescence assay [98].

While Denk et al. speculated that improved modulation of biologically-relevant caged compounds would benefit from $2 \mathrm{P}$, many caged compounds, including most of the caged neurotransmitters, were not suitable for $2 \mathrm{P}$ photolysis due to their low propensity for $2 \mathrm{P}$ photoactivation, a measure formally called two-photon cross section with units named the Goppert-Mayer. The first such caged neurotransmitter to be efficiently released by two-photon photolysis (BHC-glutamate) was described in 1999, and was used to generate a three-dimensional, neuronal sensitivity map to glutamate [99]. Since then, multiple variations of glutamate with a two-photon-sensitive cage have emerged with each variation developed for the purpose of improving stability, improving caged inertness, increasing quantum yield, widening the action spectrum, or improving 
two-photon cross section including MNI-glutamate [100-103], MDNI-glutamate [104], and RuBi-glutamate [87]. RuBi-glutamate, mentioned earlier, in addition to being visible-light sensitive, is also two-photon sensitive at wavelengths longer than those used for MNI-glutamate, allowing for better penetration and resolution in thicker preparations. The peak output power of the commonly-used tuned Ti-Sapphire infrared lasers occurs at wavelengths closer to those used for $2 \mathrm{P} \mathrm{RuBi}$-glutamate uncaging $(800 \mathrm{~nm})$ than those used for 2P MNI-glutamate uncaging (725nm). Overall, the improved quantum yield and light-source efficiency allows RuBi-glutamate to be used at concentrations at least five times lower than MNI-glutamate. This, in combination with its lower GABA-antagonistic activity, makes it an effective tool for the study of inhibitory network activity and connectivity [87].

With 2P photostimulation, the near-infrared (NIR) photodamage threshold and limited practical concentration of bath-applied caged glutamate limits the rate of caged glutamate release at any one spot. Generally, a higher photolysis yield requires longer dwell times. While this is not a problem for the study of sub-threshold synaptic integration, action-potential initiation, and therefore presynaptic stimulation, is severely limited with two-photon systems. In one non-standard setup, a diffractive optical element was used to split the uncaging beam into several closely-spaced beamlets [105]. This system was capable of eliciting action potentials with 30 micron resolution and $5 \mathrm{~ms}$ dwell times in some cells, with 30-50 ms dwell times being typical (supplementary material for [105]). However, due to the beam multiplexing and lack of adequate controls, it is unclear whether this system is capable of single-cell specificity. Regardless, it is possible to generate neuronal connectivity maps by sequentially scanning targeted neurons with resolution and specificity better than that offered by traditional ultraviolet photostimulation systems. Similar results were found by increasing the two-photon excitation volume by reducing the effective numerical aperture of the objective [106]. In this case, it was shown that resolution, while greater than UV systems, generally stimulated multiple cells at each target.

Additional disadvantages of two-photon photostimulation are a result of the gap in current availability of near-infrared laser power and the required flux density of NIR photons for efficient photolysis of even the most easily photolyzed caged glutamate varieties. The recent description of a spatial-light-modulator (SLM) based scanless 2P microscope illustrated this particular weakness, in which increasing the number of synchronous targets decreased the response amplitude to glutamate uncaging, presumably due to a redistribution of available light power and low throughput efficiency of SLM-based systems. Even with a single cell being targeted, the initiation of action potentials required very long dwell times (100 $\mathrm{ms}$ in the author's example case) [107].

With these limitations, it is not possible to induce synchronous action potentials from two or more spatially separated presynaptic neurons. Therefore, investigating the integration of coincident input or the consequences of neuronal synchrony in large-scale circuits is beyond the capabilities of $2 \mathrm{P}$ glutamate uncaging. However, the ability to target individual spines and achieve patterned, pseudo-synchronous activation of multiple 
targeted spines in a limited field of view has been achieved with $2 \mathrm{P}$ uncaging and has been the method of choice for numerous investigations.

\section{Parallel Photostimulation Techniques}

A major drawback of most existing photostimulation techniques is that they are limited to sequential stimulation. Activity in the brain however, consists of complex spatiotemporal patterns which can only be emulated using techniques that allow massively parallel control of a large number of photostimulation sites. There is substantial experimental and theoretical evidence supporting a role of synchronous spiking activity for information processing in the brain. For example, synchronized spikes have been shown to represent essential features of visual [108,109] auditory [110,111] and gustatory [112] stimuli and to encode motor events [112,113] and also represent cognitive functions such as attention [7,114]. Currently, most of our knowledge about the generation and propagation of synchronous spike activity in the neocortex stems from theoretical studies [61,115]. Technical challenges have hindered direct and thorough experimental investigations of neuronal synchrony in biological networks. As a result, there is a large gap between theoretical predictions and our neurophysiological understanding of the mechanisms underlying neocortical synchronous spiking activity.

Photostimulation systems based on Digital Light Processing (DLP, Texas Instruments) technology allow for thousands of parallel photostimulation beams. DLP systems are built around a digital micro mirror device (DMD), an array of several hundred thousand microscopic mirrors, with each mirror corresponding to a potential photostimulation site. Each mirror can be independently positioned to reflect light in one of two directions. With the mirror in the "on" position, light is reflected through a projection lens, making the corresponding site (pixel) appear bright. Light reflected from a mirror in the "off" position is directed towards a heat sink, causing the site on the projection plane to remain dark [116]. While typically used in multimedia applications with frame rates between 5 and $240 \mathrm{~Hz}$, the array of DMD mirrors can be switched at rates of tens of kilohertz with the appropriate software and hardware controllers. DMDs thus provide high-speed parallel control of hundreds of thousands of light beams, one for each micromirror, affording the opportunity to control complex spatiotemporal activity patterns, including synchrony, over a large range of scales to emulate neuronal interactions as they occur in vivo.

The first use of DLP technology in neuroscience provided spatiotemporal control of neuronal activity in cultured rat hippocampal neurons expressing light-activated glutamate receptors at a rate of $10 \mathrm{~Hz}$ [117]. Activation of Chr2-expressing neurons with DLP photostimulation has also been used to generate glomerular input maps to mitral-tufted cells in olfactory bulb [118] and to manipulate the movement of unrestrained C. Elegans expressing Chr2[119]. A recently described Ultraviolet DLP photostimulation system has been used to investigate dendritic integration of subthreshold stimuli by uncaging glutamate at distal dendritic branch points [120]. 
However, with limited subcellular resolution, UV photostimulation mostly activates extrasynaptic receptors, which may have implications in dendritic processing.

A unique strength of DLP-modulated light is that multiple sites can be activated with perfect synchrony or arbitrarily-specified delays at a very high rate to investigate the processing of synchronous activity by individual neurons or neural networks [121]. This system, which requires the high light output of a high-power frequency-tripled ND:YAG laser, is capable of inducing action potentials with dwell times as low as $3 \mathrm{~ms}$. With the massively parallel capacity of light modulation, the synchrony of large numbers of anatomically distributed neurons can be manipulated. The control of action potential firing at high temporal resolution in multiple presynaptic neurons now allows for the investigation of important questions related to temporal synaptic integration of spatially distributed inputs and the generation and propagation of synchronous population activity in neural networks. This dissertation provides a detailed description of the design and capabilities of this system and demonstrates its utility by investigating the temporal integration of cortical L4 input to L2/3 neurons. 


\section{CHAPTER 3. MATERIALS AND METHODS ${ }^{3}$}

\section{Animal Models}

All animal experimental procedures adhered to guidelines approved by the University of Tennessee Health Science Center Animal Care and Use Committee. Principles of laboratory animal care (NIH publication No. 86-23, rev. 1996) were followed. C57BL/6 mice were housed within a breeding colony with 12-hour light/dark cycles in standard cages with ad libitum access to food and water.

\section{Acute Brain Slice Preparation}

Young (P14-P21) mice were deeply anesthetized in a 500-ml glass jar containing isoflurane-soaked gauze and decapitated. The brain was quickly removed while the head was submerged in ice-cold oxygen-bubbled dissection solution. 300- $\mu$ m coronal slices were made on a Vibratome 1500 (Vibratome, St. Louis) or a NVSL manual-advance vibroslice (World Precision Instruments, Sarasota, FL) and then transferred to warm $\left(33^{\circ} \mathrm{C}\right) 95 \% \mathrm{O}_{2}-5 \% \mathrm{CO}_{2}-$ bubbled artificial cerebrospinal fluid (aCSF). Slices were allowed to incubate for 30 minutes to 1 hour at $33^{\circ} \mathrm{C}$ before being transferred to the recording and photostimulation chamber. After the incubation period, slices were stored at room temperature in aCSF.

In early experiments, the dissection solution contained (in $\mathrm{mM}$ ): 250 sucrose, 15 HEPES, 10 glucose, $3.5 \mathrm{MgSO}_{4}, 3 \mathrm{KCl}, 1.25 \mathrm{NaH}_{2} \mathrm{PO}_{4}, 0.5 \mathrm{CaCl}_{2}$ (pH: 7.3-7.4 adjusted with $\mathrm{NaOH}, 300 \mathrm{mOsm} / \mathrm{l}$ and bubbled with $100 \% \mathrm{O}_{2}$ ). In later experiments, $250 \mathrm{mM}$ glycerol replaced sucrose. Sodium chloride, as found in normal cerebrospinal fluid, was substituted with glycerol or sucrose in order to suppress action potentials and reduce excitotoxity during the preparation procedures. In later experiments, only half of the sodium was replaced with glycerol and the $\mathrm{pH}$ was buffered with sodium bicarbonate as in normal cerebrospinal fluid (as opposed to HEPES). This and the addition of small quanities of Myo-Isonitol, Na-Pyruvate, and L-Ascorbic acid (vitamin C) resulted in a dramatic improvement in slice quality and viability and was used in the majority of experiments. This final dissection solution contained (in $\mathrm{mM}$ ): $85 \mathrm{NaCl}, 75$ Glycerol, 26 $\mathrm{NaHCO}_{3}, 10$ glucose, $3.5 \mathrm{MgSO}_{4}, 3 \mathrm{KCl}, 3$ Myo-isonitol, $2 \mathrm{Na}$-Pyruvate, $1.25 \mathrm{NaH}_{2} \mathrm{PO}_{4}$, $0.5 \mathrm{CaCl}_{2}, 0.4 \mathrm{~L}$-ascorbic acid and was bubbled with $95 \% \mathrm{O}_{2}-5 \% \mathrm{CO}_{2}$.

For the slice bathing medium, artificial cerebrospinal fluid (aCSF) was prepared to mimic the extracellular environment of neurons in the whole brain and contained (in

Adapted with permission.

3 Jerome J, Foehring RC, Armstrong WE, Spain WJ, Heck DH (2011) Parallel Optical Control of Spatiotemporal Neuronal Spike Activity Using High-Speed Digital Light Processing. Front Syst Neurosci 5. doi:10.3389/fnsys.2011.00070. 
$\mathrm{mM}): 126 \mathrm{NaCl}, 26 \mathrm{NaHCO}_{3}, 10$ glucose, $3 \mathrm{KCl}, 3$ Myo-isonitol, 2 Na-Pyruvate, 1.25 $\mathrm{NaH}_{2} \mathrm{PO}_{4}, 0.4$ L-ascorbic acid.

\section{Whole-Cell Current Clamp Recordings from Acute Brain Slices}

For all electrophysiological recordings, slices were transferred to a recording chamber and continually perfused with recirculated aCSF bubbled with $95 \% \mathrm{O}_{2}-5 \%$ $\mathrm{CO}_{2}$. Patch electrodes were fabricated from $1.50 \mathrm{~mm}$ OD $1.17 \mathrm{~mm}$ ID borosillicate glass to a tip resistance of 3-8 $\mathrm{M} \Omega$ on a Flaming/Brown micropipette puller (P-97, Sutter Instruments $\mathrm{Co}$. Novato, $\mathrm{Ca}$ ) and filled with intracellular patch-solution. L2/3 cortical pyramidal neurons in somatosensory barrel cortex were visualized and targeted for recording using 40x Hoffman-Modulation Contrast objective on an Olympus BX50WI microscope. Membrane potential signals were amplified with an AxoClamp 2B patch-clamp amplifier, digitized with a Digidata 1322a or 1440a, and recorded with pClamp 10 software (Molecular Devices, Sunnyvale, CA). The calculated junction potentials were corrected for offline.

Electrodes were mounted in a pipette holder (Warner Instruments, Hamden, CT) electrically-coupled to the AxoClamp 2B headstage and positioned with a SM-5 micromanipulator (Luigs and Neumann Ratinhen, Germany). The target neurons were approached with slight positive pressure applied to the pipette solution. Once a visible dimple formed on the cell surface, light suction was applied to form a gigaohm seal, followed by a quick burst of suction to go into whole-cell mode.

The intracellular solution contained (in $\mathrm{mM}$ ): $130 \mathrm{~K}$ - Gluconate, $7 \mathrm{KCL}, 10$ HEPES, $10 \mathrm{Na}_{2}$ P-Creatine, $4 \mathrm{Mg}$-ATP, $0.3 \mathrm{Na}$-GTP, plus, in some experiments, $10 \mathrm{mM}$ EGTA or 1mM QX-314 (Tocris).

13.5mg "caged" MNI-glutamate trifluoroacetate (Femtonics Budapest, Hungary) was added to $12.5 \mathrm{ml}$ recirculating aCSF $(2.5 \mathrm{mM}$ caged-glu in aCSF) for photostimulation experiments.

Cells under all experimental conditions were excluded from the data set if the resting membrane potential (without current injection) was more depolarized than -60 $\mathrm{mV}$ or changed more than $\pm 10 \mathrm{mV}$ during the recording. Access resistance and action potential parameters were monitored with a current-voltage response protocol $(500 \mathrm{~ms}$ current injections incremented from $-200 \mathrm{pA}$ to $+400 \mathrm{pA}$ in $50 \mathrm{pA}$ increments with 2 seconds between sweeps). With the exception of QX-314 treated cells, cells were excluded if action potentials did not overshoot $0 \mathrm{mV}$ at the end of the recording or if access resistance increased by more than $50 \%$ at any point during the recording. Since action potentials were intentionally abolished and intrinsic excitability significantly altered by QX-314, the only exclusion criteria used for these recordings was a stable, healthy resting potential. 


\section{On-Cell Recordings from Acute Brain Slices}

The procedure for on-cell recordings was similar to the procedure for whole-cell recordings except that once a visible dimple formed on the surface of a targeted L4 cell, positive pressure was released without suction. The pipette solution contained (in $\mathrm{mM}$ ): $124 \mathrm{NaCl}, 2.5 \mathrm{KCl}, 2 \mathrm{CaCl}_{2}, 1 \mathrm{MgCl}_{2}$, and $10 \mathrm{HEPES}$. An Axopatch-1D in passive current-clamp mode was used to amplify signals from the second electrode in paired recordings.

\section{Digital Light Projection Electronics and Software}

Single-photon uncaging of most photolabile compounds requires the use of ultraviolet light. We thus employed an ultraviolet-compatible version of the DLP Discovery 3000 kit (Digital Light Innovations, Austin, TX, USA). The digital micromirror device (DMD) communicates with a computer (Windows PC) via a USB interface board (ALP-3 High-Speed, Vialux, Germany). The USB kit includes a CD with a dynamic link library (DLL) containing $\mathrm{C}++$ functions for controlling the mirrors. The supporting documentation of this DLL describes the functions for loading sequences, starting and stopping sequences, and setting the timing of frame changes. A graphical-user-interface (GUI) for the generation of 2-dimensional stimulus patterns was programmed in C\# using Visual Studio 2008 Professional (Microsoft). USB control of the DLP system uses commands embedded in the ALP-3 DLL. Since these functions were written in $\mathrm{C}++$, the functions were imported and marshaled into their own wrapper class. Importing and marshalling of ALP functions requires correct C\# equivalents of $\mathrm{C}++$ data types.

The $\mathrm{C}++$ sample code provided with the ALP-3 documentation has many $\mathrm{C}++$ precompiler directives (\#define) that give meaningful names to frequently used constants, such as return codes and parameters for functions. Visual Studio's C\# compiler does not make use of precompiler directives; these constants must be declared as const ints (a c\# data type) to work. The ALP-3 high-speed documentation provides a complete list of constants formatted as precompiler directives.

Several built-in functions of the ALP-3 DLL require a specific procedural implementation and in many cases require that the calling code includes intentional delays, using timers or processer pauses between function calls in order to allow for USB communication delays. For example, the loading of projection sequences requires that the ALP-3 device is not actively loading or projecting other sequences, so all such operations must be programmatically stopped. The device must also be freed from previous initializations and reinitialized in order to obtain a valid programmatic handle to the GUI.

The ALP-3 kit also includes enough RAM for buffering up to 1365 XGA (1024 X 768) binary (black/white) frames. Loading sequences to the RAM of the ALP-3 board required that sequences be represented as 3-dimensional byte arrays. To take advantage of the graphical capabilities of the Microsoft .NET framework and C\#, photostimulation 
sequences are generated and graphical manipulations are performed on bitmap objects with .NET functions. The bitmaps are then converted, one at a time, into 3-dimensional byte arrays and loaded into the RAM of the ALP-3 board. Each sequence requires that RAM on the ALP-3 board be explicitly allocated. Examples of the correct syntax for importing DLL-embedded functions, declaring constants, ALP-3 initialization, allocating ALP-3 RAM, performing graphics manipulations, loading sequences, and starting and stopping projection sequences can be found in the Appendix.

The planning, execution, and on-the-fly modification of stimulus patterns necessitates the need of an intuitive and flexible graphical user interface for quickly and efficiently designing spatiotemporal patterns. Generally, investigations utilizing the DLP photostimulation system have unique experimental needs and several different forms within the multiple-document interface provide this flexibility. For the majority of experiments performed for this dissertation research, a form that utilizes size-adjustable photostimulation targets on a grid with 32-pixel spacing $(86 \mu \mathrm{m}$ with a $5 \mathrm{X}$ projection objective) were activated sequentially or synchronously. This pattern type is useful for presynaptic stimulation of a whole-cell recording. However, significant effort went into designing GUI forms for spatiotemporally complex direct-dendritic stimulus patterns. These patterns included random activation of sites (to simulate background noise) and different degrees of population synchrony. These patterns included hundreds of sites, cryptographically-strong random activity, and were computationally intensive, generally requiring about 20 minutes of processing time to generate on a late-generation PC. This form is still functional and may be useful for future experiments.

The USB accessory board outputs a TTL signal with the "high" (5-volt) state indicating that a frame is being actively projected while the "down" ( 0 -volt $)$ state indicates either an inter-frame period or an idle DMD. The first high state pulse, i.e., the onset of the first frame of a stimulus sequence, was used to temporally align neuronal membrane potential recordings to the photostimulation sequences.

\section{Digital Light Projection Optical and Mechanical Design}

The projection and illumination optics were assembled and aligned within a custom optical cage (60 and $30 \mathrm{~mm}$ cage systems, Thorlabs; additional parts machined by UTHSC biomedical instrumentation machine shop). In order for the DLP photostimulation optics to be incorporated into the electrophysiology rig for projection through the bottom of the perfusion chamber, the microscope's condenser, and the photostimulation optics had to be mounted onto precision sliding rails to position either the condenser or the DMD projection optics underneath the chamber. The condenser was positioned under the perfusion chamber while patch-clamp recordings from neurons were established under microscopic guidance. After a stable recording was established, the condenser was replaced by the projection optics. Start and end points of rail movements were precisely determined by adjustable mechanical stops. 
We illuminated the full area of the DMD with UV light using a pulsed 3.5-watt UV laser (frequency-tripled, q-switched, ND:YVO4, $355 \mathrm{~nm}$ laser, 3530-30, DPSS laser, Santa Clara, CA, USA). The raw beam was expanded and homogenized with a custom refractive beam shaper (StockerYale, Salem, NH, USA). The expanded beam was folded to the appropriate illumination angle with a high-power UV mirror (15087, Lambda Research Optics, Figure 3-1), at which point the light homogeneously illuminated the surface of the DMD. DMD micromirrors in the "off" position reflected light to a light trap consisting of a piece of aluminum painted with black permanent marker. Micromirrors in the "on" position reflected light orthogonal to the surface of the DMD through the projection optics to the perfusion chamber. The projection optics consisted of a tube lens (P4033741038, Zeiss), a 90 cube-mounted turning mirror (CM1-F01, Thorlabs) and a high NA, low magnification microscope objective (Fluar 5X, NA 0.25, Carl Zeiss, Germany), which focuses the image of the UV-illuminated on-positioned mirrors onto the neuronal tissue through a UV-permissive quartz-glass coverslip (SPI supplies, 01015-AB, West Chester, PA, USA). The coverslip forms the bottom of the recording chamber on which the slice rests.

\section{Data Analysis}

The DLP photostimulation control program automatically generated a log (text file) that allowed the automatic renaming and organization of data files with a second custom Visual Studio C\# program. The intracellular electrophysiology data was visualized, filtered, and analyzed in Clampfit 10, part of the software package that comes with the pClamp data acquisition program (Molecular Devices, Sunnyvale, CA). On-cell extracellular spike trains were visualized and analyzed using Spike 2 (Cambridge Electronic Design, Cambridge, England). Noise and electrical interference were filtered out with band-pass and notch filters as necessary.

\section{Calculation of Summation Ratio and Supralinearity}

Summation ratio (SR), a computed measurement based on the area under the curve of membrane potential traces, was used to compare excitatory intracellular membrane potential responses to photostimuli. The traces measuring the response to photostimuli were 2-seconds long, with $200 \mathrm{~ms}$ before the photostimlus used as a baseline. For measuring raw area under the curve, the signal was baseline adjusted by subtracting the average membrane potential during the stimulus-preceding $200 \mathrm{~ms}$. The signal was then rectified so that negative membrane potentials were forced to a value of 0 . Taking the integral of this rectified, baseline-adjusted signal allowed us to quantify the positive-going, excitatory response. Without this adjustment, measuring the area under the curve of responses with long-lasting after-hyperpolarizations or responses with negative baseline drifts would result in misleadingly small positive values or artifactually negative values. 


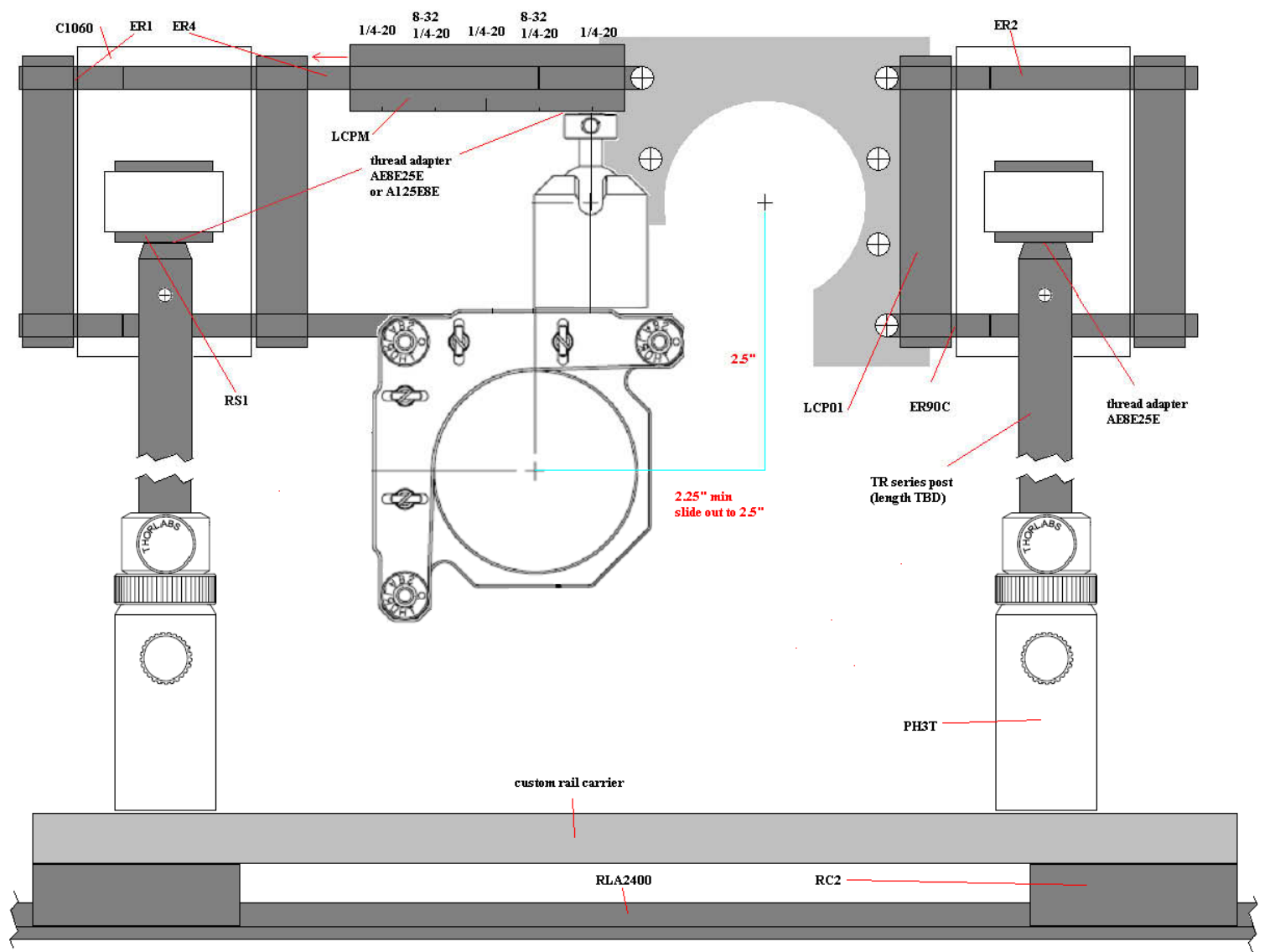

Figure 3-1. DLP illumination mirror and mechanical design

Schematic diagram of the illumination mirror position and mechanical cage that supports the DLP photostimulation system. The center of the DMD is indicated with a plus sign $(+)$. The mirror is positioned 45-degrees off center and reflects light at an angle out of the page and towards center of the DMD. The primary axis of the light reflected off the DMD is directly into the page, perpendicular to the surface of the DMD. The projection optics (consisting of the tube lens and objective) are not shown. 
For the ten sequential traces, the integral was computed from $200 \mathrm{~ms}$ (onset of photostimulation) to $350 \mathrm{~ms}$ with the built-in pClamp measurement routines, resulting in an area value in units of millivolt*milliseconds. Because the EPSPs were generally isolated and rare after this integration window, longer integration windows were unnecessary. The area values from each of the ten sequential sites were added to produce the divisor of the computed SR. The addition of area under the curve from ten traces in this way results in an integral with a time-base that is $1500 \mathrm{~ms}$ long (i.e [ten sites] * [150 ms each]).

The dividend was the similarly obtained area under the curve of the membrane potential response to patterned stimuli. This integral, however, was computed from 200 ms (onset of photostimulation) to $1700 \mathrm{~ms}$ in order to keep the time base the same as the divisor. Additionally, patterned stimuli had input arriving over a much larger time window and the integration window had to be appropriately adjusted in order to account for this. A figure illustrating the process for computing the SR can be found in Chapter 4. Traces representing the arithmetic sum of sequential stimuli were generated with arithmetic routines built in to Clampfit. The integral of these arithmetically-generated traces is both analytically and empirically identical to the summed integrals of the individual sequential traces. These summed traces, however, are more useful for visualizing SR and synaptic integration linearity.

\section{On-Cell Action-Potential Analysis}

Action potentials from intracellular data were unambiguously visually identifiable and were counted manually. On-cell spikes were easily distinguishable as $1 \mathrm{~ms}-3 \mathrm{~ms}$ long positive-going deflections in the voltage signal, often followed by a slightly longer negative component, representative of the after hyperpolarization. The peak of these spikes was marked with a manually-adjusted threshold in Spike2.

\section{Statistics}

Measurements were tabulated in Microsoft Excel, with calculated measurements, means, and standard errors calculated using common Excel formulas. Statistical tests for significance (ANOVA) were performed using SAS 9.2 (SAS Institute Inc., Cary, NC).

Each cell was presynaptically simulated with sequential and spatiotemporal presynaptic stimulation for two (control) or four (before and after pharmacological manipulation) identical trials. To minimize glutamate desensitization and/or plasticity, there was 30 seconds recovery time between each photostimulus. In all cells, the interstimulus intervals tested were (in ms): 0, 1, 2, 5, 10, 15, 20, 30, 40, 50, 100 and 30 -second sequential scans. The timing and order of these stimuli was constant and controlled programmatically, resulting in recordings that were about 1-hour long. 
The independent variables in a multiple-factor ANOVA were cell, interstimulus interval (ISI), and treatment. Comparisons of SR between intracellular treatment cells (EGTA, QX-314, $\mathrm{n}=4$ each) and control cells $(\mathrm{n}=20)$ were performed using an unbalanced repeated measures Dunn's multiple comparison test with treatment nested within cell, comparing the treatment groups against the control group. Comparisons of SR between extracellular treatment groups (AMPA, NMDA, $n=4$ each) were performed with a paired repeated measures ANOVA with the first two trials acting as an in-cell control compared to the second two treated trials. P-values of 0.05 or less were considered significant and marked with an orange asterisk in figures plotting ISI vs. SR. 


\section{CHAPTER 4. RESULTS ${ }^{4}$}

\section{System Design and Optical Performance}

The key component of the DLP-based photostimulation system is the DMD (Texas Instruments). A DMD is a matrix of thousands of microscopic mirrors with each mirror serving as a single pixel in multimedia projectors and lithography systems. The mirrors have two tilt angles (Figure 4-1A). These "on" and "off" positions reflect light toward the projection target or toward a light trap, respectively (Figure 4-1B). Each mirror's tilt angle is independently controlled. Here we use the DMD to project 2-dimensional photostimulation patterns onto neuronal tissue. Multiple 2-dimensional frames are presented in sequence to generate spatiotemporal photostimulation patterns. The system allows a maximum frame rate of 13,333 frames per second.

Our design introduces photostimulation light via a separate optical path from beneath the perfusion chamber. This generates two major advantages over stimulation through the microscope objective: (1) It provides optical access to a much larger area of the slice and (2) it minimizes the uncaging of glutamate in the bath solution above the slice because photons are first absorbed within the slice. The projection objective demagnifies the image of the mirrors by a factor of 5, resulting in a projected pixel size of $2.7 \mu \mathrm{m} \times 2.7 \mu \mathrm{m}$. The objective generated a conical excitation profile with a high light-flux density at the focal plane within the brain slice and diffuse light above the slice. As configured, this system allows optical control of neuronal spike activity within a 2.76 $\mathrm{mm} \times 2.07 \mathrm{~mm}$ area of the slice. Figure 4-2A shows a typical neocortical coronal slice overlaid with the projection of a grid. The grid pattern uses about $25 \%$ of the DMD surface and is large enough to cover many neighboring barrels and all six layers of the barrel cortex of a mouse.

A relatively high light intensity is required to photolytically release glutamate at concentrations sufficient to excite neurons. UV light power density, measured with an optical power meter (Thorlabs, Newton, NJ, USA) at the focal plane of the projection objective, was on average $250 \mathrm{~mW} / \mathrm{mm}^{2}$. This corresponds to a total absorption of $40 \%$ of the optical power in the projection light path, the majority of which is absorbed by the objective. Power was measured after $1-\mathrm{h}$ of laser warm up before an experiment and immediately following an experiment and remained stable ( $<2 \%$ power change). The $x-y$ optical resolution of this system was demonstrated by projecting patterns onto a quartz coverslip painted with blue permanent marker placed painted side down in the perfusion chamber. The UV light bleached permanent imprints of the projected DMD image. Single-pixel-wide bars were distinguishable when separated by 3pixels, suggesting a maximum optical resolution of $8.1 \mu \mathrm{m}$ (Figure 4-2B). Using the peak wavelength of the

Adapted with permission.

4 Jerome J, Foehring RC, Armstrong WE, Spain WJ, Heck DH (2011) Parallel Optical Control of Spatiotemporal Neuronal Spike Activity Using High-Speed Digital Light Processing. Front Syst Neurosci 5. doi:10.3389/fnsys.2011.00070. 

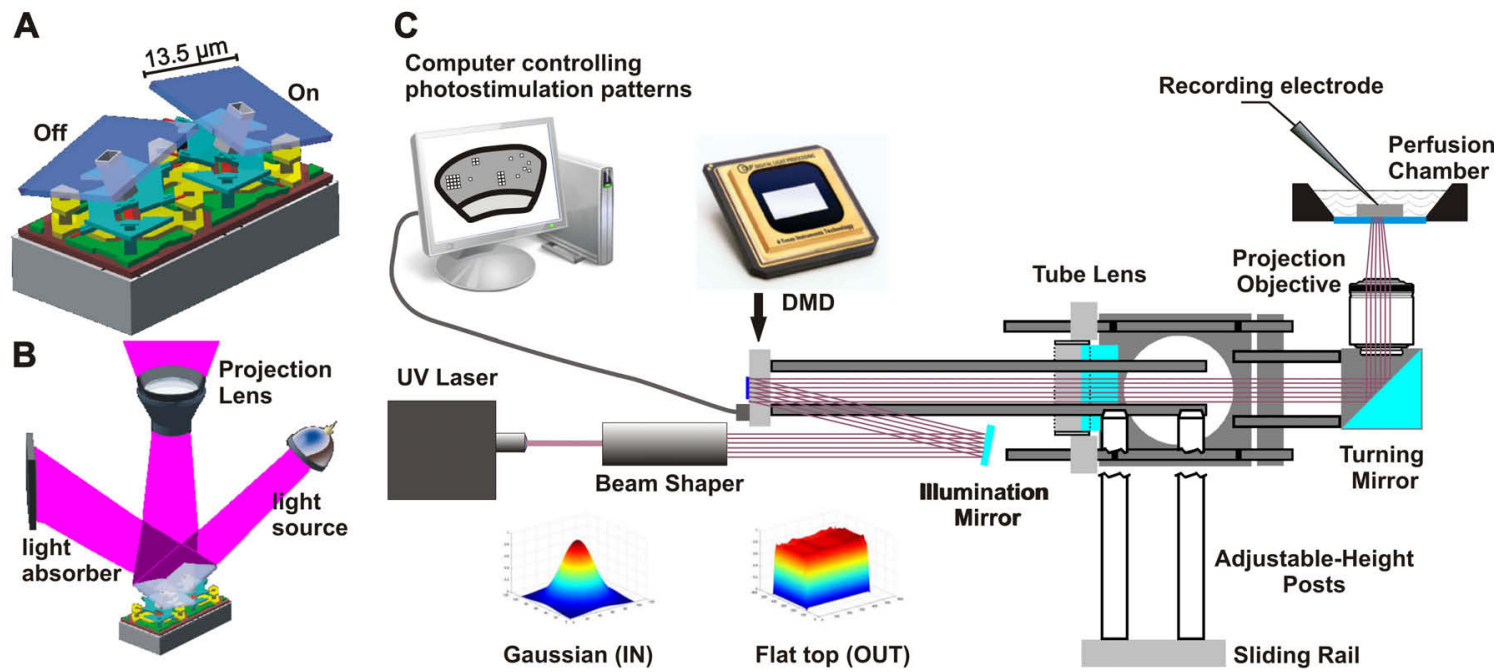

Figure 4-1. Principal of DLP neuronal photostimulation

(A) Two DMD mirrors with one mirror (left) in the "off" and the other (right) in the "on" position (illustration adapted with permission from Texas Instruments). (B) Illustration of a two-mirror DLP system. Each micromirror reflects the light either towards the projection optics (left mirror in the bottom illustration) or towards a light absorber (right mirror) (illustration adapted with permission from Texas Instruments). (C) Schematic drawing of the light path for the DLP photostimulation system using a UV laser, a "beam shaper" (Flat-Top Generator, Stocker Yale, NH) and the DMD to reflect UV light onto stimulation sites on the slice. The "beam shaper" alters the spatial intensity profile of the laser beam from Gaussian to a rectangular "flat top" (color illustrations below beam shaper) to ensure homogeneous illumination of the DMD. 


\section{Figure 4-2. Optical performance of the DLP photostimulation system and resolution of glutamate uncaging}

(A) Overlay of a grid projection using approximately $25 \%$ of the DMD surface and a photomicrograph of a neocortical slice demonstrating the area of slice tissue that can be covered by DMD photostimulation. The inset shows the center of the microscope's field of view at high magnification with a patched neuron. The position of the projected DMD image and the center of the field of view were fixed, allowing alignment of photostimulation with the patched cell. (B) Demonstration of 3-pixel $(8.1 \mu \mathrm{m})$ optical resolution of the DLP photostimulation system. The DMD-reflected UV light is projected onto a blue-painted coverslip bleaching the paint. The top panel shows 1-pixel wide bars, separated by gaps of the indicated width. The trace at the bottom plots the average numerical gray levels along the horizontal axis. (C) With $2.5 \mathrm{mM}$ MNI-glu in the bath solution, photostimuli of $8 \times 8$ pixels (micromirrors) induce action potentials (red traces) in a $\mathrm{L} 2 / 3$ pyramidal cell at sites aligned anatomically to the cell body or dendrites. The traces represent the membrane potential response elicited by photostimulation at each site. (D) Expanded views of the traces marked with numbers in $\mathbf{C}$, showing action potentials elicited by photostimulation of the distal dendrite (2) and soma (1) of a L2/3 pyramidal cell as well as the absence of both subthreshold and suprathreshold responses to photostimulation of adjacent sites $(3,4)$ on either side of the apical dendrite. The timing and duration of photostimulation $(10 \mathrm{~ms})$ is indicated with a black bar beneath the traces. (E) Synaptically induced responses (5) occur several milliseconds later than responses to direct stimulation (1). 

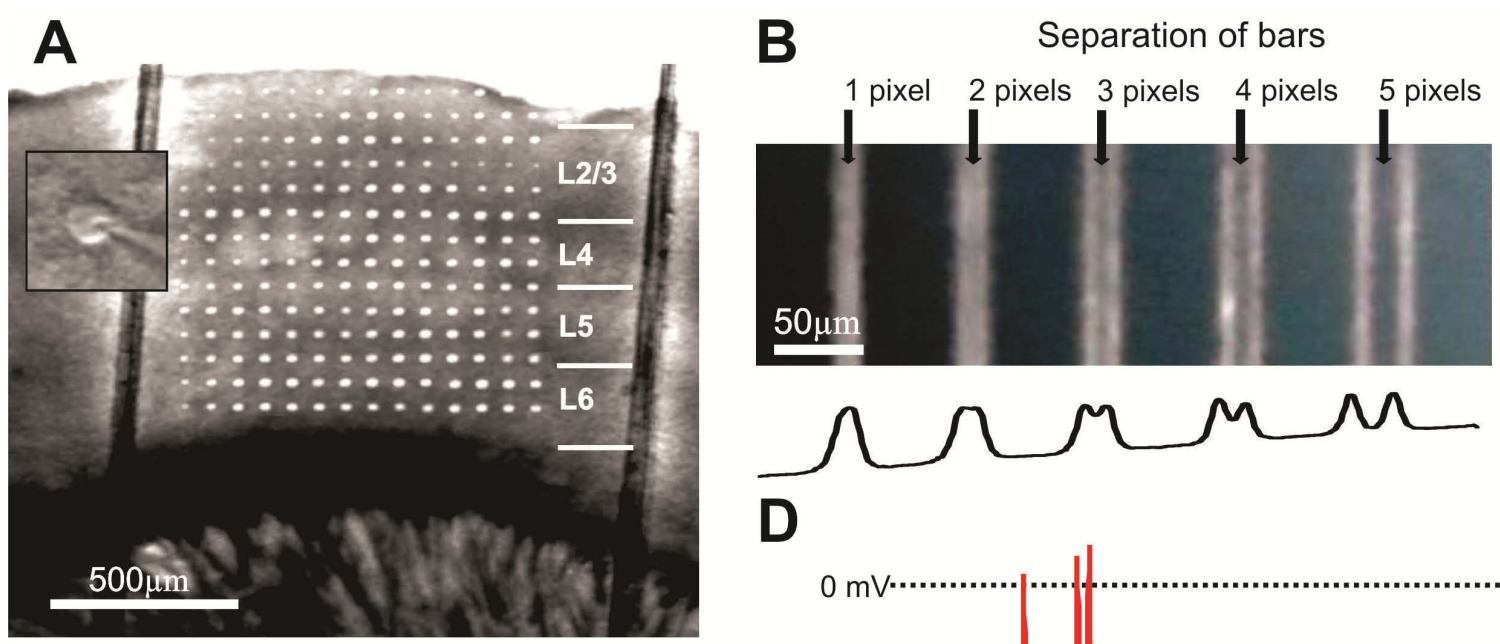

C

2.5mM MNI-glutamate
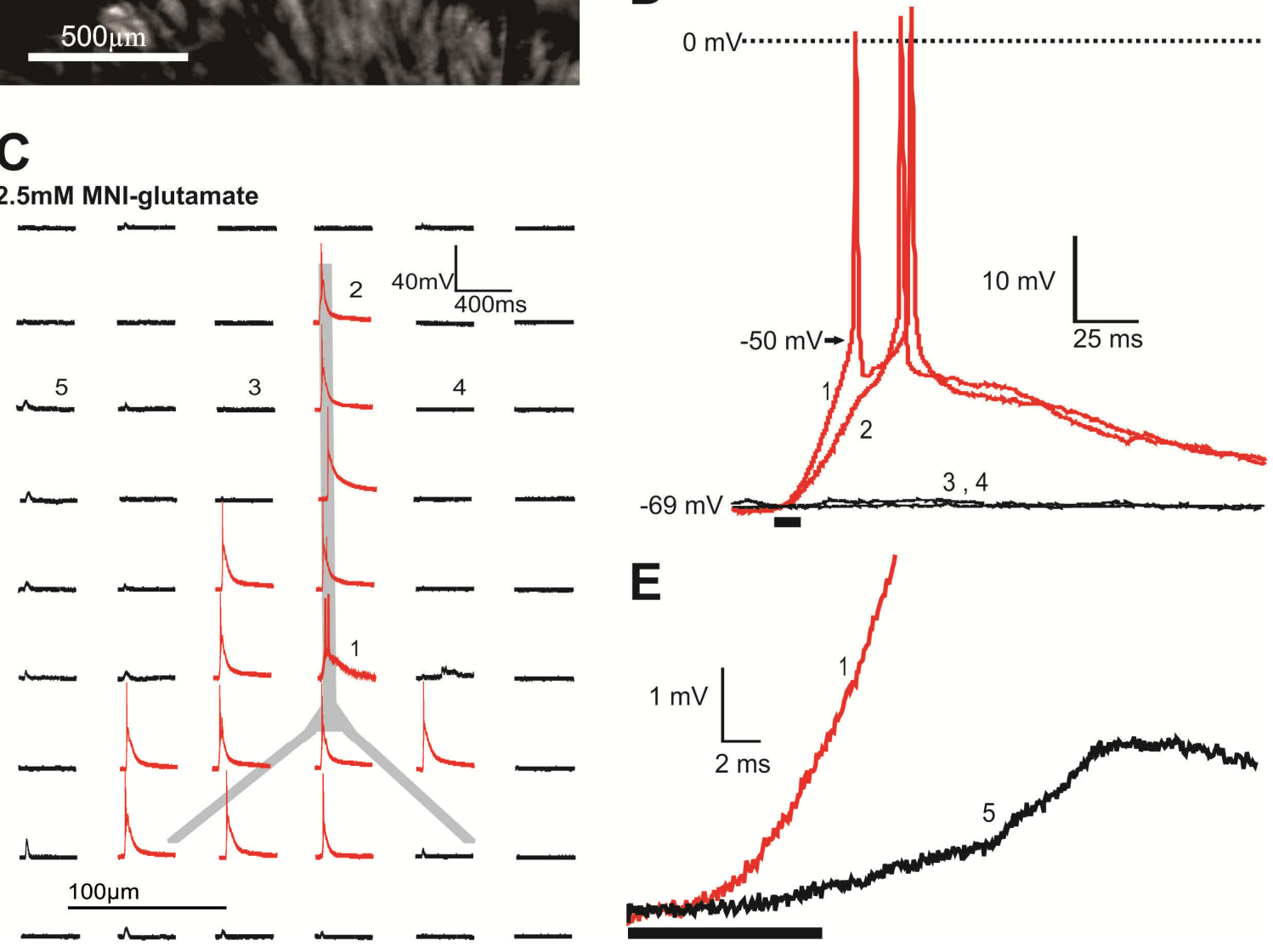
laser $(355 \mathrm{~nm})$ and the numerical aperture of the projection objective $(0.25)$, The Rayleigh criterion calculates the theoretical resolution:

$$
\mathrm{R}=1 /(2 * \mathrm{NA})=335 \mathrm{~nm} /(2 * 0.25)=710 \mathrm{~nm} .
$$

When the bars are separated by 3 pixels, the minimum of the first diffraction order of one bar corresponds to the maximum of the first diffraction order of the neighboring bar. Thus, the empirically determined Rayleigh criterion is 3 pixels $(8.1 \mu \mathrm{m})$, which is larger than the calculated optical resolution.

The technical limits of photostimulation patterns in this system are set by the maximum number of frames in a sequence (1365), the number of pixels or mirrors on the $\operatorname{DMD}(1024 \times 768)$, pixel size $\left(2.7 \mu \mathrm{m}^{2}\right)$, and the maximum frame rate $(>13 \mathrm{KHz})$. Within these constraints, any sequence of 2-dimensional frames can be projected as UV photostimulation patterns. For any one sequence, the frames can be repeated in any order determined by the experimenter. This includes repetitions of the same frame as often as desired. The frame sequence can be played back with any frame rate and any interval between frames down to the maximum temporal resolution of the system (limited by the USB controller's speed).

\section{Determining Physiological Resolution of DLP Photostimulation}

Ultraviolet light scatters significantly in living brain tissue and the bathing solution. Since scattered light also uncages glutamate, the actual physiological resolution of the photostimulation system is considerably lower than the maximal optical resolution measured with homogenous, thin projection media. The spatial resolution for the optical control of spiking activity was determined with $2.5 \mathrm{mM}$ caged 4-methoxy-7-nitroindolinyl-caged-1-glutamate (MNI-glu) in the bath solution. In order to maximize the probability of eliciting spike responses we used the highest concentration of caged glutamate reported in the literature so far. A concentration of $2.5 \mathrm{mM}$ is widely reported to be stable in solution with no detrimental effects to cells (i.e., [122]) and is the only concentration we used in experiments. No noticeable changes in photostimulation efficacy or strength was apparent for the length of the experiment, given that each site was allowed to recover by not being stimulated more than once every $30 \mathrm{~s}$. This suggests a relatively stable concentration of caged glutamate in the bath for the length of the experiment.

The cell body and direction of the apical dendrite is easily seen with IR Hoffman-modulation contrast enhancement (Figure 4-2A inset). The position of the projected pixels relative to the center of the field of view of the microscope is fixed. The position of the soma relative to the DLP sites can thus be determined visually by centering the cell on a cross hair in the microscope's visual field. A grid of $21.6 \mu \mathrm{m} \mathrm{x}$ $21.6 \mu \mathrm{m}$ ( 8 pixels $\mathrm{x} 8$ pixels) photostimulation sites with $86 \mu \mathrm{m}$ (32-pixel) spacing anatomically oriented over the soma or dendrites of L $2 / 3$ cells reliably induced action potentials, while sites not aimed at the soma or dendrites did not (Figure 4-2C, 
Figure 4-2D, Figure 4-2E). With this spacing, one site could induce a suprathreshold membrane potential response and an undetectable response in the neighboring site $(\mathrm{n}=3$ cells). This suggests that the spacing used in these experiments, $86 \mu \mathrm{m}$, is sufficient to separate the radii of glutamate release due to activation of a single site, creating clearly distinct physiological responses. This further suggests an upper limit of the physiological resolution of $86 \mu \mathrm{m}$, which is comparable to most other UV uncaging systems designed to induce action potentials in presynaptic cells for circuit mapping purposes $[68,123]$.

In order to determine whether action potentials were elicited by direct stimulation of the recorded cell or via synaptic input from activated presynaptic cells we compared the time courses of postsynaptic potentials. Analysis of excitatory postsynaptic responses elicited by action potentials in presynaptic neurons (Figure 4-2D, Figure 4-2E) shows that synaptically elicited excitatory postsynaptic potentials (EPSPs) have post-stimulus onset times several milliseconds later than responses elicited by stimuli directly targeting the cell. The two response types were thus clearly distinct and action potentials were the result of direct photostimulation of the recorded cell. If subthreshold responses are of interest, as in many other uncaging applications, the physiological resolution will likely be higher due to the potential for using smaller groups of mirrors and shorter dwell times. However, the particular strength of the described method is that the system can be used to control spatiotemporal spiking activity. We thus focus on the parameters relevant for eliciting spike firing in the targeted neurons.

\section{Spatiotemporally Complex Direct-Dendritic Photostimulation}

The initial proposed use of the DLP photostimulation system were experiments in which the dendritic tree of cortical pyramidal neurons was directly stimulated with spatiotemporal patterns that had random background noise and populations of synchronous inputs. These experiments were designed to test the hypothesis that pyramidal neurons would be more sensitive to higher degrees of input synchrony.

Significant effort went into programming the control software for performing these experiments. These patterns consisted of random activation of photostimulation sites (to simulate background noise) and a set of sites used to simulate different degrees of input synchrony. The random background activity used a cryptographic random-number generator to specify the activation timing of hundreds of sites oriented over the dendritic tree. Another cryptographic random number generator specified when synchronous events occurred and a third cryptographic random generator determined the spatial arrangement of synchronous sites. Cryptographically-strong random number generators were necessary to avoid unintentional patterning or oscillation of the stimuli. Generating these patterns was very computationally intensive, generally requiring about 20 minutes of processing time to generate on a late-generation PC. For this reason, patterns had to be designed in advance of the experiments and were difficult to adjust after an experiment was started. While this program is still functional, it likely needs simplification in order to be practical for future experiments, which most likely would require fast on-the-fly modification of stimulus patterns. 
The cells did not tolerate the stimulus well. The majority of cells (about 80\%) stopped spiking or died within a second of starting the stimulus patterns. The cells' intolerance of repeated long-exposures to damaging ultraviolet radiation is one possible explanation. However, it is the author's belief that excessive calcium influx through mostly extrasynaptic NMDA receptors was the primary reason for the limited longevity of photostimulated neurons. Membrane potential responses were unusually long depolarizations (on the order of 10 seconds), supporting the calcium influx hypothesis. The addition of EGTA to the intracellular electrode solution slightly alleviated the problem, but not enough to obtain repeatable responses and also directly altered the integrative properties of the neurons from which recordings were obtained. If overactivation of NMDA receptors was indeed the cause, pharmacological blockade of NMDA receptors may allow future investigators to directly-stimulate the cell with spatiotemporally complex patterns. These types of experiments may directly compete with whole-cell recording protocols using noisy or complex somatic current injections, which are limited to single-site input, and not input that is dendritically distributed as it occurs in vivo.

Responses from the remaining cells were entirely unrepeatable; membrane potential responses to identical stimulation patterns varied very greatly in strength between cells. Several potential factors affecting the response strength are technically impossible or difficult to control, including: dendritic morphology, cellular electrotonic compactness, cell depth within the slice, and local concentration of caged neurotransmitter. Since adjustment and tuning of the intensity and frequency of the stimulus patterns to account for and normalize this discrepancy could not be performed on the fly, simplification of the stimulus patterns and control interface may be necessary to make these experiments fruitful. Figure 4-3 provides an example response of layer $\mathrm{V}$ pyramidal neuron to direct dendritic stimulation. This cell fired sparsely in response to 20 $\mathrm{Hz}$ patterns. Other layer $\mathrm{V}$ neurons, however, would respond to the same pattern by tonically depolarizing $-30 \mathrm{mV}$ to $-10 \mathrm{mV}$ and taking several minutes to recover, illustrating the extreme variability in the response to these patterns.

Another weakness of these experiments was the limited subcellular resolution and targetability of UV uncaging. UV light scatters significantly in an acute brain slice, imposing a technical limit on the useful spatial resolution of neurotransmitter uncaging. Many argued that the majority of the sites that were stimulated contained mostly extrasynaptic receptors, were missing the cell entirely, or were in fact inducing action potentials in neurons presynaptic to the targeted cell. Almost every existing UV uncaging system is used to induce action potentials in presynaptic cells for circuit-mapping purposes and not for direct-dendritic stimulation. Knowing that we would never be able to compete with the subcellular spatial resolution of 2-photon systems, we dramatically altered our scientific goals, which necessitated a new experimental plan and an overhaul of the control software. 

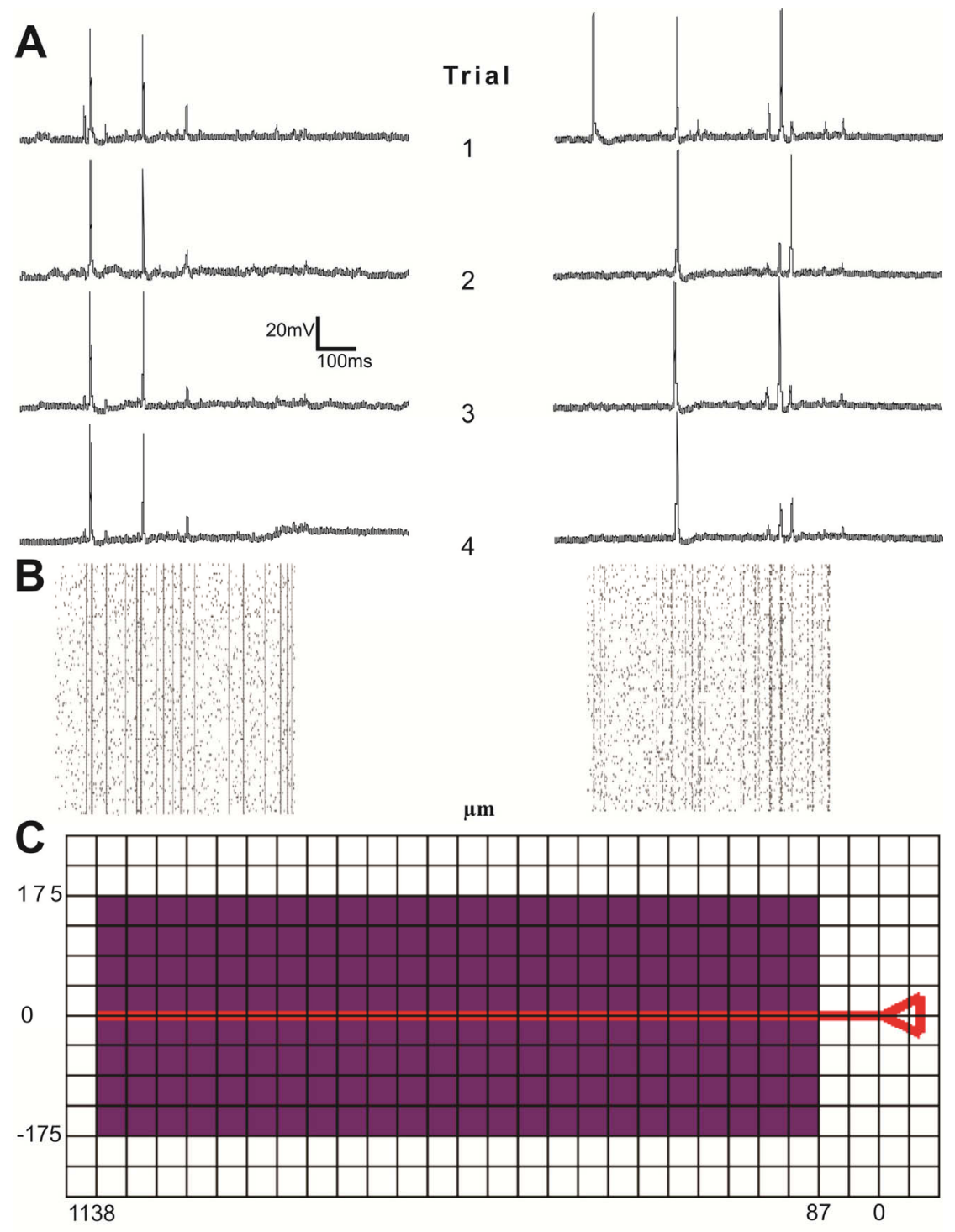

Figure 4-3. Direct dendritic photostimulation of a neocortical layer $\mathrm{V}$ pyramidal neuron

(A) Four trials of layer $\mathrm{V}$ pyramidal cell membrane potential responses to photostimulation patterns with 192 sites independently controlled sites. (B) Raster representation of the spatiotemporal stimulus pattern with each horizontal line in the raster representing the activation time of the corresponding site. The spatiotemporal patterns consist of random background activity and a population of inputs that are synchronized. Each site is activated (turned on) for $10 \mathrm{~ms}$ at an average frequency of 20 $\mathrm{Hz}$. On the left, $100 \%$ of the synchronous population turns on in a synchronous frame, which is indicated by a vertical bar in the raster plot. On the right, only $50 \%$ of the synchronous population is on during a synchronous frame. (C) Part of the graphical user interface for generating the patterns, showing the targeted stimulation area over a layer $\mathrm{V}$ cortical pyramidal cell. 


\section{Photostimulation-Induced L4 Excitatory Synaptic Input to L2/3}

The following set of experiments was designed to characterize the photostimulation-induced synaptic activity of the L4 to L2/3 connection within barrel cortex. Whole-cell patch-clamp recordings were obtained from L2/3 pyramidal cells with normal intracellular pipette solution (see Materials and Methods). Cells were subject to strict exclusion criteria (see Materials and Methods) to obtain a data set from cells that remained healthy for the full length of a recording. This data set only included cells with healthy resting potentials and overshooting action potentials. For all of the included cells, the whole-cell recordings remained passive (no current injection) during all periods of photostimulation. Periodically, cells were subjected to current-protocols to obtain IV curves for the purposes of monitoring access resistance and firing properties. Recordings from cells that did not meet these exclusion criteria were purposefully stopped to save time during an experiment and excluded from the analysis, leaving a healthy representative group of 20 cells included in the analysis.

Using the graphical user interface, ten sites within L4 were selected for photostimulation (Figure 4-4A). The sites consisted of binned groups of 8x8 DMD pixels focused on the bottom of the slice chamber. The sites were separated by $86 \mu \mathrm{m}$ in order to maintain clear separation of the glutamate-uncaging radii. In this way, activation of each site uncaged glutamate over non-overlapping populations of L4 cells and each site could be considered independent. The position of photostimulation sites within L4 was confirmed in video recordings filmed with a CCD camera (Hamamatsu C2400) through the microscope's 5X objective. Light flashes for each UV stimulus were clearly visible on a video monitor connected to the CCD camera. The UV laser emits light in the visible spectrum, albeit at low intensities, and the slices auto-fluoresce under UV illumination. Both components are likely to contribute to the visibility of the light stimuli in the CCD video system. Photostimulus onset times (signaled by a TTL-format DLP output) were digitized together with the membrane potential data. Patch-clamp recordings were obtained from L2/3 cells between $100 \mu \mathrm{m}$ and $200 \mu \mathrm{m}$ away from the edge of L4 in order to avoid direct stimulation of $\mathrm{L} 2 / 3$ cells by photorelease of caged glutamate anywhere near L2/3 and also minimize differences in L4 to L2/3 connection probabilities between cells.

Activation of each individual L4 photostimulation site resulted in a measurable membrane potential responses starting $10-15 \mathrm{~ms}$ after the onset of photostimulation with the peak of the response occurring at $26.8 \pm 5.5 \mathrm{~ms}$, reflecting the latency to induce action potentials in the presynaptic L4 cells, the presynaptic action potential conduction, synaptic release and transmission latencies, and propagation of the EPSP to the soma and tip of the patch-clamp electrode. In the example cell shown in Figure 4-4B, close examination of the membrane potential traces revealed clusters of overlapping EPSPs (Figure 4-5A).

Using individual EPSPs that were isolated and clearly separated (i.e. spontaneous or more than $200 \mathrm{~ms}$ after the onset of photostimulation), parameters of the individual EPSPs were measured. The peak amplitude of isolated EPSPs (from three cells, 15 


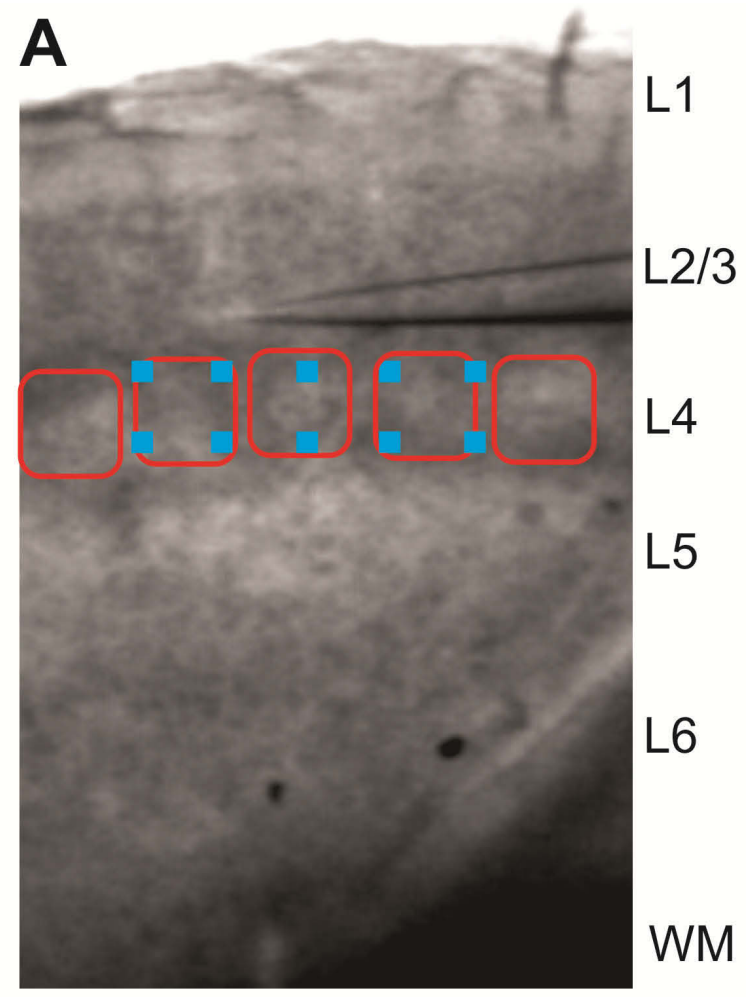

B

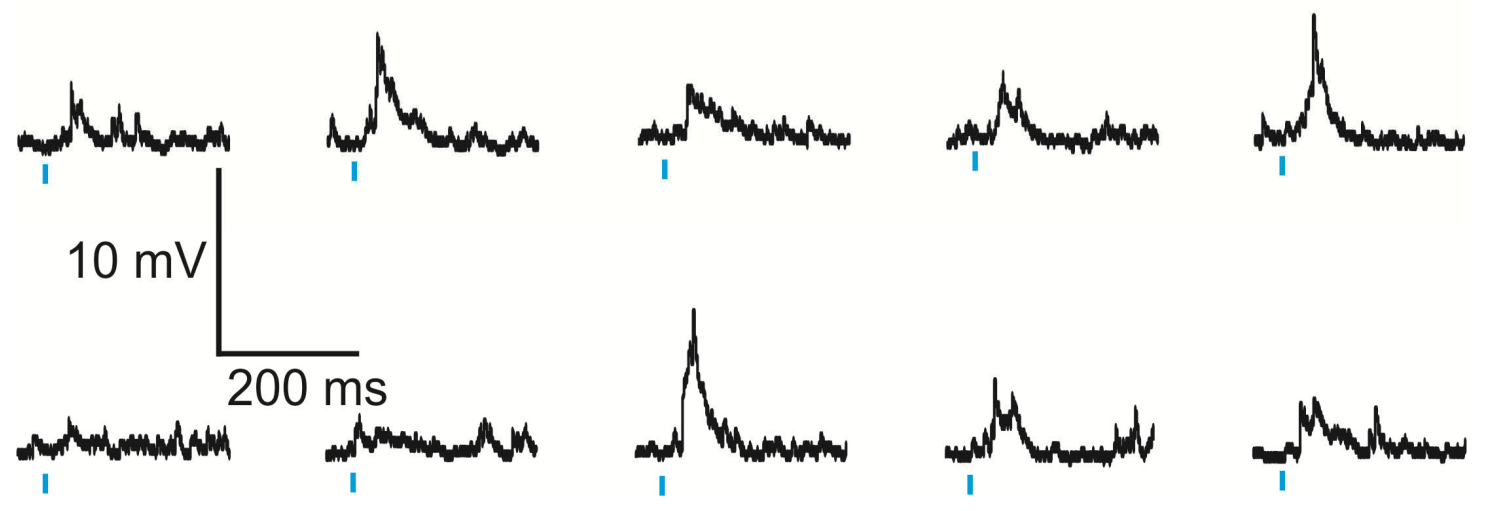

Figure 4-4. Presynaptic DLP photostimulation of neocortical L4 and the postsynaptic response in $\mathrm{L} 2 / 3$

(A) Coronal somatosensory barrel cortex slice in recording chamber with the position of photostimulation sites indicated by blue squares (confirmed with video frame freezes). The recording electrode is visible coming from the right, recording membrane potential from a pyramidal cell in L2/3. The approximate boundaries of L4 "barrels" are indicated by red squares $(150 \mu \mathrm{m} \times 150 \mu \mathrm{m})$. (B) Membrane potential responses to activation of each of the ten phostimulation sites. The timing of photostimuli are indicated with the blue dash below the trace. Each site was stimulated individually with a 30 -second recovery period in between photostimuli. 
A L2/3 - membrane potential
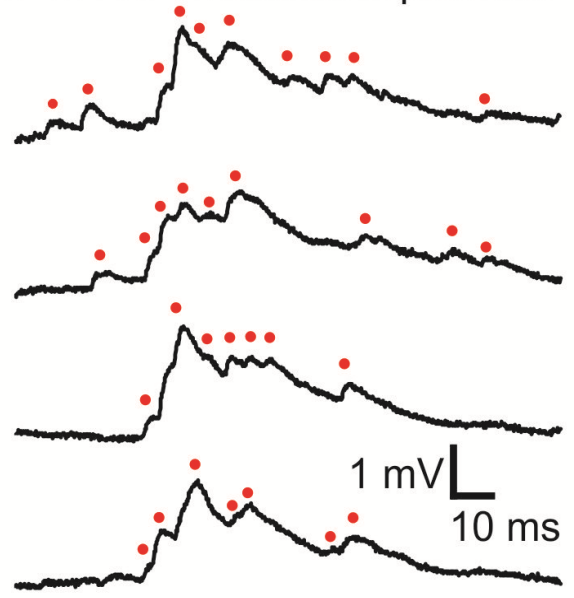

B L4 - action potentials
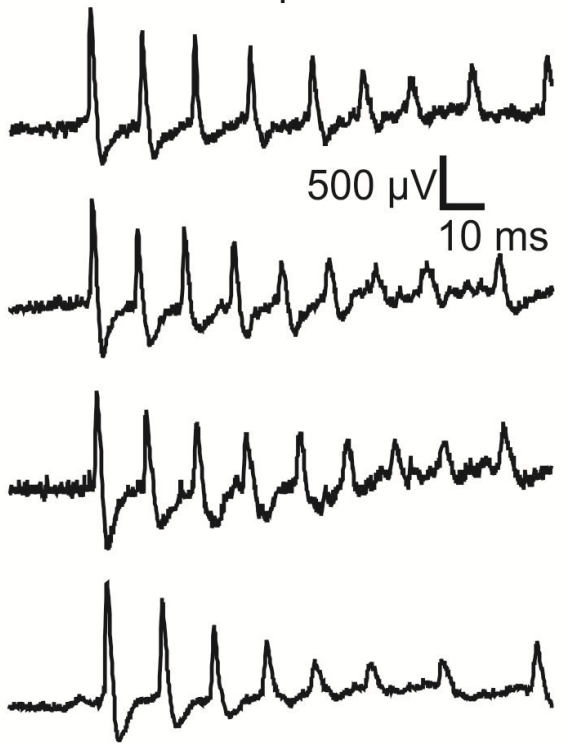

Figure 4-5. Pre- and post-synaptic response to L4 photostimulation

(A) Postsynaptic membrane potential responses to photostimulation of a single site in L4. Four trials are shown, with each trial exhibiting multiple overlapping EPSPs, which are indicated by red dots. (B) On-cell recordings revealing the action-potential output of a presynaptic L4 cell. Four trials in response to activation of the stimulation site closest to the soma of the targeted cell are shown. The time windows relative to photostimulation are identical in the top and bottom set of traces. Variability in the precise timing of presynaptic action potential output is probably the cause of variability in the precise timing of EPSPs in the postsynaptic response. 
EPSPs each, $\mathrm{n}=45$ ) was $0.66 \pm 0.32 \mathrm{mV}$, a $20-80 \%$ rise time of $1.01 \pm 0.66 \mathrm{~ms}$, a $80-20 \%$ decay time of $4.94 \pm 2.23 \mathrm{~ms}$, and an area under the curve of $3.2 \pm 0.9 \mathrm{~ms} * \mathrm{mV}$. These same EPSP parameters have been previously determined in rat with paired recordings of L2/3 pyramidal neurons and L4 spiny neurons [51] and are in close agreement. The advantage with this system or other photostimulation systems over paired recording techniques is that a large number of presynaptic sites and intermediate-range synaptic connectivity can be quickly and efficiently mapped in slices.

It was also important to characterize the presynaptic L4 action-potential output. To this end, we performed loose cell attached recordings of $\mathrm{L} 4$ cells $(\mathrm{n}=15)$ and photostimulated these cells with the same photostimulation pattern applied during L2/3 recordings (Figure 4-5B). These recordings thus reveal the presynaptic activity driving the responses observed in L2/3 neurons. More than half of cells in L4 $(n=8)$ responded to stimulation of one or more sites during the sequential stimulus sequence. In these cells, activation of the site with the strongest response induced a burst of action potentials with a maximum frequency of $143.8 \pm 63.4 \mathrm{~Hz}$ (mean \pm stdev). The first action potentials in these cells were induced $10-30 \mathrm{~ms}$ after the onset of photostimulation, and $55 \%$ of the spikes occurred within a window of $0-150 \mathrm{~ms}$ after the onset of site activation, with isolated action potentials occurring up to few seconds later. This envelope of action potential induction following photostimulation is identical to the window over which EPSPs arrive in L2/3 following photostimulation (Figure 4-5A). A detailed analysis of L4 responses to patterned photostimulation can be found in the section titled $\mathbf{L} 4$ Presynaptic Mechanisms of Supralinear Synaptic Summation.

Occasionally ( $\mathrm{n}=5$ out of 15$)$, L4 cells would respond to activation of multiple sites. In these cells, the sites inducing an action potential were more likely to be lateral (up to $350 \mu \mathrm{m}$ (4 sites) away) relative to the soma. Although some sites capable of inducing a response were deeper ( $86 \mu \mathrm{m}$ ( 1 site) away). A partial explanation for this is due to the lateral geometry of L4 dendrites. This, though, is unlikely because the asymmetrical L4 dendrites are oriented vertically, with lateral spans of less than $100 \mu \mathrm{m}$ [124] and pruned to be contained within a single barrel [14,125]. Lateral synaptic coupling of L4 cells has been investigated with geometric analysis of neuronal reconstructions in rat [43], which show geometric overlap of axonal and dendritic domains restricted to barrels. Physiological studies using paired recordings also report strong synaptic coupling with connections that were largely restricted to the same barrel $[14,124]$. Interbarrel connections, while rare, were reported to exist in these paired recordings. Paired recordings though, are inherently biased towards short-range and high-probability connections because short synaptic connections in slices are preferentially preserved during the slicing process. Our lateral connectivity data, though, was obtained from a technique that strongly activates several cells at each presynaptic site and more efficiently screens for long-range and low-probability connections. Strong lateral coupling of L4 cells, even between barrels, may still be possible and dispute the notion that each L4 barrel is a laterally-isolated excitatory network.

Action potentials in L4 cells were previously found to reliably induce EPSPs in synaptically coupled L2/3 cells with a failure rate of $\sim 5 \%$ in rat; [51]. These recordings 
revealed that a fast train of action potentials in a single synaptically-coupled L4 cell results in a reliable constant-amplitude (or slightly adapting, depending on the frequency) train of nonoverlapping EPSPs in the postsynaptic L2/3 cell [51]. The responses in Figure 4-5A and responses from all other cells in the exclusion criteria, however, show a tightly packed ensemble of EPSPs of varying amplitudes. This suggests that L4 photostimulation activates multiple presynaptic L4 cells within each site and that converging input from L4 onto L2/3 results in this typical membrane potential response.

To obtain a rough estimate of the number of synaptically coupled cells stimulated per stimulation site, we calculated the average area under the compound EPSP (from the onset of photostimulation to $150 \mathrm{~ms}$ after the onset) of the strongest L2/3 response $(94.5$ $\left.\mathrm{ms}^{*} \mathrm{mV}, \mathrm{A}_{\text {response }}\right)$ and divided by the product of the average area under the curve of the isolated EPSPs $\left(3.2 \mathrm{~ms} * \mathrm{mV}, \mathrm{A}_{\text {epsp }}\right)$ and the number of EPSPs $\left(\mathrm{n}_{\mathrm{epsp}}\right)$ that can be expected when each presynaptic neuron fires a single action potential (maximum case) or each presynaptic neuron fires a $150-\mathrm{Hz}$ burst of action potentials (minimum case). We also allowed for a failure rate in the maximum estimate. The following equation was used to calculate the values tabulated in Table 4-1:

$$
\mathrm{n}_{\text {pre }}=\left[\mathrm{A}_{\text {response }} /\left(\mathrm{n}_{\text {epsp }} * \mathrm{~A}_{\mathrm{epsp}}\right)\right] *[1-(\text { failure rate })]
$$

These estimates are consistent with anatomical data. Based on neuronal density measurements in the mouse neocortex [126] of $9.2 \times 10^{4}$ neurons per $\mathrm{mm}^{3}$, or a maximum of $2.5 \times 10^{5}$ neurons per $\mathrm{mm}^{3}$ if the higher density of neurons in L4 of granular cortex is considered [127], there are 8.6-23.3 neurons underneath an 8 pixel x 8 pixel $(21.6 \mu \mathrm{m} x$ $21.6 \mu \mathrm{m}$ ) photostimulation site, assuming a $200-\mu \mathrm{m}$ thick core of healthy neurons in a $300-\mu \mathrm{m}$ thick slice. Due to light scatter and glutamate diffusion the physiologically effective size of a photostimulation site is closer to a cylinder with an estimated $43 \mu \mathrm{m}$ radius, leading to an estimated maximal number of 106.9-290.5 neurons per photostimulation site. The probability of synaptic connections between L4 and L2/3 neurons in acute slices was estimated to be $28 \%$ (connection ratio 1:3.6 in rat; [128], resulting in $2.4-81.3$ presynaptic L4 neurons per photostimulation site.

It may be possible to reduce the number of neurons activated at each site with techniques analogous to minimal photostimulation [129] by decreasing the dwell time of each photostimulation site or by adjusting the grayscale modulation of the mirrors in each site. Allowing for reasonable recovery times between trials (20s or more) these steps are prohibitively time-consuming for patch-clamp experiments with 10 sites but could be

Table 4-1. Maximum and minimum estimates of the number of neurons presynaptic to $\mathrm{L} 2 / 3$ stimulated by activation of single $\mathrm{L} 4$ sites

\begin{tabular}{|c|c|c|c|c|}
\hline Case & $\mathbf{n}_{\mathrm{AP}}$ & EPSP failure rate & $\mathbf{n}_{\text {EPSP }}$ & $\mathbf{n}_{\text {pre }}$ \\
\hline Minimum & $\begin{array}{l}30(150 \mathrm{~Hz} * 200 \\
\mathrm{ms})\end{array}$ & $0 \%$ & 30 & 0.98 \\
\hline Maximum & 1 & $10 \%$ & 0.9 & 32.81 \\
\hline
\end{tabular}


performed using sharp electrodes or perforated patch techniques. For example, if on average three trials of five dwell time settings are required to determine the minimal photostimulation settings for 10 sites, the time required is:

\section{0 sites $\times 5$ settings $\times 3$ trials $\times 20$ seconds $=50$ minutes}

A typical whole-cell recording will be good for between 30 and 60 minutes, leaving little or no time for an experiment after stimulus parameters are determined. Sharp or extracellular recordings can last a little longer, but have their own disadvantages and were not performed here.

These experiments demonstrate the utility of sequential photostimulation in characterizing L4 synaptic input to L2/3. These results are in close agreement with previous data obtained from paired recordings. Previous synaptic connectivity mapping experiments utilizing photostimulation techniques, however, were generally performed in voltage clamp, making it difficult to make a direct comparison to existing techniques. The voltage-clamp configuration allows investigators to isolate specific components of the synaptic currents (i.e. NMDA, AMPA, GABA, etc.) by clamping at the various reversal potentials. However, these experiments involved the integrative properties of neurons and were thus performed in whole-cell passive current-clamp mode.

\section{Timing-Dependent Nonlinear Temporal Summation}

The following set of experiments was designed to investigate the temporal synaptic integration of L 4 synaptic input to L2/3 of the whisker barrel cortex. A previously untestable hypothesis is that the summation of L4 synaptic input by L2/3 depends on the timing of presynaptic activity. In these experiments, we systematically investigate and quantify the timing dependence of summation of L4 input to L2/3. We further hypothesize that synchronous input sums more strongly than asynchronous input and that the summation is greater than would be expected from a linear integration of input. The only way of inducing action potentials with laminar specificity in anatomically distributed sites, and therefore testing this hypothesis, is by utilizing the parallel nature of the DLP photostimulation system.

This data set comes from the same set of cells as in the previous section, with strict exclusion criteria used to maximize cell health and physiological soundness of the results. Briefly, intracellular patch-clamp recordings in whole cell-mode were obtained from visually identified L2/3 pyramidal cells. Ten sites on an $86 \mu \mathrm{m}$ (32-pixel) grid within L4 were selected using the GUI (Figure 4-4A). The dwell time of sites was adjusted such that each site resulted in a subthreshold response, with the majority of dwell times falling between $2 \mathrm{~ms}$ and $10 \mathrm{~ms}$. Sites were typically binned in groups of $8 \mathrm{x}$ 8 pixels $(21.6 \mu \mathrm{m} \times 21.6 \mu \mathrm{m})$, although 4 x4-pixel groups $(10.8 \mu \mathrm{m} \times 10.8 \mu \mathrm{m})$ were also occasionally used when $2 \mathrm{~ms} 8 \times 8$ pixel sites induced suprathreshold responses by themselves. 
After the sequential activation of sites (as described in the previous section), the cells were allowed to recover for 30 seconds. For the next photostimulus, all ten sites were activated synchronously, with no latency between the onset times of sites. This stimulus pattern consisted of a single frame with all the bins of mirrors making up the sites turned to the "on" position. The frame was projected for 2-10 ms (depend ending on the dwell time) and then turned off. The resulting projection photolytically activated glutamate at all ten sites with perfect synchrony. In this case, there was no latency between the onset time of sites and the interstimulus interval (ISI) is assigned a value of 0 ms. After another 30-second recovery period, the temporal pattern of site activation was changed again; the first site was turned on $1 \mathrm{~ms}$ before the second, and then the third site $1 \mathrm{~ms}$ after that, and so on, resulting in a spatiotemporal pattern with an ISI of $1 \mathrm{~ms}$. The ISI was progressively increased. $2 \mathrm{~ms}$ between sites, $5 \mathrm{~ms}, 10 \mathrm{~ms}, 15 \mathrm{~ms}, 20 \mathrm{~ms}, 30 \mathrm{~ms}$, $40 \mathrm{~ms}, 50 \mathrm{~ms}$, and $100 \mathrm{~ms}$ were all tested during a recording. The dwell time remained constant, with temporal overlap of site activation occurring when the dwell time was greater than ISI. Between each sequence there was a recovery period of 30 seconds. Every cell within the exclusion criteria completely repolarized within a few seconds, with the additional recovery time empirically determined in preliminary experiments to be sufficient to avoid plasticity and any other short or long term changes in synaptic strength or integration. The complete sequence of the eleven ISIs was tested twice, further avoiding the confounding contribution of an order-dependent phenomenon. Additionally, input resistance and firing properties were sporadically monitored during the experiment, with significant rundown disqualifying a cell from analysis based on the exclusion criteria explicitly defined in Materials and Methods.

In all of the $L 2 / 3$ cells within the inclusion criteria $(n=20)$, temporal summation of L4 synaptic input resulted in suprathreshold membrane potential response to synchronized activation of all sites in L4 (ISI $=0 \mathrm{~ms}$, Example: Figure 4-6A). Desynchronized stimuli were less likely to result in suprathreshold responses (Example, Figure 4-6B). While some ( $\mathrm{n}=5$ out of 20 ) cells summed synaptic input to threshold in response to medium-latency stimuli (Interstimulus Intervals (ISIs) of $30 \mathrm{~ms}, 40 \mathrm{~ms}, 50$ $\mathrm{ms}$ ), no cells summed synaptic input to threshold when the stimuli had a long ISI (100 $\mathrm{ms}$ ), and most ( $\mathrm{n}=15$ out of 20 ) only reached threshold when stimulated with ISIs of 20 ms or less. The first and second trials recorded from each cell were similar in that the ISI threshold for spiking did not change between trials in most cells $(n=12$ out of 20$)$, changed by only one ISI (i.e. $20 \mathrm{~ms}$ to $30 \mathrm{~ms}$ ) in 3 cells, and two ISIs in the remaining 3 cells. A probability histogram illustrating the timing-dependence of surpathreshold synaptic integration is shown in Figure 4-6C.

The high-probability of suprathreshold response to low-latency stimuli was also reflected in the supralinearity of summation. Linearity was computed by dividing the area under the curve of the measured response by the area under the curve of the linear sum of responses to sequential stimulation in a measure formally called Summation Ratio (SR) (see Figure 4-7 and Materials and Methods for more details). SR values greater than unity reflect synaptic integration that is greater than that predicted by a linear model, and is therefore considered supralinear. For smaller ISIs ( $0 \mathrm{~ms}-20 \mathrm{~ms})$, the summation was highly supralinear $(\mathrm{SR}>10)$ for every cell within the exclusion criteria. In the majority 

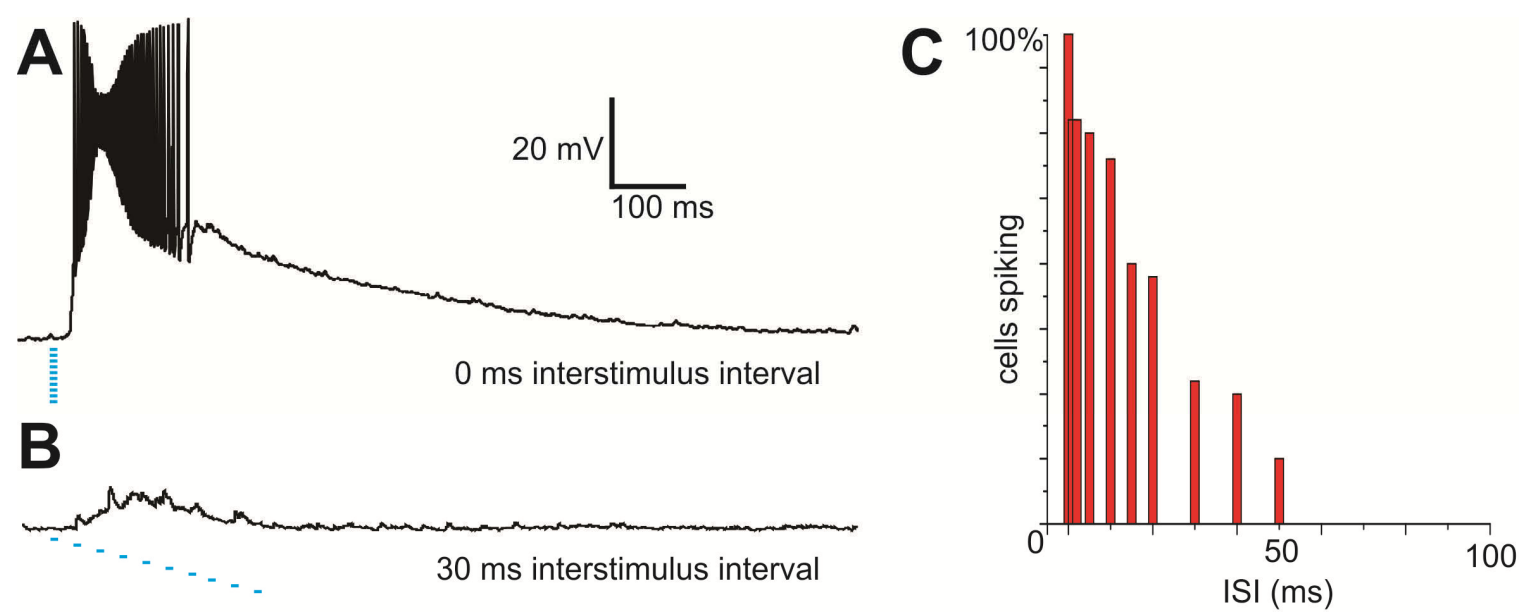

Figure 4-6. Timing-dependence of postsynaptic action potential induction in response to parallel photostimulation

(A) Membrane potential response of an example L2/3 cell to fully synchronous activation ( $0 \mathrm{~ms}$ ISI) of all ten L4 sites. (B) Membrane potential response of the same postsynaptic L2/3 cell to fast sequential activation ( $30 \mathrm{~ms} \mathrm{ISI)} \mathrm{of} \mathrm{the} \mathrm{photostimulation} \mathrm{sites.} \mathrm{In} \mathrm{both} \mathbf{A}$ and $\mathbf{B}$, the timing of site activation is indicated with blue dashes, with each dash representing the on time of a different site. (C) Probability histogram of trials (2 trials from each of 20 cells) in which the membrane potential reached threshold following parallel photostimulation as a function of ISI. 


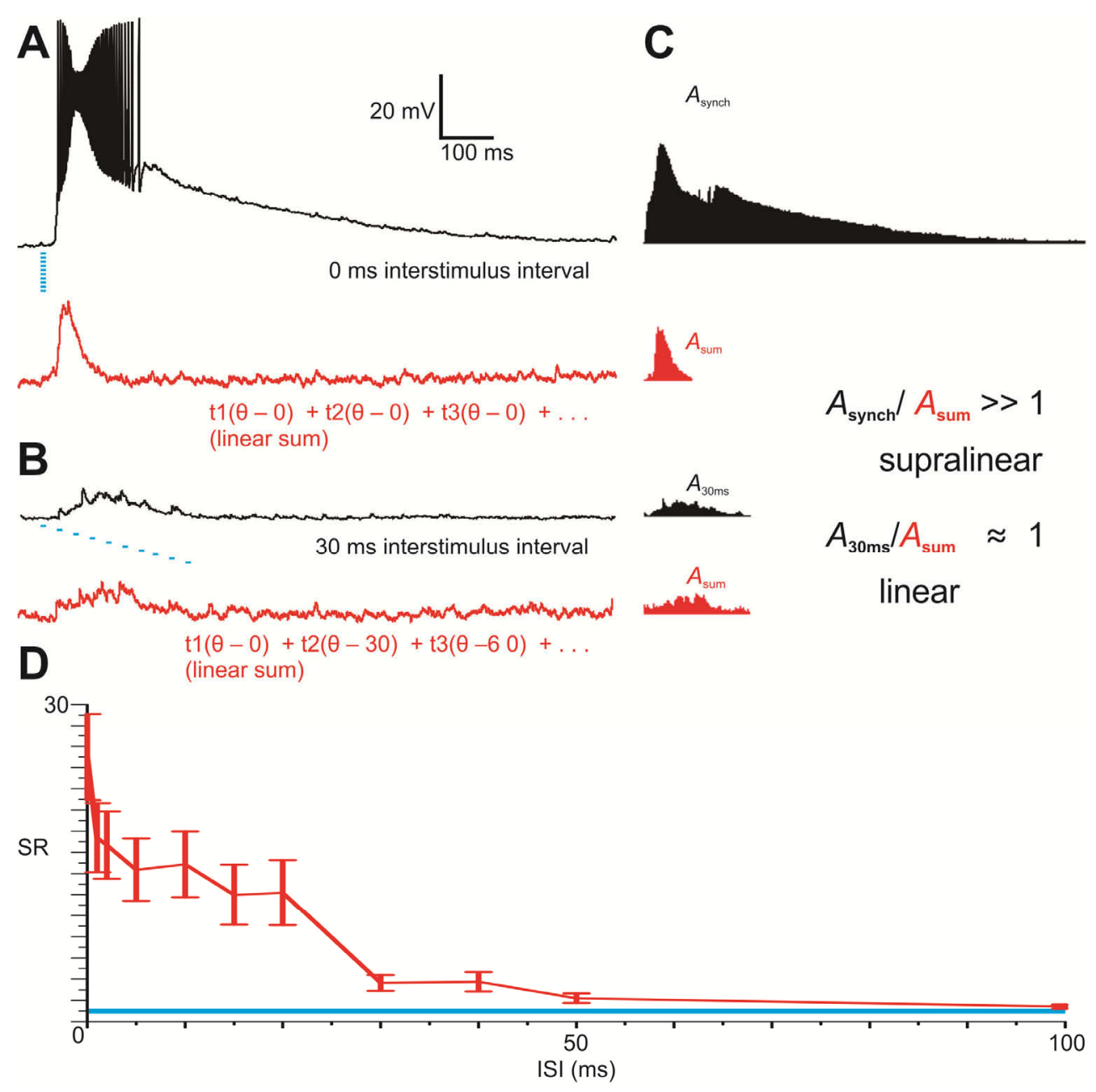

Figure 4-7. Calculation of Summation Ratio (SR) and timing-dependence of synaptic temporal summation.

(A) Membrane potential response of an example L2/3 cell to fully synchronous activation ( 0 ms ISI) of all ten L4 sites (black trace) and the linear sum of the individual sequential-site membrane potential responses (red trace, see Materials and Methods).

(B) Membrane potential response of the postsynaptic L2/3 cell to fast sequential activation (30 ms ISI) of the photostimulation sites (black trace) and the linear time-shifted sum of the individual sequential-site membrane potential responses (red trace, see Materials and Methods). In both $\mathbf{A}$ and $\mathbf{B}$, the timing of site activation is indicated with blue dashes, with each dash representing the on time of a different site. (C) Method for determining the supralinearity of temporal integration by calculation of SR. The shapes are graphical representations of the area under the curve of the corresponding traces on the left in A. Numerical area values (in $\mathrm{mV}^{*} \mathrm{~ms}$ ) are used to calculate the SR (i.e. Asynch/Asum). Values significantly greater than unity are considered supralinear. (D) SR plotted against ISI ( $n=20$ cells), illustrating the timing-dependence of synaptic non-linear summation. Error bars represent the standard error. The blue horizontal bar represents unity $(\mathrm{SR}=1)$. 
of cells, SR for ISIs $30 \mathrm{~ms}$ and greater were close to linear $(\mathrm{SR} \sim 1)$ or sublinear $(\mathrm{SR}<1)$. Four cells exhibited supralinear summation of synaptic inputs when the ISIs were between $30 \mathrm{~ms}$ and $50 \mathrm{~ms}$, but for every cell within the exclusion criteria, the SR was close to unity when the ISI was $100 \mathrm{~ms}$. Figure 4-7D plots the average SR versus ISI and exhibits a sharp inflection point between $20 \mathrm{~ms}$ and $30 \mathrm{~ms}$, representing the tendency of the L2/3 cells to integrate synaptic input supralinearly at ISIs below $30 \mathrm{~ms}$, and linearly or sublinearly at or above $30 \mathrm{~ms}$.

Together, these results support a timing-dependence on the supralinearity of input integration in the L4 to L2/3 connection, supporting the experimental hypothesis.

\section{L2/3 Postsynaptic Temporal Integration of L4 Synaptic Input}

The experiments described in the previous section establish a previously unreported phenomenon, but leave several open-ended questions. The mechanisms of the strong responses to synchronous photostimulation in the L4 to L2/3 are probably quite complex and potentially involve physiological mechanisms that are both pre- and post-synaptic. The following experiments are designed to test for a postsynaptic role of this circuit's sensitivity to stimulus timing.

We performed pharmacological manipulations of the postsynaptic cell with intracellular treatments. The pharmacological agents used in these experiments are membrane impermeable. When added to the intracellular electrode solution, the drugs diffuse into the cell but not anywhere else in the slice, effectively limiting the manipulation to the postsynaptic component of the circuit.

QX-314, a membrane impermeant lidocaine-derivative, when added to the electrode pipette solution, antagonizes voltage-gated ion channels within the target postsynaptic cell exclusively. Most-noticeably, the voltage-gated-sodium-channel-dependent action potential is strongly reduced in magnitude or completely abolished within minutes of obtaining an intracellular recording as QX-314 diffuses into the cell body and dendrites, but not outside the cell. Altering the intrinsic excitability of the postsynaptic L2/3 cell in this way does not eliminate the timing-dependence of synaptic temporal integration, but in fact enhances the selectivity for precisely-timed input as reflected in the increase in SR for low-latency L4 input (significantly different from control at ISIs of 0, 1, 5, 10, 15, $20 \mathrm{~ms}$ ) (Figure 4-8C), possibly due to the increased somatic input resistance and altered intrinsic excitability of QX-314-treated cells.

A concern with using the SR as a measure of linearity concerns the validity of applying this analysis to spiking cells, with spike-associated after depolarization and hyperpolarization potentials. Synaptic stimulation, especially with neurotransmitter uncaging, is rarely performed using passive whole-cell recordings, but to maintain physiological soundness in an investigation of synaptic integration, we felt like the resting membrane potential, after potentials, synaptic driving force, and all other factors 
Figure 4-8. Intracellular pharmacological manipulations of L2/3 pyramidal cells and the resultant electrophysiological responses to $\mathrm{L} 4$ photostimulation
(A) Examples of L2/3 electrophysiological responses to L4 photostimulation with the addition of $10 \mathrm{mM}$ EGTA (top) or $1 \mathrm{mM} \mathrm{QX-314} \mathrm{(middle)} \mathrm{to} \mathrm{the} \mathrm{standard} \mathrm{electrode}$ pipette solution. The timing of site activation is indicated beneath the control traces with purple dashes. Traces on the left are in response to a photostimulation pattern with an ISI of $0 \mathrm{~ms}$ and traces on the right are in response to a photostimulation pattern with an ISI $30 \mathrm{~ms}$. Two trials (overlapping traces) of each are shown. (B) Normalized spike count (count divided by maximum of count from all ISIs) for both manipulations as a function of ISI. (Control $n=20$, EGTA $n=4, Q X-314 n=4$, error bars report standard error) (C) SR for both manipulations as a function of ISI (control $n=20$, EGTA $n=4$, QX-314 $\mathrm{n}=4$, error bars report S.E.). The top and bottom panels show two different scales with the yellow box indicating the zoomed in portion. Orange asterisks indicate statistically significant differences (see Materials and Methods). 


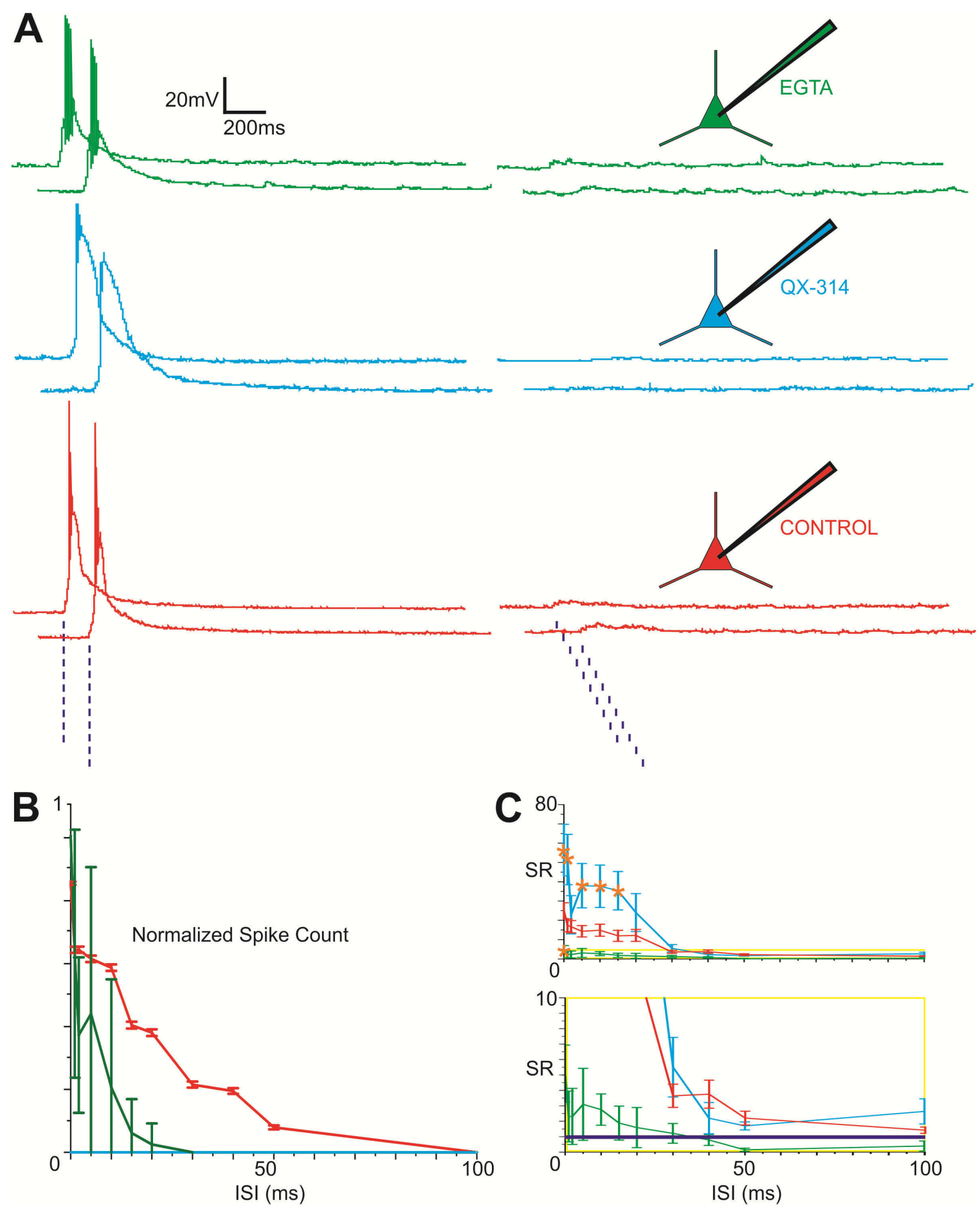


possible. Furthermore, the postsynaptic processing of inputs shouldn't be viewed as an artifact, but rather a physiologically-relevant contribution to the supralinearity of synaptic summation.

Regardless, we addressed this issue by blocking spikes in the postsynaptic cell. The first attempt of doing this, which was only performed a couple times, was to hyperpolarize the cell in an effort to minimize voltage-gated sodium channel activation. However, synchronized stimuli still drove the membrane potential to threshold. This is probably partially due to an increased excitatory synaptic driving force, but also potentially due to the extreme supralinearity of synaptic summation. Also, the limited dendritic spread of somatically-induced hyperpolarization, which would not successfully hyperpolarize distant cellular compartments, would not exclude the possibility that suprathreshold summation is compartmentalized to the distal dendrites. However, the pharmacological blockade of action potentials with QX-314 enhances the supralinearity of synaptic summation, the opposite of what one would expect if the supralinearity was simply due to the suprathreshold nature of the stimuli, which provides strong evidence that this is not the case.

EGTA, a calcium chealator, when added to the intracellular electrode solution, buffers intracellular calcium to a low concentration, preventing the accumulation of calcium influxed through NMDA receptors and voltage-gated calcium channels thereby reducing calcium-activated ion channels currents, such as BK, IK, and SK potassium currents. This treatment decreases the SR to near-linear levels for all ISIs (Figure 4-8C, significantly different from control at $0 \mathrm{~ms}$ ). However, the spike count vs. ISI plot (Figure 4-8B) still reflects a higher probability of spiking in response to precisely timed-stimuli. L2/3 action potentials were induced postsynaptically for ISIs of $20 \mathrm{~ms}$ or less in this treatment group.

The effect of these postsynaptic manipulations strongly suggests that postsynaptic processing of synaptic inputs contributes to the supralinearity of L4 synaptic summation by $\mathrm{L} 2 / 3$.

\section{L4 Presynaptic Mechanisms of Supralinear Synaptic Summation}

A presynaptic mechanism may also potentially contribute to the L2/3 suprathreshold response to low-latency stimuli, but not long-latency stimuli. In the previous section, presynaptic L4 sites were considered independent for analysis purposes; however, the lateral coupling of L4 cells may result in increased presynaptic firing rates with decreases in ISI. It is also possible that stimulus patterns with shorter ISIs activate a larger population of L4 cells. If either possibility were the case, the increases in L4 firing rates at lower ISIs would result in a predictable increase in the frequency of L $2 / 3$ input, which would predictably increase the SR.

On-cell measurements of L4 cells $(\mathrm{n}=15$ cells), which allow long-lasting

measurements of action potential output, were performed to test these hypotheses. These 
cells were stimulated with the same set of patterns as in the previous section; therefore these recordings represent the presynaptic L4 activity driving the postsynaptic response in $\mathrm{L} 2 / 3$ described in the previous section. These pairs of cells were targeted within the same field of view $(<100 \mu \mathrm{m}$ separation) and always within a single barrel.

Every L4 cell responded to at least one of the patterned photostimulation patterns. However, only 8 of 15 L4 cells from which recordings were obtained responded to activation of any single site during the sequential stimulation. This suggests that additional L4 cells are activated during patterned stimuli and may contribute to the supralinearity of synaptic summation performed by L2/3 cells during patterned stimuli.

The average L4 firing rate and total number of action potentials following photostimulation was only weakly modulated by ISI. Action potentials occurring during the integration window (see Materials and Methods) were counted for every ISI and averaged across cells (Figure 4-9A). This plot exhibits a downward trend, but many individual L4 cells did not exhibit this trend (Figure 4-9B). The maximum response from 2 of the 15 cells occurred in response to the most desynchronized stimulation pattern (ISI of $100 \mathrm{~ms}$ ) and in response to intermediate ISIs for 4 of the 15 cells (ISIs of 20, 30, and $40 \mathrm{~ms}$ ). This modulation of spike counts with ISI may suggest that increased presynaptic L4 firing during synchronized stimuli increases synaptic input to L2/3, resulting in a supralinear summation of low-latency stimulation, although the existence of L4 cells that more strongly response to long-latency stimuli confound the interpretation of this result.

The timing of action-potential output was very strongly modulated by ISI (Figure 4-9C). For ISIs between $0 \mathrm{~ms}$ and $10 \mathrm{~ms}$, the first induced action potential occurred very reliably within a window that was $10-50 \mathrm{~ms}$ after the onset of stimulation. With each successive increase in ISI, the first action potential was induced later and with less predictable timing, as indicated by the increasing standard errors in the plot.

Additionally, a few $(n=4)$ paired on-cell recordings were performed with a goal of determining if a temporal relationships exists between cells that fire during the same photostimulation period. In all four pairs, both cells fired action potentials in response to ISIs of 0,1 , and $2 \mathrm{~ms}$. In two pairs, both cells fired action potentials up to $20 \mathrm{~ms}$. Meaningful quantification of the temporal relationship of L4 input will probably require the simultaneous measurement of a large number of cells, which is difficult or impossible with traditional electrophysiology techniques. However, optical recordings of activity, using voltage sensitive dyes and calcium indicators, is a planned as a follow up experiment and will allow us to simultaneously record action potentials from a large number of cells and more thoroughly characterize the temporal characteristics of L4 presynaptic action-potential output in response to patterned photostimulation.

The weak modulation of action potential output with regard to ISI fails to refute the hypothesis stated in this section. A presynaptic mechanism within L4 could be contributing to the supralinear summation of low-latency photostimuli. 
A

Average Frequency

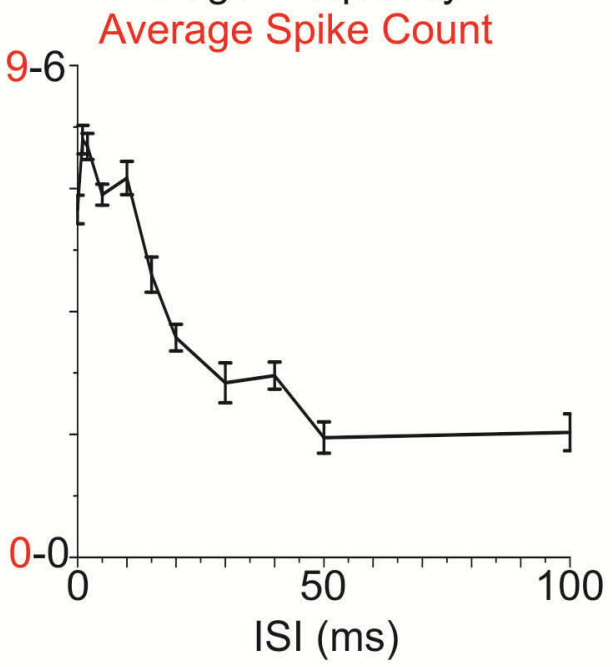

B
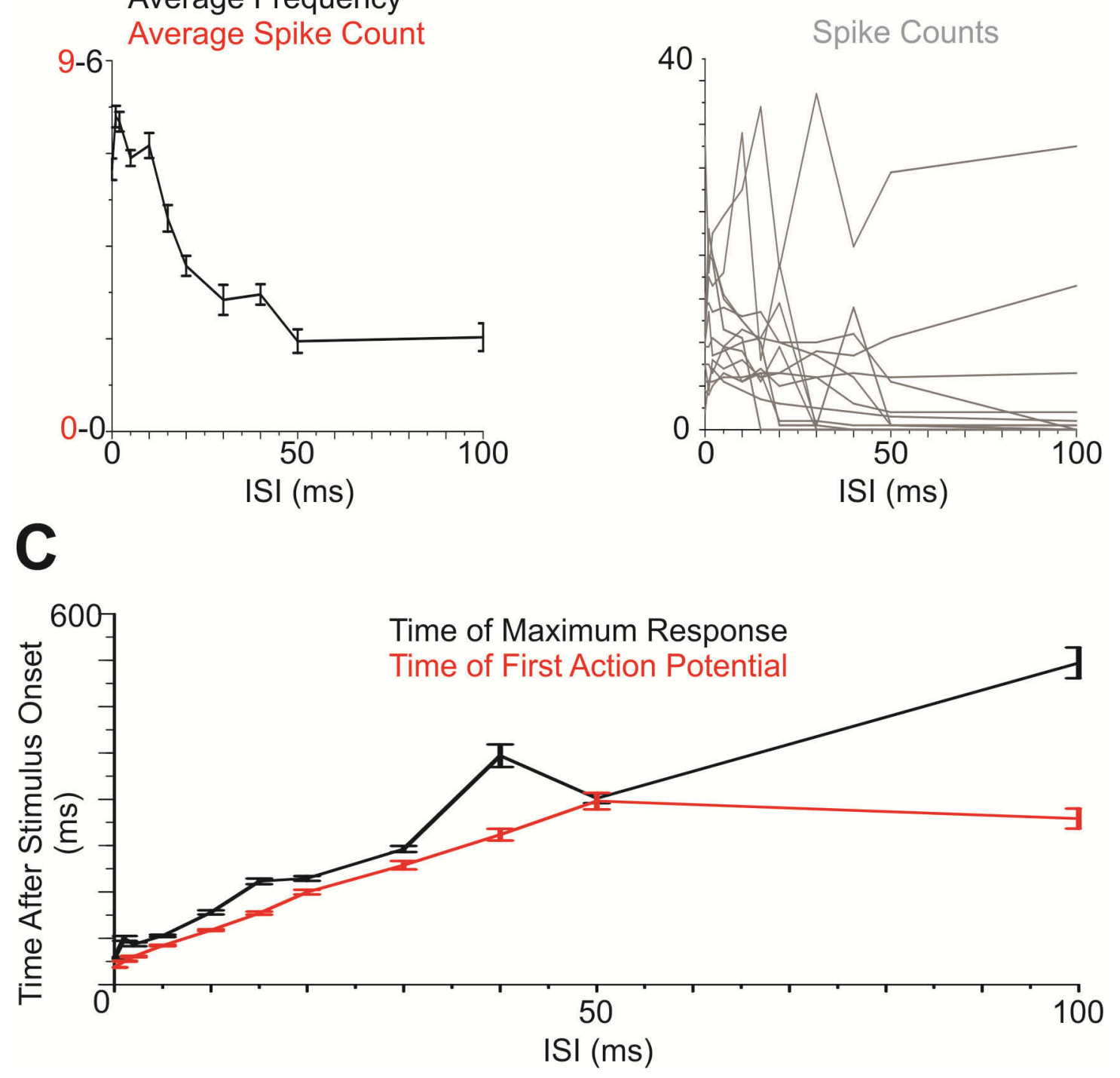

Figure 4-9. Quantification of L4 action-potential output

(A) Average magnitude of the action-potential output of L4 cells during the $0 \mathrm{~ms}$ to1500 ms window following photostimulation $(\mathrm{n}=15)$. (B) Same plot as in A, with spike counts by cell, illustrating the weak modulation of action-potential output by ISI in some cells, with some cells ( $\mathrm{n}=6$ of 15) exhibiting a trend opposite of the average. (C) Timing of the first action potential and period of maximum response relative to the onset of photostimulation $(\mathrm{n}=15)$. 

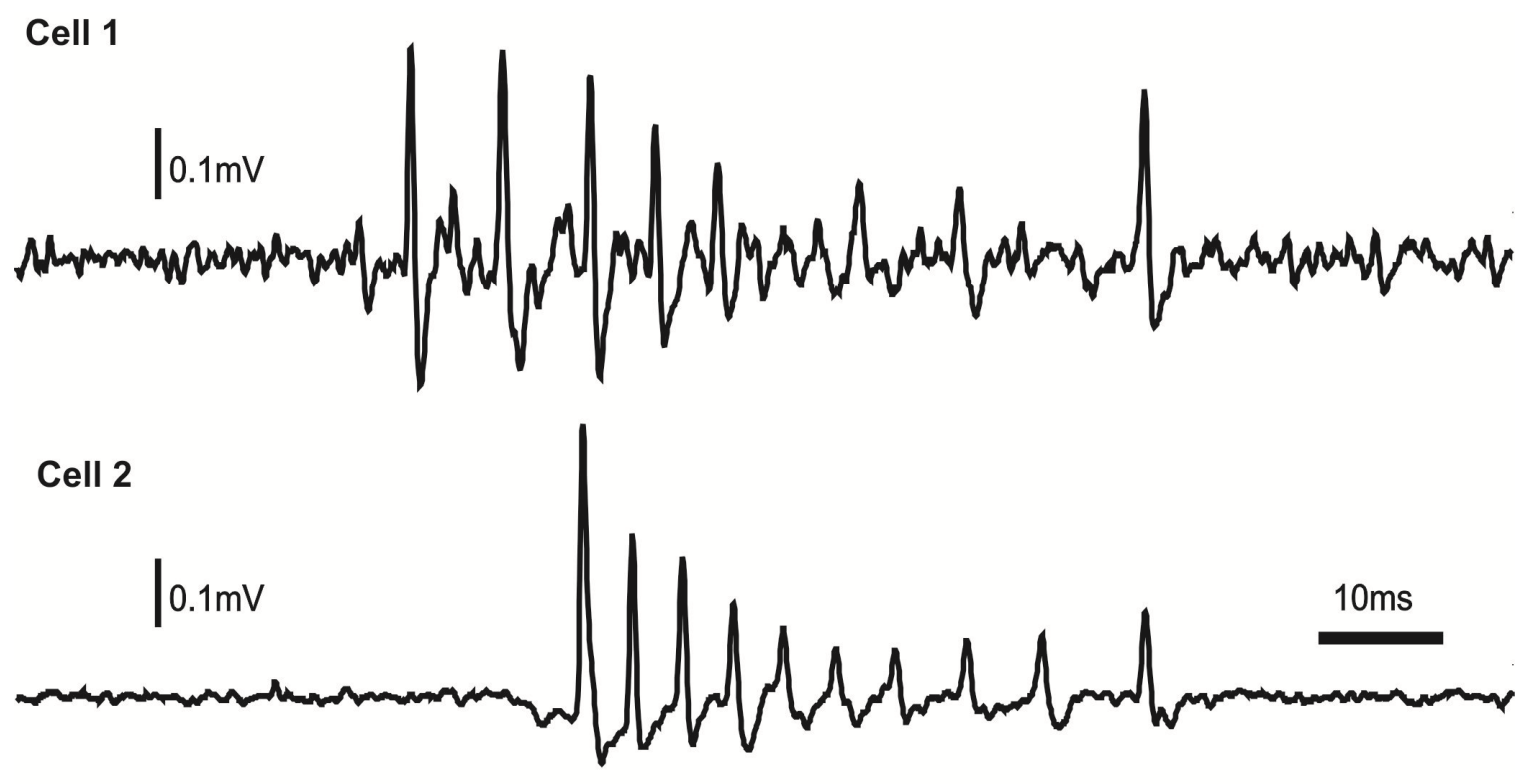

Figure 4-10. Example paired on-cell L4 recording in response to photostimulation with an ISI of 0 ms

Simultaneous on-cell recordings of two L4 cells. The blue dash indicates the timing of the synchronous photostimulation pattern. 


\section{AMPA Dependence and NMDA Modulation of Temporal Synaptic Integration}

Network-wide pharmacological manipulations of the slice bathing solution were also performed, with experiments investigating the effects of blocking the NMDA and AMPA components of EPSPs. These experiments were designed to test the hypothesis that the fast component of excitatory synaptic transmission (primarily gated by AMPA receptors) is necessary for the timing-dependence of nonlinear synaptic integration to occur. NMDA receptors were hypothesized to modulate the timing dependence by lengthening the decay time of EPSPs and therefore widening the temporal integration window of the $\mathrm{L} 2 / 3$ cells.

In these experiments, four of the healthiest control cells were selected for additional manipulations after the conclusion of the first two rounds of photostimulation. NBQX, an AMPA receptor antagonist, was added to the bathing solution after the second round. The drug was allow 2 minutes to recirculate, during which the cells slightly hyperpolarized ( 1 to $4 \mathrm{mV}$ ), presumably due to a blockade of AMPA conductances activated by background activity or nonsynaptic events. These cells continued to be subject to the strict exclusion criteria and were only included in the data set if the same standard of cell health and excitability were maintained for two more rounds of photostimulation.

In these cells, the addition of NBQX completely blocked the response to sequential or patterned photostimulation, reflecting the dependence of intracortical synaptic excitation on the AMPA component of EPSPs. Photostimulation of L4 failed to drive L2/3 to threshold at any ISI (Figure 4-11D, Figure 4-11E) and the SR curve flattened to linear (SR 1) for all ISIs (Figure 4-11F). The cells within the exclusion criteria continued to fire overshooting action-potentials in response to current injection, eliminating the possibility that cells were no longer capable of reaching threshold. The significance of this manipulation was tested by using the previous two rounds of stimuli as an in-cell control, and significant differences from control were observed at ISIs of $0,1,2,5,10,15,20 \mathrm{~ms}$. The absence of significance at longer ISIs reflect the small response of all $\mathrm{L} 2 / 3$ cells in response to desynchronized photostimuli.

Similar to the experiments with NBQX, AP-5, an NMDA receptor antagonist, was added to the recirculating aCSF following two rounds of photostimulation. NBQX blocks the slow component of the EPSP and as expected, narrows the temporal integration windows of the L4 to L2/3 excitatory connection (Figure 4-11A). This is reflected in the normalized spike count, with the addition of AP-5 shifting the curve left, towards lower ISIs (Figure 4-11B), and also in the SR plot, which now shows supralinear summation for only the smallest ISIs $(<10 \mathrm{~ms}$, compared to $<30 \mathrm{~ms}$ before AP-5, Figure 4-11C, significantly different from control at ISIs of 2, 5, 10, 15, $20 \mathrm{~ms}$ ). 
Figure 4-11. Extracellular pharmacological manipulations of network-wide glutamate-gated currents and the resultant $L 2 / 3$ electrophysiological responses to L4 photostimulation
(A) Membrane potential responses to $0 \mathrm{~ms}, 5 \mathrm{~ms}, 10 \mathrm{~ms}, 15 \mathrm{~ms}$, and $20 \mathrm{~ms}$ ISIs before (left, black) and after (right, red) the addition of $50 \mu \mathrm{M}$ AP-5. The blue triangle beneath each trace indicates the onset of the photostimulation pattern. (B) Normalized spike count (count divided by maximum count from all ISIs) before (black) and after (red) the addition of AP-5 as a function of ISI. (C) SR before (black) and after (red) the addition of AP-5 as a function of ISI (D) Membrane potential responses to $0 \mathrm{~ms}, 5 \mathrm{~ms}, 10 \mathrm{~ms}, 15 \mathrm{~ms}$, and $20 \mathrm{~ms}$ ISIs before (left, black) and after (right, red) the addition of $10 \mu \mathrm{M}$ NBQX. (E) Normalized spike count (count divided by maximum of count from all ISIs) before (black) and after (red) the addition of NBQX as a function of ISI. (F) SR before (black) and after (red) the addition of NBQX as a function of ISI. Orange asterisks indicate statistically significant differences (see Materials and Methods). 

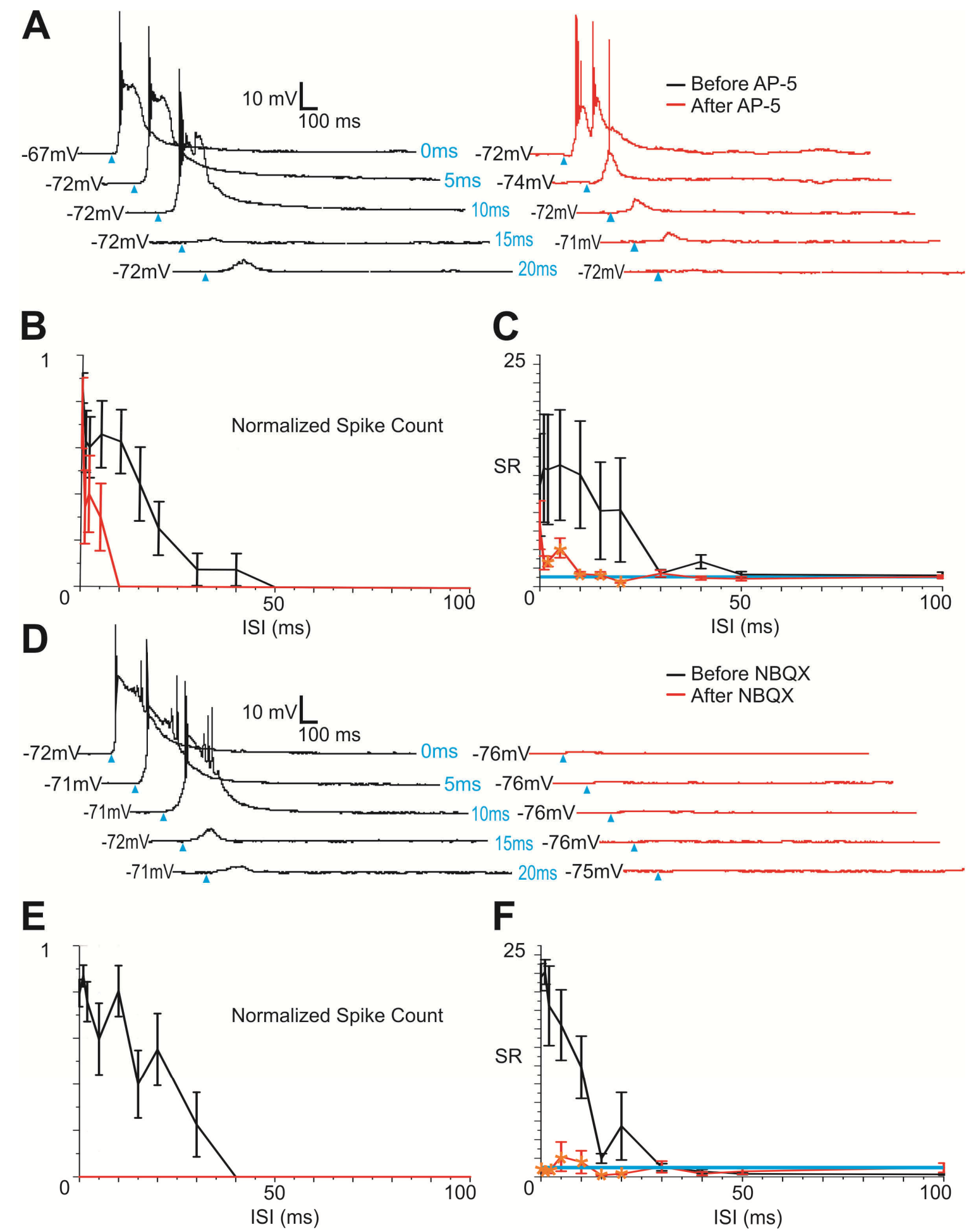


\section{Anatomical Arrangement and Temporal Order of Stimulus Sites}

As stated in previous sections, the spatial arrangement of stimulation sites remained fixed, with ten sites arranged on an $86 \mu \mathrm{m}$ grid (Figure 4-4A). The spatial arrangement of sites was always 5 sites wide and 2 sites tall. The anatomical arrangement of stimulus sites was verified with either post-hoc analysis of videos recording the photostimuli or live viewing of the photostimulation patterns on the microscope's video monitor.

L4 in mouse is approximately $150 \mu \mathrm{m}$ thick and each barrel is about $100 \mu \mathrm{m}$ wide. Vertically, the sites were always centered in L4 and precise vertical adjustments of the stimuli were often necessary. When centered as in the set of cells satisfying the exclusion criteria, the stimuli had approximately 20-50 $\mu \mathrm{m}$ of L4 on either side (deep and superficial) of the vertical extent of the ten sites (Figure 4-4A).

The radius of curvature of the barrel cortex is relatively large relative to the width of the stimulus pattern $(344 \mu \mathrm{m})$ (Figure 4-4A), and thus can be disregarded with respect to the anatomical arrangement of sites. The horizontal dimension of the stimulation sites extended across three adjacent barrels. Each barrel contained either two or four sites, depending on the precise position of the horizontal center.

The order of site activation was always fixed. The top-right (superficial-lateral) site was activated first followed by the bottom left (deep-lateral). The order then preceded medial. Because of this, the barrels were always stimulated lateral to medial, with the superficial portion of the barrel being stimulated before the deep portion. For barrels containing 2 sites, the barrel was stimulated over a window equal to two times the ISI and for a barrel containing 4 sites, the barrel was stimulated over a window equal to four times the ISI.

Another consequence of this fixed order of site activation was that the order of barrel activation was also fixed. The L2/3 cell from which postsynaptic responses were obtained was always oriented over the middle of the three barrels. The order of barrel activation was first: adjacent lateral, second: Principal (middle) and third: adjacent medial.

Interestingly, the strength of responses seemed qualitatively unmodulated by distance from the postsynaptic L2/3 cell. In Figure 4-4B, the spatial arrangement of L2/3 membrane potential responses match the spatial arrangement of photostimulation sites in Figure 4-4A. The expectation was that the closest site to the postsynaptic target is most strongly activated, but this was rarely the case, as exemplified by Figure 4-4. The reasons for this are unclear, but are potentially due to the strong lateral coupling of L4 barrels observed in these experiments. It is also possible that the L4 to L2/3 connection may be more laterally spread than previously reported. Synaptic couplings between pairs of intracellular recordings become increasingly improbable with increasing distance, introducing a sampling bias for high-probability high-reliability synaptic coupling. Extracellular recordings on the other hand are insensitive to non-spiking cells and are 
biased against rarely-spiking cells. Furthermore, extracellular recordings only report suprathreshold activity. Long-distance intracortical subthreshold connectivity may in fact more prevalent than previously thought, and revealed only in experiments not biased against weak, sparse, or unreliable connections between rarely-spiking cells. 


\section{CHAPTER 5. DISCUSSION}

\section{Interlaminar Cortical Processing of Sensory Input}

Specifying the timing of glutamate uncaging in L4 while measuring the electrophysiological response in postsynaptic L2/3 neurons allowed what we believe is the first investigation of interlaminar temporal action-potential propagation and synaptic integration in the neocortex. The DLP photostimulation technique applied in these experiments allow us to report a previously untestable phenomenon in a neocortical circuit, but may also prove instrumental in the investigation of other complexly-interconnected and dense microcircuits in the brain.

Within the whisker barrel cortex, projections from L4 to L2/3 are known to be one of the strongest interlaminar pathways [130,131] with L2/3 neurons receiving converging inputs from approximately 300-400 spiny L4 neurons [43]. Modeling studies based on experimentally-determined synaptic parameters [51] and the known in vivo firing probability of L4 neurons after whisker deflection [12] suggest that the peak membrane potential deflection resulting from the compound EPSPs is not sufficient to drive L2/3 pyramidal neurons to fire spikes. However, L2/3 pyramidal cell spike firing in response to whisker stimulation is well documented (ie. [11,39,46,132-135]), although with lower probabilities than that seen in L4 whisker-responsive cells. The authors of the modeling study acknowledge this discrepancy and suggest several possible solutions, one of which is the existence of spiking synchrony or temporal correlation in L4 which would drive L2/3 neurons with greater strength [52] as is the case with synchronously firing thalamic neurons driving L4 [136]. However, the presynaptic activity patterns preceding L2/3 spiking are unknown, thus the in vivo physiology and synaptic integration of this specific connection continues to elude characterization. Our experiments support the hypothesis that presynaptic synchrony more strongly drives L $2 / 3$ by showing that synchronous stimulation (i.e. low ISIs) of L4 neurons is much more likely to drive L2/3 pyramidal neurons to fire action potentials.

Experiments manipulating the internal pharmacology of the patch-clamped neurons were designed to determine if a mechanism within the postsynaptic L2/3 cell was responsible for the stronger propagation of precisely-timed L4 input, or if an emergent network or circuit property was responsible. Layer V pyramidal neurons exhibit a supralinear summation of synchronous or low-latency synaptic inputs. This phenomenon depends on dendritic conductances that are blocked by intracellular QX-314 [137]. L2/3

Adapted with permission.

5 Jerome J, Foehring RC, Armstrong WE, Spain WJ, Heck DH (2011) Parallel Optical Control of Spatiotemporal Neuronal Spike Activity Using High-Speed Digital Light Processing. Front Syst Neurosci 5. doi:10.3389/fnsys.2011.00070.

6 Jerome J, Heck DH (2011) The age of enlightenment: evolving opportunities in brain research through optical manipulation of neuronal activity. Front Syst Neurosci 5: 95. doi:10.3389/fnsys.2011.00095. 
pyramidal dendrites, while not as easily studied due to their smaller size, are known to contain ensembles of conductances that sustain regenerative events, such as back-propagating action potentials, dendritic spikes, and calcium transients [138-140]. Furthermore, the relative compactness and high morphological variability of L2/3 dendrites may have implications in the temporal synaptic integration of these cells [141$145]$.

These experiments, with QX-314 or EGTA added to the intracellular electrode solution, support a role of postsynaptic dendritic processing in the observed L2/3 response. It should be noted that the efficacy of intracellular treatments is not perfectly uniform due to limited diffusion into the distal dendrites, and that mechanisms compartmentalized to the distal dendrites, such as dendritic-spike initiation in the apical tuft of L2/3 neurons, may still be contributing to the timing-dependent temporal summation of L4 input in these cells. Imaging with voltage-sensitive dyes and other intracellular indicators with a high-speed CCD camera may be more able to elucidate the role of postsynaptic processing of spatiotemporally complex input and is a planned follow-up investigation. However, the statistical significance of these intracellular manipulations does clearly demonstrate a role of postsynaptic processing on the nonlinear timing-dependence of synaptic integration of L4 input to L2/3.

The spatiotemporal propagation of activity within large neuronal networks is a key component of the mechanisms of information processing and representation within the neocortex. A traditional view is that neurons carry information from one region to the next in a coding scheme based on firing rates [3,146,147]. In this scheme, increased firing in a presynaptic neuronal population results in a subsequent firing rate increase in the postsynaptic population. This scheme is supported by observations of highly variable firing rates in neurons and the observation that in sensory neurons, the strength of a stimulus is correlated with firing rate [1]. However, this coding scheme, if used exclusively in the central nervous system would require long integration times to conserve a firing rate code over multiple connections, making this coding potentially slow and inefficient $[4,6,148-156]$.

Other experiments and modeling studies support a coding scheme based on spike timing and synchrony [41]. In this mode of coding, synchronous activity in the presynaptic pool leads to a reliable and fast feedforward transmission to the postsynaptic pool, which in turn fires synchronously and transmits to the next pool in the chain. This coding scheme is supported by the finding that neurons in the neocortex receive synaptic input from multiple presynaptic cells, have short EPSP decay times, and data from the psychophysical studies supporting very fast visual processing times [157-160], which would necessitate a coding scheme that did not depend on averaging firing rate over a period of time. Sensory input increases the synchrony of functionally related central neurons in many sensory processing regions [5,152,161-163]. Additionally, multiple elegant investigations into single-cell physiology show a selective gating and higher action potential coupling to synchronous input [5,122,164-171]. If used, this coding scheme would allow for very fast processing times, high reliability, and high robustness. The advantages, disadvantages, and experimental support of rate coding and timing 
coding are reviewed in $[41,42]$. These two coding schemes are not necessarily mutually exclusive and may operate in parallel to code for different features of sensory input.

In modeling studies, the reliability and stability of rate coding and timing coding have been supported and debated, with parameters such as background noise, balance and timing of inhibition and excitation, and synaptic mode (conductance versus current) affecting the stability and likeliness of these two coding schemes $[53-58,62,63,147-$ 150,153,156,172-178]. However, technical limitations have restricted the feasibility of similar experiments in vivo or in vitro, which would require the simultaneous monitoring or manipulation of membrane potential in hundreds of cells simultaneously. This new massively parallel photostimulation technique allows an investigator to induce action potentials at hundreds of anatomically and spatially distinct locations with any temporal pattern, opening up the possibility for investigating the role of synchrony and timing of action potentials in activity propagation.

Results from these experiments support a stable propagation of synchrony, with postsynaptic spike probabilities and SR highest when presynaptic stimuli are synchronized. However, in our experiments, activity in L4 is still reliably propagated to L2/3 when stimuli are desynchronized to a 20 or $30 \mathrm{~ms}$ ISI. With ten sites and a $20 \mathrm{~ms}$ ISI, the activity in L4 occurs within a time window of $200 \mathrm{~ms}$, whereas most groups within modeled synfire chains fire within a window of $5 \mathrm{~ms}$ or less. Stability and reliably of action potential propagation and timing, then, may be less dependent on synchrony than expected from modeling studies. Recently, an optical recording technique has revealed that in slices, the temporal correlations between spiking neurons decrease as activity propagate from L4 to L2/3, calling into question the likeliness of a stably propagating synfire chain [177].

Nonlinear intracortical temporal synaptic integration may play a direct role in the processing of whisker input. While classical in vivo experiments attempted to elucidate the response properties and receptive fields of single-whisker stimuli [9-17], recent studies have suggested that simultaneous or low-latency input from multiple adjacent whiskers have significantly different neuronal responses [44-49]. Actively exploring mice use multiple whiskers simultaneously for object localization and identification and depend on this sensory input to navigate their environment and find food.

Hirata and Castro-Alamancos [44] performed a very elegant experiment in which they measured single-unit extracellular action potentials in L4 and L2/3 and additionally L2/3 intracellular membrane potential with in vivo sharp electrode recordings. Synchronous or low latency multiple-whisker stimulation $(<15 \mathrm{~ms}$ between whisker deflections), as compared to single-whisker stimulation, enhanced the precision and latency of spikes in L4. Higher precision L4 spiking is accompanied by an increased probability of suprathreshold activity in L2/3, representing an overall enhancement of responses to multi-whisker stimulation. However, with long latency stimulation (16 ms $50 \mathrm{~ms}$ between whisker deflections), the precision of L4 spikes and probability of L2/3 spikes are suppressed relative to responses from single-whisker stimulation. The intracellular recordings from L2/3 suggest temporal summation of precisely-timed 
convergent synaptic input is responsible for the $\mathrm{L} 2 / 3$ enhancement in response to low-latency stimuli. In our experiments, we systematically tested the response to different latencies of multiple-barrel stimulation. The large stimulation area possible with DLP photostimulation allowed us to stimulate the principal and adjacent barrels with precisely modulated latencies. Interestingly, the latency supralinearity threshold in our experiments (10 ms - $40 \mathrm{~ms}$ ) corresponded closely with the categorization of long- and short latency stimuli in the analysis by Hirata and Castro-Alamancos. Cells from our data set had thresholds that varied between $10 \mathrm{~ms}$ and $40 \mathrm{~ms}$, a precision that is possibly obscured by the pooling of data into two categories by Hirata and Castro-Alamancos [44].

Two older studies in rat report only suppressed or sublinear responses to multiple whisker stimulation, regardless of the latency [46,49]. Another study in rat, however, showed enhanced and even supralinear responses to multiple-whisker stimulation [48]. Discrepancies in these findings may be possibly explained by differences between species, animal age, depth or method of anesthesia, or other undocumented technical factors. Additionally, recordings from references cited in this paragraph were not layer-specific and are possibly the result of differential suppression/enhancement of specific layers during multi-whisker activity. Our experiments in the acute slice preparation allow us to specify the layers of stimulus and target layers for measuring a response. Planned experiments utilizing voltage-sensitive dyes in this preparation will allow us to measure responses in multiple layers simultaneously, as well as determine the time-course and propagation of excitatory intracortical activity.

\section{Digital Parallel Photostimulation}

The use of light for the manipulation of neuronal activity through photolytic release of neurotransmitters has opened up new vistas in experimental neuroscience due to the enhanced flexibility and specificity over electrical stimulation techniques. To take full advantage of these new experimental tools, we have developed a parallel light stimulation technology that allows emulation of the complexity of in vivo spatiotemporal neuronal activity patterns under in vitro experimental conditions.

The parallel photostimulation system described here allows precise spatiotemporal manipulation of action potential firing and subthreshold neuronal activity at a broad range of anatomical scales. Because the system's $>780,000$ light beams are independently controlled with the micromirror device, the illumination duration for each beam or stimulation site can be tuned to produce subthreshold responses or action potential firing, independent from the number of stimulation sites and the stimulation frequency. This is a major advantage over single-beam systems, where illumination duration, the number of sites and the stimulation frequency variables are interdependent. Recently developed holographic photostimulation techniques share some advantages with parallel photostimulation $[78,79]$. However, they depend on a relatively slow light modulation technique (spatial light modulators) limiting the temporal precision to between 33 and $15 \mathrm{~ms}$. Current holographic systems are thus not capable of reproducing 
fast dynamic neuronal activity patterns. Their strength lies in the investigation of spatial integration through the ability to define uncaging locations in 3 dimensions.

Because the system described in this dissertation is based on computer-controlled DLP technology, it allows for convenient programming of photostimulation patterns using graphical user interfaces. Stimulation sites can be selected visually or preprogrammed patterns can be positioned to target specific cortical layers or the area of an individual dendrite. These unique features of parallel light stimulation make this technique well suited for the investigation of the complex spatiotemporal neuronal activity patterns and their functional significance for dendritic integration, synaptic plasticity and the propagation of activity through the neuronal network. A unique strength of the new system is that the degree of synchrony as well as the size and spatial distribution of neuronal populations can be easily manipulated to answer long-standing questions about the propagation of oscillatory and synchronous activity in a biological network of randomly firing neurons [53]. Experiments based mostly on intracellular current injections strongly suggest that synchronous synaptic input is coupled to precisely timed action potential initiation in both cortical [137,179] and hippocampal [169] pyramidal neurons. By stimulating in L4 and recording intracellular responses in L2/3, we demonstrated the use of DLP photostimulation to investigate how synchronous activity in one layer of cortex influences spiking activity in another layer. The membrane potentials of cortical neurons in vivo exhibit rapid voltage fluctuations caused by ongoing activity of thousands of synaptic inputs $[180,181]$. This background activity influences the physiological properties and action potential output of neurons[173,182,183]. With parallel light stimulation it is possible to independently control random background activity and synchronous activity through separate groups of stimulation sites. This provides new approaches for the investigation of the influence of ongoing background activity on the generation and propagation of synchronous spiking activity.

It should also be possible to combine DLP photostimulation with voltage sensitive dye, calcium indicator, or other activity indicator imaging, allowing investigations of the propagation of population activity in cortical slices as a function of the degree of synchrony and population size. This system could also be easily adapted to manipulate the activity of genetically defined subsets of neurons by changing the light source and photostimulating neural tissues expressing light-sensitive ion channels. The introduction of multiple stimulation wavelengths is technically straightforward and will allow the independent control of distinct groups of neurons expressing different light-sensitive ion channels gated by different wavelengths.

The system could also be adapted to manipulate neuronal activity in vivo by performing experiments in animals expressing light-sensitive ion channels. This can be accomplished with a fixed projection system similar to the one described here in head fixed or anesthetized preparations or via flexible fiber optic light guides for freely moving mice [184]. DLP based photostimulation opens up important new experimental opportunities to investigate the complex spatiotemporal neuronal interactions underlying the dynamic activity patterns that are characteristic for the conscious brain. 


\section{LIST OF REFERENCES}

1. Adrian E, Bronk DW, Phillips G (1932) Discharges in mammalian sympathetic nerves. The Journal of Physiology 74: 115.

2. Adrian ED, Bronk DW (1929) The discharge of impulses in motor nerve fibres: Part II. The frequency of discharge in reflex and voluntary contractions. J Physiol (Lond) 67: i3-151.

3. Adrian ED, Bronk DW (1928) The discharge of impulses in motor nerve fibres: Part I. Impulses in single fibres of the phrenic nerve. J Physiol (Lond) 66: 81-101.

4. Knight BW (1972) Dynamics of encoding in a population of neurons. The Journal of General Physiology 59: 734.

5. Softky WR, Koch C (1993) The highly irregular firing of cortical cells is inconsistent with temporal integration of random EPSPs. J Neurosci 13: 334-350.

6. Shadlen MN, Newsome WT (1998) The variable discharge of cortical neurons: implications for connectivity, computation, and information coding. The Journal of Neuroscience 18: 3870.

7. Riehle A (1997) Spike Synchronization and Rate Modulation Differentially Involved in Motor Cortical Function. Science 278: 1950-1953. doi:10.1126/science.278.5345.1950.

8. Aertsen A, Diesmann M, Gewaltig MO, Grün S, Rotter S (2001) Neural dynamics in cortical networks--precision of joint-spiking events. Novartis Found Symp 239: 193-204; discussion 204-207, 234-240.

9. Woolsey TA, Van der Loos H (1970) The structural organization of layer IV in the somatosensory region (SI) of mouse cerebral cortex. The description of a cortical field composed of discrete cytoarchitectonic units. Brain Res 17: 205242.

10. Simons DJ, Woolsey TA (1979) Functional organization in mouse barrel cortex. Brain Res 165: 327-332.

11. Simons DJ (1978) Response properties of vibrissa units in rat SI somatosensory neocortex. J Neurophysiol 41: 798-820.

12. Brecht M, Sakmann B (2002) Dynamic representation of whisker deflection by synaptic potentials in spiny stellate and pyramidal cells in the barrels and septa of layer 4 rat somatosensory cortex. J Physiol (Lond) 543: 49-70.

13. Agmon A, Connors BW (1991) Thalamocortical responses of mouse somatosensory (barrel) cortex in vitro. Neuroscience 41: 365-379.

14. Petersen CC, Sakmann B (2000) The excitatory neuronal network of rat layer 4 barrel cortex. J Neurosci 20: 7579-7586.

15. Petersen CCH, Sakmann B (2001) Functionally independent columns of rat somatosensory barrel cortex revealed with voltage-sensitive dye imaging. The journal of Neuroscience 21: 8435.

16. Moore CI, Nelson SB (1998) Spatio-temporal subthreshold receptive fields in the vibrissa representation of rat primary somatosensory cortex. J Neurophysiol 80: 2882-2892.

17. Zhu JJ, Connors BW (1999) Intrinsic firing patterns and whisker-evoked synaptic responses of neurons in the rat barrel cortex. J Neurophysiol 81: 1171-1183. 
18. Fox K (2008) Barrel Cortex. 1st ed. Cambridge University Press. 318 p.

19. Lee KJ, Woolsey TA (1975) A proportional relationship between peripheral innervation density and cortical neuron number in the somatosensory system of the mouse. Brain Res 99: 349-353.

20. Li X, Glazewski S, Lin X, Elde R, Fox K (1995) Effect of vibrissae deprivation on follicle innervation, neuropeptide synthesis in the trigeminal ganglion, and S1 barrel cortex plasticity. J Comp Neurol 357: 465-481.

doi:10.1002/cne.903570310.

21. Zucker E, Welker WI (1969) Coding of somatic sensory input by vibrissae neurons in the rat's trigeminal ganglion. Brain Research 12: 138-156.

doi:10.1016/0006-8993(69)90061-4.

22. Shoykhet M, Doherty D, Simons DJ (2000) Coding of deflection velocity and amplitude by whisker primary afferent neurons: implications for higher level processing. Somatosens Mot Res 17: 171-180.

23. Gibson JM, Welker WI (1983) Quantitative studies of stimulus coding in firstorder vibrissa afferents of rats. 2. Adaptation and coding of stimulus parameters. Somatosens Res 1: 95-117.

24. Gibson JM, Welker WI (1983) Quantitative studies of stimulus coding in firstorder vibrissa afferents of rats. 1. Receptive field properties and threshold distributions. Somatosens Res 1: 51-67.

25. Jacquin MF, Renehan WE, Rhoades RW, Panneton WM (1993) Morphology and topography of identified primary afferents in trigeminal subnuclei principalis and oralis. J Neurophysiol 70: 1911-1936.

26. Jacquin MF, McCasland JS, Henderson TA, Rhoades RW, Woolsey TA (1993) 2DG uptake patterns related to single vibrissae during exploratory behaviors in the hamster trigeminal system. J Comp Neurol 332: 38-58.

doi:10.1002/cne.903320104.

27. Jacquin MF, Golden J, Panneton WM (1988) Structure and function of barrel "precursor" cells in trigeminal nucleus principalis. Brain Res 471: 309-314.

28. Jacquin MF, Stennett RA, Renehan WE, Rhoades RW (1988) Structure-function relationships in the rat brainstem subnucleus interpolaris: II. Low and high threshold trigeminal primary afferents. J Comp Neurol 267: 107-130. doi:10.1002/cne.902670108.

29. Chiaia NL, Zhang S, Crissman RS, Rhoades RW (2000) Effects of neonatal axoplasmic transport attenuation on the response properties of vibrissae-sensitive neurons in the trigeminal principal sensory nucleus of the rat. Somatosens Mot Res 17: 273-283.

30. Minnery BS, Simons DJ (2003) Response properties of whisker-associated trigeminothalamic neurons in rat nucleus principalis. J Neurophysiol 89: 40-56. doi:10.1152/jn.00272.2002.

31. Minnery BS, Bruno RM, Simons DJ (2003) Response transformation and receptive-field synthesis in the lemniscal trigeminothalamic circuit. J Neurophysiol 90: 1556-1570. doi:10.1152/jn.00111.2003.

32. Shipley MT (1974) Response characteristics of single units in the rat's trigeminal nuclei to vibrissa displacements. J Neurophysiol 37: 73-90. 
33. Veinante P, Deschênes M (1999) Single- and Multi-Whisker Channels in the Ascending Projections from the Principal Trigeminal Nucleus in the Rat. J Neurosci 19: 5085-5095.

34. Timofeeva E, Lavallée P, Arsenault D, Deschênes M (2004) Synthesis of multiwhisker-receptive fields in subcortical stations of the vibrissa system. J Neurophysiol 91: 1510-1515. doi:10.1152/jn.01109.2003.

35. Lu SM, Lin RC (1993) Thalamic afferents of the rat barrel cortex: a light- and electron-microscopic study using Phaseolus vulgaris leucoagglutinin as an anterograde tracer. Somatosens Mot Res 10: 1-16.

36. Bruno RM (2006) Cortex Is Driven by Weak but Synchronously Active Thalamocortical Synapses. Science 312: 1622-1627. doi:10.1126/science. 1124593.

37. Wang H-P, Spencer D, Fellous J-M, Sejnowski TJ (2010) Synchrony of thalamocortical inputs maximizes cortical reliability. Science 328: 106-109. doi:10.1126/science. 1183108 .

38. Temereanca S, Simons DJ (2003) Local field potentials and the encoding of whisker deflections by population firing synchrony in thalamic barreloids. J Neurophysiol 89: 2137-2145. doi:10.1152/jn.00582.2002.

39. Armstrong-James M, Fox K, Das-Gupta A (1992) Flow of excitation within rat barrel cortex on striking a single vibrissa. Journal of neurophysiology 68: 1345 .

40. Douglas RJ, Martin KAC (2004) Neuronal circuits of the neocortex. Annual Review of Neuroscience 27: 419-451. doi:10.1146/annurev.neuro.27.070203.144152.

41. Abeles M (1991) Corticonics: Neural Circuits of the Cerebral Cortex. Cambridge University Press.

42. Shadlen MN, Newsome WT (1994) Noise, neural codes and cortical organization. Curr Opin Neurobiol 4: 569-579.

43. Lübke J, Roth A, Feldmeyer D, Sakmann B (2003) Morphometric analysis of the columnar innervation domain of neurons connecting layer 4 and layer $2 / 3$ of juvenile rat barrel cortex. Cereb Cortex 13: 1051-1063.

44. Hirata A, Castro-Alamancos MA (2008) Cortical Transformation of Wide-Field (Multiwhisker) Sensory Responses. J Neurophysiol 100: 358-370. doi:10.1152/jn.90538.2008.

45. Kelly MK, Carvell GE, Kodger JM, Simons DJ (1999) Sensory loss by selected whisker removal produces immediate disinhibition in the somatosensory cortex of behaving rats. J Neurosci 19: 9117-9125.

46. Simons DJ (1983) Multi-whisker stimulation and its effects on vibrissa units in rat SmI barrel cortex. Brain Res 276: 178-182.

47. Simons DJ (1985) Temporal and spatial integration in the rat SI vibrissa cortex. J Neurophysiol 54: 615-635.

48. Ghazanfar AA, Nicolelis MA (1997) Nonlinear processing of tactile information in the thalamocortical loop. J Neurophysiol 78: 506-510.

49. Mirabella G, Battiston S, Diamond ME (2001) Integration of multiple-whisker inputs in rat somatosensory cortex. Cereb Cortex 11: 164-170. 
50. Nowak LG, Bullier J (1998) Axons, but not cell bodies, are activated by electrical stimulation in cortical gray matter. Experimental Brain Research 118: 477-488. doi:10.1007/s002210050304.

51. Feldmeyer D, Llübke J, Silver RA, Sakmann B (2002) Synaptic connections between layer 4 spiny neurone-layer $2 / 3$ pyramidal cell pairs in juvenile rat barrel cortex: physiology and anatomy of interlaminar signalling within a cortical column. The Journal of physiology 538: 803-822.

52. Sarid L, Bruno R, Sakmann B, Segev I, Feldmeyer D (2007) Modeling a layer 4to-layer $2 / 3$ module of a single column in rat neocortex: interweaving in vitro and in vivo experimental observations. Proc Natl Acad Sci USA 104: 16353-16358. doi:10.1073/pnas.0707853104.

53. Diesmann M, Gewaltig MO, Aertsen A (1999) Stable propagation of synchronous spiking in cortical neural networks. Nature 402: 529-533. doi:10.1038/990101.

54. Gewaltig MO, Diesmann M, Aertsen A (2001) Propagation of cortical synfire activity: survival probability in single trials and stability in the mean. Neural Netw 14: 657-673.

55. Cāteau H, Fukai T (2001) Fokker-Planck approach to the pulse packet propagation in synfire chain. Neural Networks 14: 675-685.

56. Litvak V, Sompolinsky H, Segev I, Abeles M (2003) On the transmission of rate code in long feedforward networks with excitatory-inhibitory balance. J Neurosci 23: 3006-3015.

57. Moradi F (2004) Information coding and oscillatory activity in synfire neural networks with and without inhibitory coupling. Biol Cybern 91: 283-294. doi:10.1007/s00422-004-0499-x.

58. Brette R, Rudolph M, Carnevale T, Hines M, Beeman D, et al. (2007) Simulation of networks of spiking neurons: a review of tools and strategies. J Comput Neurosci 23: 349-398. doi:10.1007/s10827-007-0038-6.

59. Teramae J-N, Fukai T (2007) Local cortical circuit model inferred from powerlaw distributed neuronal avalanches. J Comput Neurosci 22: 301-312. doi:10.1007/s10827-006-0014-6.

60. Pazienti A, Maldonado PE, Diesmann M, Grün S (2008) Effectiveness of systematic spike dithering depends on the precision of cortical synchronization. Brain Res 1225: 39-46. doi:10.1016/j.brainres.2008.04.073.

61. Kumar A, Rotter S, Aertsen A (2010) Spiking activity propagation in neuronal networks: reconciling different perspectives on neural coding. Nat Rev Neurosci 11: 615-627. doi:10.1038/nrn2886.

62. Kumar A, Rotter S, Aertsen A (2008) Conditions for propagating synchronous spiking and asynchronous firing rates in a cortical network model. J Neurosci 28: 5268-5280. doi:10.1523/JNEUROSCI.2542-07.2008.

63. Kremkow J, Perrinet LU, Masson GS, Aertsen A (2010) Functional consequences of correlated excitatory and inhibitory conductances in cortical networks. Journal of Computational Neuroscience 28: 579-594. doi:10.1007/s10827-010-0240-9.

64. Kaplan JH, Forbush B 3rd, Hoffman JF (1978) Rapid photolytic release of adenosine 5'-triphosphate from a protected analogue: utilization by the Na:K pump of human red blood cell ghosts. Biochemistry 17: 1929-1935. 
65. Wilcox M, Viola R, Johnson K, Billington A, Carpenter B, et al. (1990) Synthesis of photolabile precursors of amino acid neurotransmitters. Journal of Organic Chemistry 55: 1585-1589.

66. Matsubara N, Billington AP, Hess GP (1992) How fast does an acetylcholine receptor channel open? Laser-pulse photolysis of an inactive precursor of carbamoylcholine in the microsecond time region with $\mathrm{BC} 3 \mathrm{H} 1$ cells. Biochemistry 31: 5507-5514.

67. Corrie JE, DeSantis A, Katayama Y, Khodakhah K, Messenger JB, et al. (1993) Postsynaptic activation at the squid giant synapse by photolytic release of Lglutamate from a "caged" L-glutamate. J Physiol (Lond) 465: 1-8.

68. Callaway EM, Katz LC (1993) Photostimulation using caged glutamate reveals functional circuitry in living brain slices. Proceedings of the National Academy of Sciences 90: 7661.

69. Farber IC, Grinvald A (1983) Identification of presynaptic neurons by laser photostimulation. Science 222: 1025-1027.

70. Ellis-Davies GCR (2008) Neurobiology with caged calcium. Chem Rev 108: 1603-1613. doi:10.1021/cr078210i.

71. Dalva MB, Katz LC (1994) Rearrangements of synaptic connections in visual cortex revealed by laser photostimulation. Science 265: 255-258.

72. Katz LC, Dalva MB (1994) Scanning laser photostimulation: a new approach for analyzing brain circuits. J Neurosci Methods 54: 205-218.

73. Shepherd GMG, Pologruto TA, Svoboda K (2003) Circuit analysis of experiencedependent plasticity in the developing rat barrel cortex. Neuron 38: 277-289.

74. Maier W, Schemm R, Grewer C, Laube B (2007) Disruption of Interdomain Interactions in the Glutamate Binding Pocket Affects Differentially Agonist Affinity and Efficacy of N-methyl-d-aspartate Receptor Activation. Journal of Biological Chemistry 282: 1863 -1872. doi:10.1074/jbc.M608156200.

75. Trigo FF, Corrie JET, Ogden D (2009) Laser photolysis of caged compounds at $405 \mathrm{~nm}$ : photochemical advantages, localisation, phototoxicity and methods for calibration. J Neurosci Methods 180: 9-21. doi:10.1016/j.jneumeth.2009.01.032.

76. Boucsein C, Nawrot M, Rotter S, Aertsen A, Heck D (2005) Controlling synaptic input patterns in vitro by dynamic photo stimulation. J Neurophysiol 94: 29482958. doi:10.1152/jn.00245.2005.

77. Shoham S, O’Connor DH, Sarkisov DV, Wang SS-H (2005) Rapid neurotransmitter uncaging in spatially defined patterns. Nat Methods 2: 837-843. doi:10.1038/nmeth793.

78. Golan L, Reutsky I, Farah N, Shoham S (2009) Design and characteristics of holographic neural photo-stimulation systems. Journal of Neural Engineering 6: 066004. doi:10.1088/1741-2560/6/6/066004.

79. Dal Maschio M, Difato F, Beltramo R, Blau A, Benfenati F, et al. (2010) Simultaneous two-photon imaging and photo-stimulation with structured light illumination. Opt Express 18: 18720-18731. doi:10.1364/OE.18.018720.

80. Zahid M, Vélez-Fort M, Papagiakoumou E, Ventalon C, Angulo MC, et al. (2010) Holographic Photolysis for Multiple Cell Stimulation in Mouse Hippocampal Slices. PLoS ONE 5: e9431. doi:10.1371/journal.pone.0009431. 
81. Suga T, Hirano M, Takayanagi M, Koshimoto H, Watanabe A (1998) Restricted photorelease of biologically active molecules near the plasma membrane.

Biochem Biophys Res Commun 253: 423-430. doi:10.1006/bbrc.1998.9153.

82. Volgraf M, Gorostiza P, Szobota S, Max R, Isacoff EY, et al. (2007) Reversibly caged glutamate: a photochromic agonist of ionotropic glutamate receptors. Journal of the American Chemical Society 129: 260-261.

83. Pettit DL, Wang SSH, Gee KR, Augustine GJ (1997) Chemical two-photon uncaging: a novel approach to mapping glutamate receptors. Neuron 19: 465-471.

84. Dalva MB, Weliky M, Katz LC (1997) Relationships between local synaptic connections and orientation domains in primary visual cortex. Neuron 19: 871880.

85. Wang SSH, Khiroug L, Augustine GJ (2000) Quantification of spread of cerebellar long-term depression with chemical two-photon uncaging of glutamate. Proceedings of the National Academy of Sciences 97: 8635.

86. Shembekar VR, Chen Y, Carpenter BK, Hess GP (2005) A Protecting Group for Carboxylic Acids That Can Be Photolyzed by Visible Light. Biochemistry 44: 7107-7114. doi:10.1021/bi047665o.

87. Fino E, Araya R, Peterka DS, Salierno M, Etchenique R, et al. (2009) RuBiglutamate: two-photon and visible-light photoactivation of neurons and dendritic spines. Frontiers in Neural Circuits 3.

88. Godwin DW, Che D, O'Malley DM, Zhou Q (1997) Photostimulation with caged neurotransmitters using fiber optic lightguides. Journal of Neuroscience Methods 73: 91-106. doi:10.1016/S0165-0270(96)02208-X.

89. Ghezzi D, Pedrocchi A, Menegon A, Mantero S, Valtorta F, et al. (2007) PhotoMEA: an opto-electronic biosensor for monitoring in vitro neuronal network activity. BioSystems 87: 150-155. doi:10.1016/j.biosystems.2006.09.008.

90. Ghezzi D, Menegon A, Pedrocchi A, Valtorta F, Ferrigno G (2008) A MicroElectrode Array device coupled to a laser-based system for the local stimulation of neurons by optical release of glutamate. J Neurosci Methods 175: 70-78. doi:10.1016/j.jneumeth.2008.08.003.

91. Kandler K, Katz LC, Kauer JA (1998) Focal photolysis of caged glutamate produces long-term depression of hippocampal glutamate receptors. Nat Neurosci 1: 119-123. doi:10.1038/368.

92. Aravanis AM, Wang L-P, Zhang F, Meltzer LA, Mogri MZ, et al. (2007) An optical neural interface: in vivo control of rodent motor cortex with integrated fiberoptic and optogenetic technology. Journal of Neural Engineering 4: S143S156. doi:10.1088/1741-2560/4/3/S02.

93. Campagnola L, Wang H, Zylka MJ (2008) Fiber-coupled light-emitting diode for localized photostimulation of neurons expressing channelrhodopsin-2. J Neurosci Methods 169: 27-33. doi:10.1016/j.jneumeth.2007.11.012.

94. Einstein A (1905) Über einen die Erzeugung und Verwandlung des Lichtes betreffenden heuristischen Gesichtspunkt. Annalen der Physik 322: 132-148.

95. Göppert-Mayer M (1931) Ueber Elementarakte mit zwei Quantensprüngen. Ann Phys 401: 273-294.

96. Abella I (1962) Optical Double-Photon Absorption in Cesium Vapor. Physical Review Letters 9: 453-455. 
97. Peticolas W, Goldsborough, Rieckhoff K (1963) Double photon excitation in organic crystals. Phys Rev Lett: 43-45.

98. Denk W, Strickler JH, Webb WW (1990) Two-photon laser scanning fluorescence microscopy. Science 248: 73.

99. Furuta T, Wang SSH, Dantzker JL, Dore TM, Bybee WJ, et al. (1999) Brominated 7-hydroxycoumarin-4-ylmethyls: photolabile protecting groups with biologically useful cross-sections for two photon photolysis. Proceedings of the National Academy of Sciences 96: 1193.

100. Matsuzaki M, Ellis-Davies GC, Nemoto T, Miyashita Y, Iino M, et al. (2001) Dendritic spine geometry is critical for AMPA receptor expression in hippocampal CA1 pyramidal neurons. Nat Neurosci 4: 1086-1092. doi:10.1038/nn736.

101. Papageorgiou G, Ogden DC, Barth A, Corrie JET (1999) Photorelease of Carboxylic Acids from 1-Acyl-7-nitroindolines in Aqueous Solution: Rapid and Efficient Photorelease of 1-Glutamate1. Journal of the American Chemical Society 121: 6503-6504. doi:10.1021/ja990931e.

102. Canepari M, Nelson L, Papageorgiou G, Corrie JE, Ogden D (2001) Photochemical and pharmacological evaluation of 7-nitroindolinyl-and 4methoxy-7-nitroindolinyl-amino acids as novel, fast caged neurotransmitters. $\mathbf{J}$ Neurosci Methods 112: 29-42.

103. Papageorgiou G, Corrie JE. (2000) Effects of Aromatic Substituents on the Photocleavage of 1-Acyl-7-nitroindolines. Tetrahedron 56: 8197-8205. doi:10.1016/S0040-4020(00)00745-6.

104. Fedoryak OD, Sul J-Y, Haydon PG, Ellis-Davies GCR (2005) Synthesis of a caged glutamate for efficient one- and two-photon photorelease on living cells. Chemical Communications: 3664. doi:10.1039/b504922a.

105. Nikolenko V, Poskanzer KE, Yuste R (2007) Two-photon photostimulation and imaging of neural circuits. Nature Methods 4: 943-950. doi:10.1038/nmeth1105.

106. Matsuzaki M, Ellis-Davies GCR, Kasai H (2008) Three-Dimensional Mapping of Unitary Synaptic Connections by Two-Photon Macro Photolysis of Caged Glutamate. Journal of Neurophysiology 99: 1535-1544. doi:10.1152/jn.01127.2007.

107. Nikolenko V, Watson BO, Araya R, Woodruff A, Peterka DS, et al. (2008) SLM microscopy: scanless two-photon imaging and photostimulation with spatial light modulators. Frontiers in Neural Circuits 2.

108. Kreiter AK, Singer W (1996) Stimulus-dependent synchronization of neuronal responses in the visual cortex of the awake macaque monkey. J Neurosci 16: 2381-2396.

109. Dan Y, Alonso JM, Usrey WM, Reid RC (1998) Coding of visual information by precisely correlated spikes in the lateral geniculate nucleus. Nat Neurosci 1: 501507. doi:10.1038/2217.

110. deCharms RC, Merzenich MM (1996) Primary cortical representation of sounds by the coordination of action-potential timing. Nature 381: 610-613. doi:10.1038/381610a0. 
111. Huetz C, Philibert B, Edeline J-M (2009) A spike-timing code for discriminating conspecific vocalizations in the thalamocortical system of anesthetized and awake guinea pigs. J Neurosci 29: 334-350. doi:10.1523/JNEUROSCI.3269-08.2009.

112. Gutierrez R, Simon SA, Nicolelis MAL (2010) Licking-induced synchrony in the taste-reward circuit improves cue discrimination during learning. J Neurosci 30: 287-303. doi:10.1523/JNEUROSCI.0855-09.2010.

113. Vaadia E, Haalman I, Abeles M, Bergman H, Prut Y, et al. (1995) Dynamics of neuronal interactions in monkey cortex in relation to behavioural events. Nature 373: 515-518. doi:10.1038/373515a0.

114. Steinmetz PN, Roy A, Fitzgerald PJ, Hsiao SS, Johnson KO, et al. (2000) Attention modulates synchronized neuronal firing in primate somatosensory cortex. Nature 404: 187-190. doi:10.1038/35004588.

115. Salinas E, Sejnowski TJ (2001) Correlated neuronal activity and the flow of neural information. Nat Rev Neurosci 2: 539-550. doi:10.1038/35086012.

116. Single-Panel DLP Projection System Optics (2005). Available:http://focus.ti.com/pdfs/dlpdmd/Discoverydlpa002.pdf.

117. Wang H, Peca J, Matsuzaki M, Matsuzaki K, Noguchi J, et al. (2007) High-speed mapping of synaptic connectivity using photostimulation in Channelrhodopsin-2 transgenic mice. Proceedings of the National Academy of Sciences 104: 8143.

118. Dhawale AK, Hagiwara A, Bhalla US, Murthy VN, Albeanu DF (2010) Nonredundant odor coding by sister mitral cells revealed by light addressable glomeruli in the mouse. Nat Neurosci 13: 1404-1412. doi:10.1038/nn.2673.

119. Leifer AM, Fang-Yen C, Gershow M, Alkema MJ, Samuel ADT (2011) Optogenetic manipulation of neural activity in freely moving Caenorhabditis elegans. Nat Meth 8: 147-152. doi:10.1038/nmeth.1554.

120. Liang CW, Mohammadi M, Santos MD, Santos MD, Tang C-M (2011) Patterned photostimulation with digital micromirror devices to investigate dendritic integration across branch points. J Vis Exp. Available:http://www.ncbi.nlm.nih.gov/pubmed/21403635. Accessed 15 September 2011.

121. Jerome J, Foehring RC, Armstrong WE, Spain WJ, Heck DH (2011) Parallel Optical Control of Spatiotemporal Neuronal Spike Activity Using High-Speed Digital Light Processing. Front Syst Neurosci 5. doi:10.3389/fnsys.2011.00070.

122. Losonczy A, Makara JK, Magee JC (2008) Compartmentalized dendritic plasticity and input feature storage in neurons. Nature 452: 436-441. doi:10.1038/nature06725.

123. Dantzker JL, Callaway EM (2000) Laminar sources of synaptic input to cortical inhibitory interneurons and pyramidal neurons. Nat Neurosci 3: 701-707. doi:10.1038/76656.

124. Feldmeyer D, Egger V, Lubke J, Sakmann B (1999) Reliable synaptic connections between pairs of excitatory layer 4 neurones within a single "barrel" of developing rat somatosensory cortex. J Physiol (Lond) 521 Pt 1: 169-190.

125. Woolsey TA, Van der Loos H (1970) The structural organization of layer IV in the somatosensory region (SI) of mouse cerebral cortex. The description of a cortical field composed of discrete cytoarchitectonic units. Brain Res 17: 205242. 
126. Schüz A, Palm G (1989) Density of neurons and synapses in the cerebral cortex of the mouse. J Comp Neurol 286: 442-455. doi:10.1002/cne.902860404.

127. Tsai PS, Kaufhold JP, Blinder P, Friedman B, Drew PJ, et al. (2009) Correlations of neuronal and microvascular densities in murine cortex revealed by direct counting and colocalization of nuclei and vessels. The Journal of Neuroscience 29: 14553.

128. Bannister AP, Thomson AM (2007) Dynamic properties of excitatory synaptic connections involving layer 4 pyramidal cells in adult rat and cat neocortex. Cereb Cortex 17: 2190-2203. doi:10.1093/cercor/bhl126.

129. Bureau I, Shepherd GMG, Svoboda K (2008) Circuit and plasticity defects in the developing somatosensory cortex of FMR1 knock-out mice. J Neurosci 28: 51785188. doi:10.1523/JNEUROSCI.1076-08.2008.

130. Hooks BM, Hires SA, Zhang Y-X, Huber D, Petreanu L, et al. (2011) Laminar analysis of excitatory local circuits in vibrissal motor and sensory cortical areas. PLoS Biol 9: e1000572. doi:10.1371/journal.pbio.1000572.

131. Lefort S, Tomm C, Floyd Sarria J-C, Petersen CCH (2009) The excitatory neuronal network of the $\mathrm{C} 2$ barrel column in mouse primary somatosensory cortex. Neuron 61: 301-316. doi:10.1016/j.neuron.2008.12.020.

132. Sato TR, Gray NW, Mainen ZF, Svoboda K (2007) The functional microarchitecture of the mouse barrel cortex. PLoS Biol 5: e189. doi:10.1371/journal.pbio.0050189.

133. Armstrong-James M, Fox K (1987) Spatiotemporal convergence and divergence in the rat S1 "barrel" cortex. J Comp Neurol 263: 265-281. doi:10.1002/cne.902630209.

134. Fox K, Glazewski S, Chen CM, Silva A, Li X (1996) Mechanisms underlying experience-dependent potentiation and depression of vibrissae responses in barrel cortex. J Physiol Paris 90: 263-269.

135. Stosiek C, Garaschuk O, Holthoff K, Konnerth A (2003) In Vivo Two-Photon Calcium Imaging of Neuronal Networks. PNAS 100: 7319-7324. doi:10.1073/pnas.1232232100.

136. Bruno RM, Sakmann B (2006) Cortex is driven by weak but synchronously active thalamocortical synapses. Science 312: 1622-1627. doi:10.1126/science.1124593.

137. Nettleton JS, Spain WJ (2000) Linear to supralinear summation of AMPAmediated EPSPs in neocortical pyramidal neurons. Journal of neurophysiology 83: 3310 .

138. Waters J, Larkum M, Sakmann B, Helmchen F (2003) Supralinear Ca2+ influx into dendritic tufts of layer $2 / 3$ neocortical pyramidal neurons in vitro and in vivo. J Neurosci 23: 8558-8567.

139. Larkum ME, Waters J, Sakmann B, Helmchen F (2007) Dendritic spikes in apical dendrites of neocortical layer $2 / 3$ pyramidal neurons. The Journal of Neuroscience 27: 8999.

140. Svoboda K, Helmchen F, Denk W, Tank DW (1999) Spread of dendritic excitation in layer $2 / 3$ pyramidal neurons in rat barrel cortex in vivo. Nat Neurosci 2: 65-73. doi:10.1038/4569. 
141. van Brederode JF, Foehring RC, Spain WJ (2000) Morphological and electrophysiological properties of atypically oriented layer 2 pyramidal cells of the juvenile rat neocortex. Neuroscience 101: 851-861.

142. Larkman AU (1991) Dendritic morphology of pyramidal neurones of the visual cortex of the rat: I. Branching patterns. J Comp Neurol 306: 307-319. doi:10.1002/cne.903060207.

143. Larkman AU, Major G, Stratford KJ, Jack JJ (1992) Dendritic morphology of pyramidal neurones of the visual cortex of the rat. IV: Electrical geometry. $\mathrm{J}$ Comp Neurol 323: 137-152. doi:10.1002/cne.903230202.

144. Zador AM, Agmon-Snir H, Segev I (1995) The morphoelectrotonic transform: a graphical approach to dendritic function. J Neurosci 15: 1669-1682.

145. Schaefer AT, Larkum ME, Sakmann B, Roth A (2003) Coincidence detection in pyramidal neurons is tuned by their dendritic branching pattern. J Neurophysiol 89: 3143-3154. doi:10.1152/jn.00046.2003.

146. Stein RB (1967) The information capacity of nerve cells using a frequency code. Biophys J 7: 797-826. doi:10.1016/S0006-3495(67)86623-2.

147. van Rossum MCW, Turrigiano GG, Nelson SB (2002) Fast propagation of firing rates through layered networks of noisy neurons. The Journal of neuroscience 22: 1956.

148. Gautrais J, Thorpe S (1998) Rate coding versus temporal order coding: a theoretical approach. BioSystems 48: 57-65.

149. Van Rullen R, Thorpe SJ (2001) Rate coding versus temporal order coding: what the retinal ganglion cells tell the visual cortex. Neural Comput 13: 1255-1283.

150. Knight BW (1972) The relationship between the firing rate of a single neuron and the level of activity in a population of neurons. Experimental evidence for resonant enhancement in the population response. J Gen Physiol 59: 767-778.

151. Schmolesky MT, Wang Y, Hanes DP, Thompson KG, Leutgeb S, et al. (1998) Signal timing across the macaque visual system. J Neurophysiol 79: 3272-3278.

152. Gray CM, König P, Engel AK, Singer W (1989) Oscillatory responses in cat visual cortex exhibit inter-columnar synchronization which reflects global stimulus properties. Nature 338: 334-337. doi:10.1038/338334a0.

153. Wilson HR, Cowan JD (1972) Excitatory and inhibitory interactions in localized populations of model neurons. Biophys J 12: 1-24. doi:10.1016/S00063495(72)86068-5.

154. van Vreeswijk C, Sompolinsky H (1998) Chaotic balanced state in a model of cortical circuits. Neural Comput 10: 1321-1371.

155. van Vreeswijk C, Sompolinsky H (1996) Chaos in neuronal networks with balanced excitatory and inhibitory activity. Science 274: 1724-1726.

156. Mar DJ, Chow CC, Gerstner W, Adams RW, Collins JJ (1999) Noise shaping in populations of coupled model neurons. Proc Natl Acad Sci USA 96: 1045010455.

157. Thorpe S, Fize D, Marlot C (1996) Speed of processing in the human visual system. Nature 381: 520-522. doi:10.1038/381520a0.

158. Kotowicz A, Rutishauser U, Koch C (2010) Time course of target recognition in visual search. Front Hum Neurosci 4: 31. doi:10.3389/fnhum.2010.00031. 
159. Potter MC (1976) Short-term conceptual memory for pictures. J Exp Psychol Hum Learn 2: 509-522.

160. Fabre-Thorpe M (2011) The characteristics and limits of rapid visual categorization. Front Psychol 2: 243. doi:10.3389/fpsyg.2011.00243.

161. Gray CM, Singer W (1989) Stimulus-specific neuronal oscillations in orientation columns of cat visual cortex. Proceedings of the National Academy of Sciences 86: 1698.

162. Gray CM, Engel AK, König P, Singer W (1992) Synchronization of oscillatory neuronal responses in cat striate cortex: temporal properties. Vis Neurosci 8: $337-$ 347.

163. Aertsen A, Vaadia E, Abeles M, Ahissar E, Bergman H, et al. (1991) Neural interactions in the frontal cortex of a behaving monkey: signs of dependence on stimulus context and behavioral state. J Hirnforsch 32: 735-743.

164. Abeles M (1982) Role of the cortical neuron: integrator or coincidence detector? Isr J Med Sci 18: 83-92.

165. Softky W (1994) Sub-millisecond coincidence detection in active dendritic trees. Neuroscience 58: 13-41.

166. König P, Engel AK, Singer W (1996) Integrator or coincidence detector? The role of the cortical neuron revisited. Trends Neurosci 19: 130-137.

167. Mel BW (1993) Synaptic integration in an excitable dendritic tree. J Neurophysiol 70: 1086-1101.

168. Margulis M, Tang CM (1998) Temporal integration can readily switch between sublinear and supralinear summation. J Neurophysiol 79: 2809-2813.

169. Gasparini S, Magee JC (2006) State-dependent dendritic computation in hippocampal CA1 pyramidal neurons. The Journal of neuroscience 26: 2088.

170. Gasparini S, Migliore M, Magee JC (2004) On the initiation and propagation of dendritic spikes in CA1 pyramidal neurons. J Neurosci 24: 11046-11056. doi:10.1523/JNEUROSCI.2520-04.2004.

171. Losonczy A, Magee JC (2006) Integrative properties of radial oblique dendrites in hippocampal CA1 pyramidal neurons. Neuron 50: 291-307.

172. Gerstner W (2000) Population dynamics of spiking neurons: fast transients, asynchronous states, and locking. Neural Comput 12: 43-89.

173. Fellous J-M, Rudolph M, Destexhe A, Sejnowski TJ (2003) Synaptic background noise controls the input/output characteristics of single cells in an in vitro model of in vivo activity. Neuroscience 122: 811-829.

174. Mehring C, Hehl U, Kubo M, Diesmann M, Aertsen A (2003) Activity dynamics and propagation of synchronous spiking in locally connected random networks. Biological Cybernetics 88: 395-408. doi:10.1007/s00422-002-0384-4.

175. Melzer P, Champney GC, Maguire MJ, Ebner FF (2006) Rate code and temporal code for frequency of whisker stimulation in rat primary and secondary somatic sensory cortex. Experimental Brain Research 172: 370-386. doi:10.1007/s00221005-0334-1.

176. Reyes AD (2003) Synchrony-dependent propagation of firing rate in iteratively constructed networks in vitro. Nat Neurosci 6: 593-599. doi:10.1038/nn1056.

177. Ranganathan GN, Koester HJ (2011) Correlations Decrease with Propagation of Spiking Activity in the Mouse Barrel Cortex. Frontiers in Neural Circuits 5. 
178. Aertsen A, Diesmann M, Gewaltig MO (1996) Propagation of synchronous spiking activity in feedforward neural networks. J Physiol Paris 90: 243-247.

179. Rodriguez-Molina VM, Aertsen A, Heck DH (2007) Spike timing and reliability in cortical pyramidal neurons: effects of EPSC kinetics, input synchronization and background noise on spike timing. PLoS ONE 2: e319.

doi:10.1371/journal.pone.0000319.

180. Steriade M, Timofeev I, Grenier F (2001) Natural waking and sleep states: a view from inside neocortical neurons. J Neurophysiol 85: 1969-1985.

181. Stern EA, Kincaid AE, Wilson CJ (1997) Spontaneous subthreshold membrane potential fluctuations and action potential variability of rat corticostriatal and striatal neurons in vivo. J Neurophysiol 77: 1697-1715.

182. Chance FS, Abbott LF, Reyes AD (2002) Gain modulation from background synaptic input. Neuron 35: 773-782.

183. Léger J-F, Stern EA, Aertsen A, Heck D (2005) Synaptic integration in rat frontal cortex shaped by network activity. J Neurophysiol 93: 281-293. doi:10.1152/jn.00067.2003.

184. Huber D, Petreanu L, Ghitani N, Ranade S, Hromádka T, et al. (2008) Sparse optical microstimulation in barrel cortex drives learned behaviour in freely moving mice. Nature 451: 61-64. doi:10.1038/nature06445. 


\section{Example Importation and Marshalling of ALP DLL Functions into C\#}

Importing and marshalling of ALP functions requires correct C\# equivalents of $\mathrm{C}++$ data types. The following commonly used functions, as written below, import correctly.

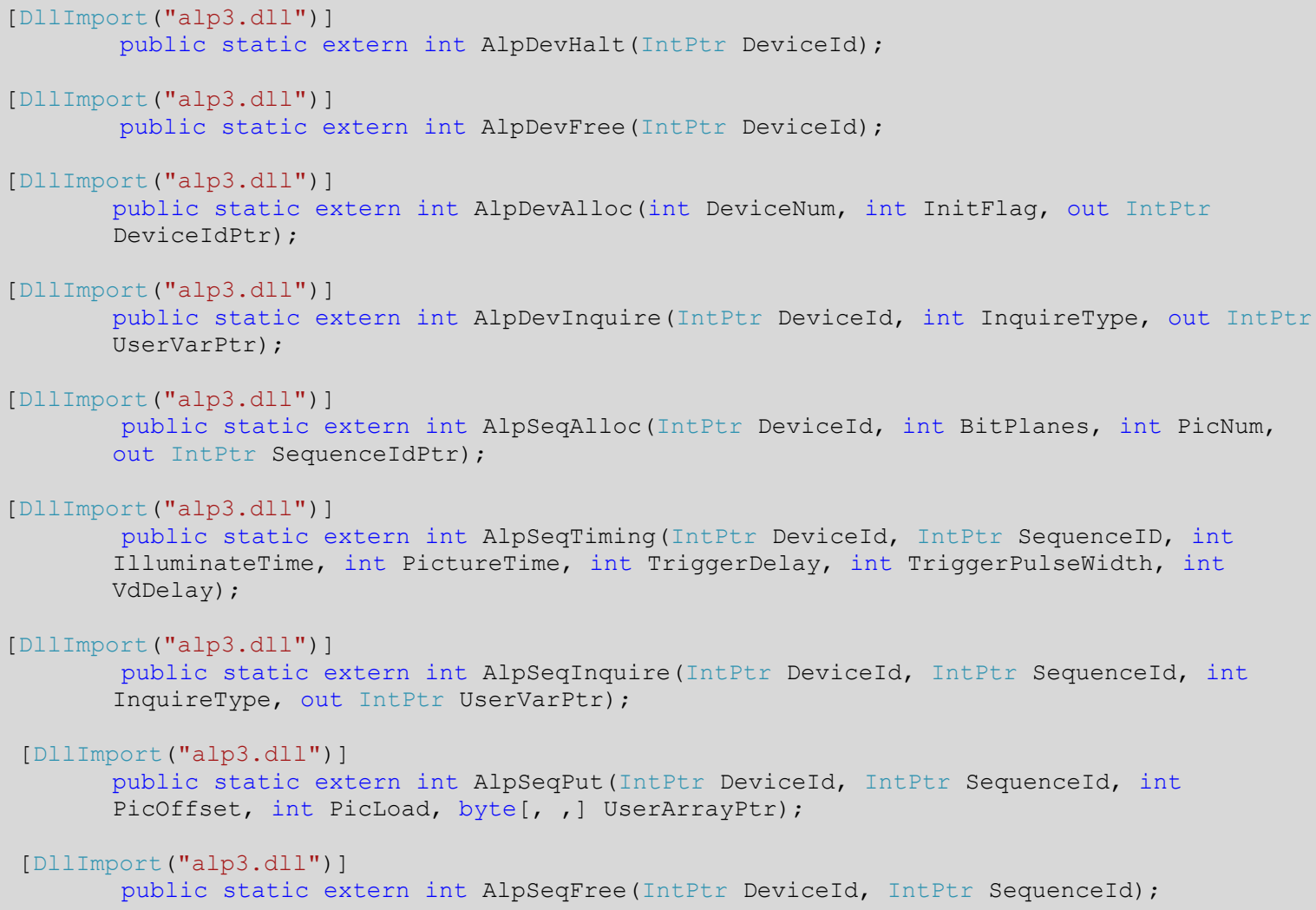




\section{Example Initilization of the DLP/ALP System}

The code below initializes USB communication with the DLP system and returns information on the device state.

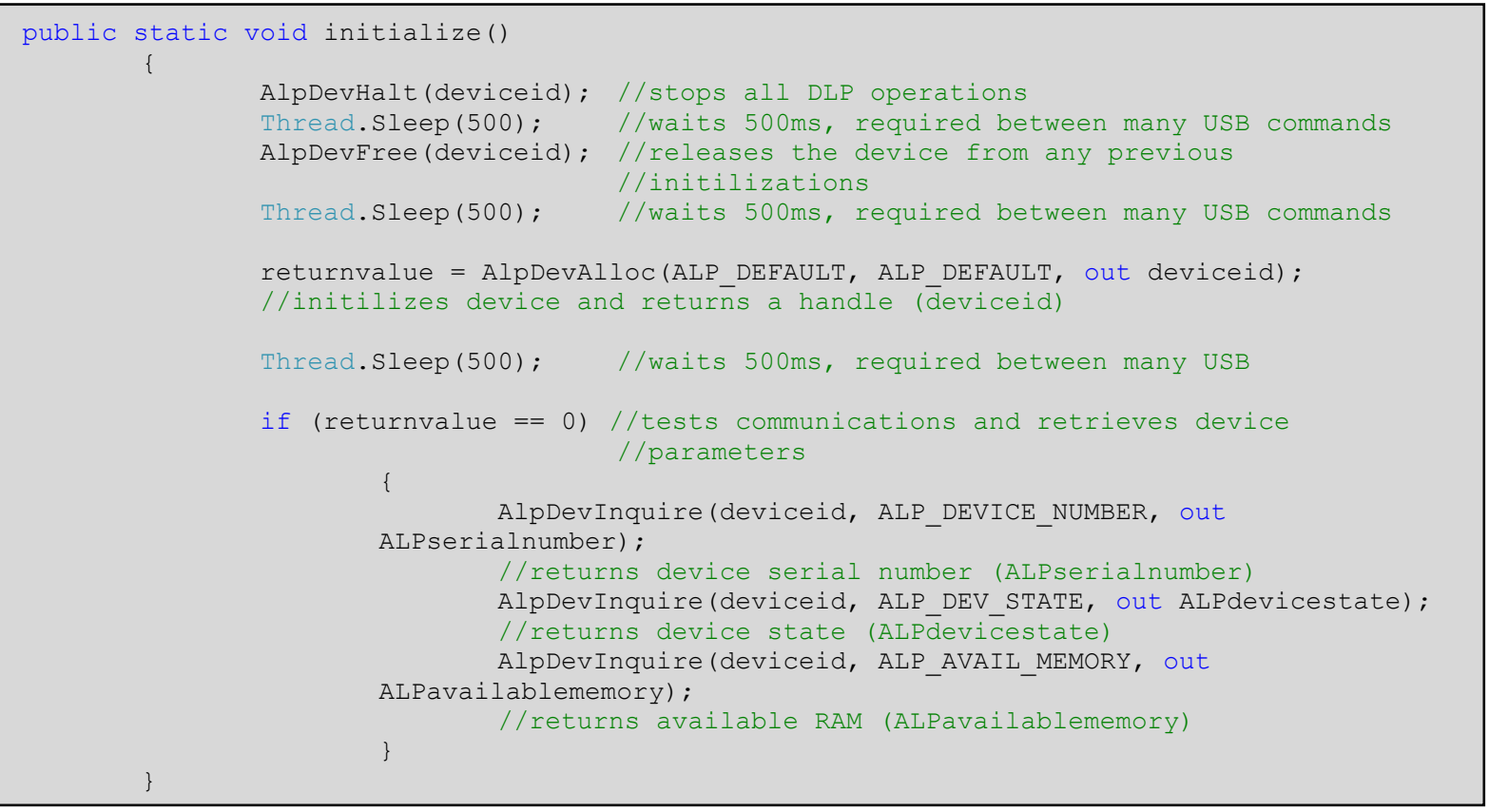




\section{Example of Loading a Sequence to ALP RAM}

The code below shows an example of using the graphics capabilities of $\mathrm{C \#}$ to generate a simple projection sequence, convert the sequence to ALP-3 friendly format, allocate RAM on the ALP-3, and load the sequence into allocated ALP-3 RAM.

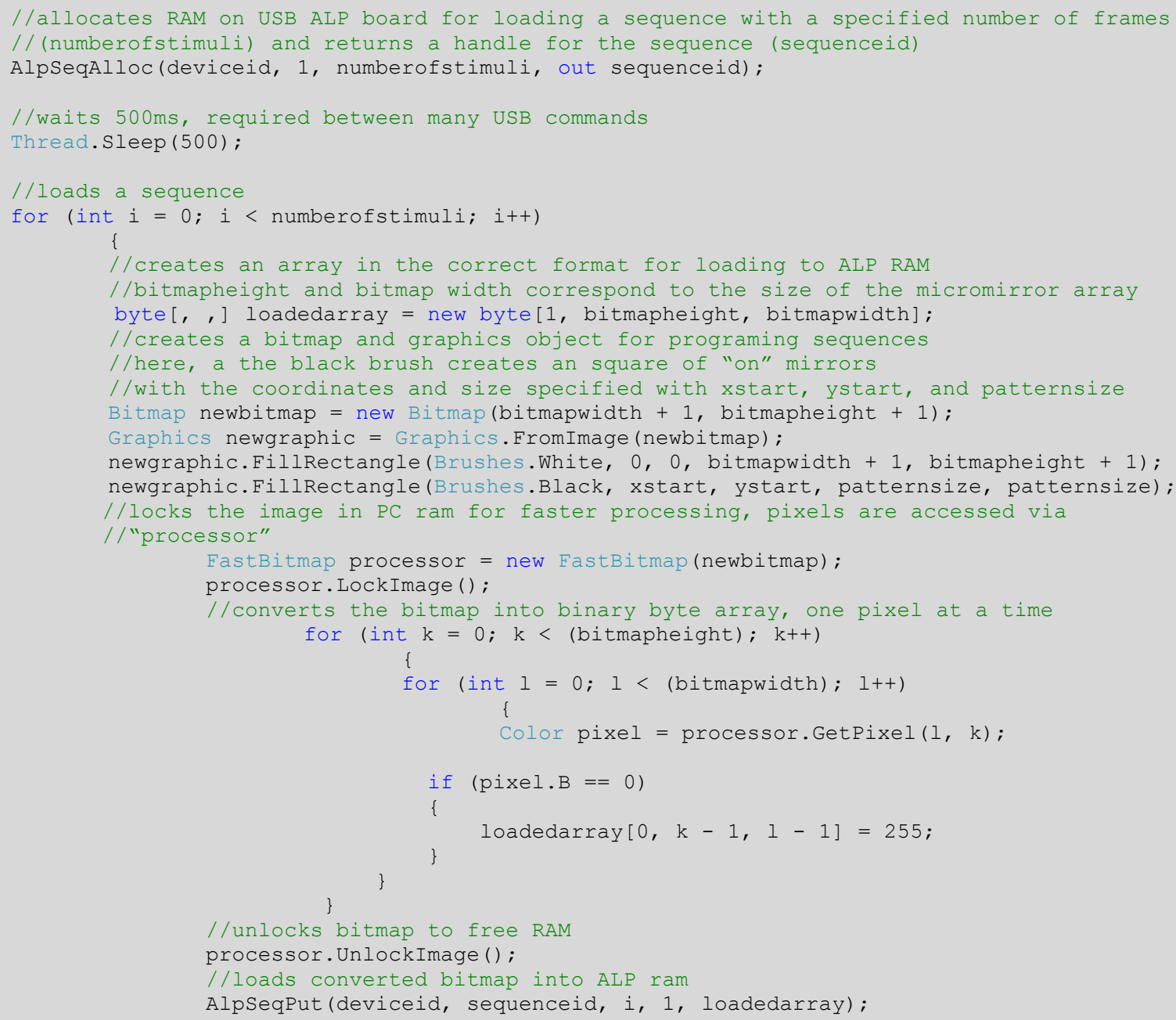




\section{Example of Starting and Stopping a Sequences with Button Clicks}

The following code shows how GUI buttons start and stop the projection of previously loaded sequences.

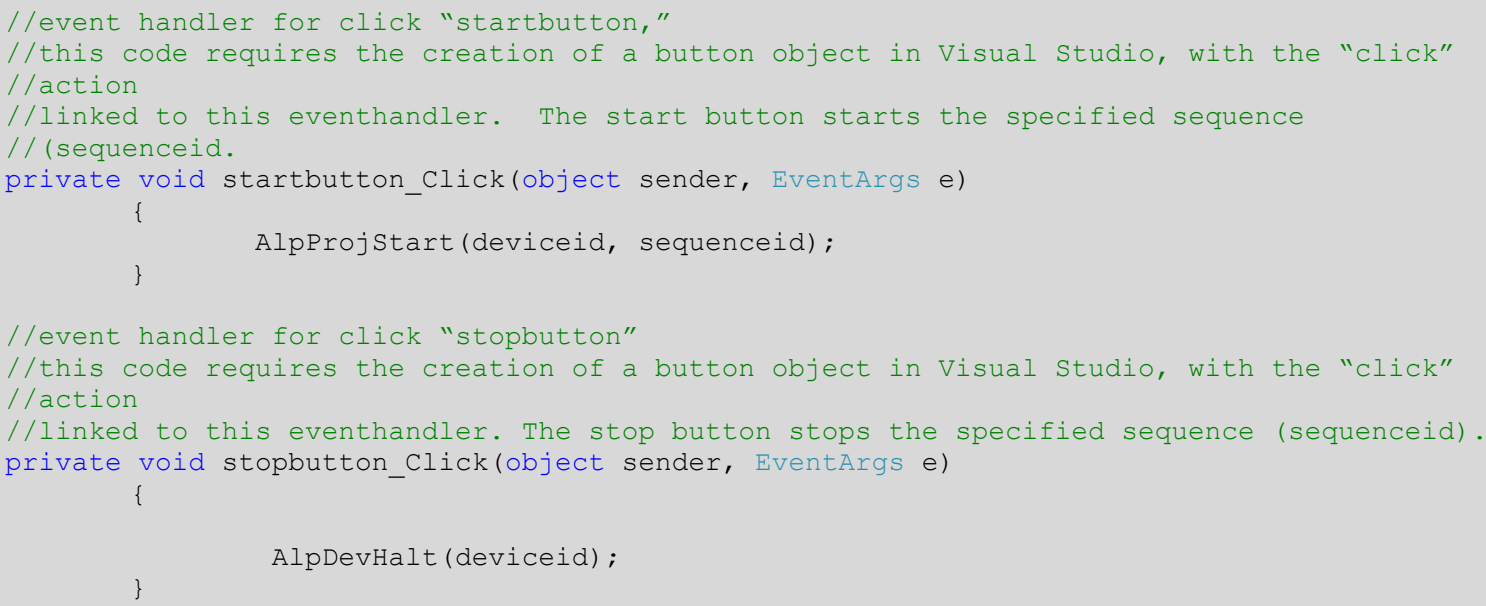




\section{VITA}

Jason Jerome was born in 1984 in Winston-Salem, North Carolina. In 2002, he graduated from South Whidbey High School, which is on an island in the Puget Sound in the state of Washington. His undergraduate studies were performed at The University of Alabama in Huntsville while working full-time at AZ Technology, a spaceflight technology company. He earned his B.S. in physics-engineering in December of 2006. He joined the Integrated Program in Biomedical Sciences (IPBS) at the University of Tennessee Health Science Center (UTHSC) in August 2007. 